\title{
The Cut-off Phenomenon for Brownian Motions on Compact Symmetric Spaces
}

\author{
Méliot, Pierre-Loïc
}

\begin{abstract}
In this paper, we prove the cut-off phenomenon in total variation distance for the Brownian motions traced on the classical symmetric spaces of compact type, that is to say: 1 . the classical simple compact Lie groups: special orthogonal groups $\mathrm{SO}(\mathrm{n})$, special unitary groups $\mathrm{SU}(\mathrm{n})$ and compact symplectic groups USp(n); 2. the real, complex and quaternionic Grassmannian varieties (including the real spheres, and the complex or quaternionic projective spaces when $q=1): S O(p+q) /(\operatorname{SO}(p) \times S O(q))$, $\mathrm{SU}(\mathrm{p}+\mathrm{q}) / \mathrm{S}(\mathrm{U}(\mathrm{p}) \times \mathrm{U}(\mathrm{q}))$ and $\mathrm{USp}(\mathrm{p}+\mathrm{q}) /(\mathrm{USp}(\mathrm{p}) \times \mathrm{USp}(\mathrm{q}))$; 3 . the spaces of real, complex and quaternionic structures: $\mathrm{SU}(\mathrm{n}) / \mathrm{SO}(\mathrm{n}), \mathrm{SO}(2 \mathrm{n}) / \mathrm{U}(\mathrm{n}), \mathrm{SU}(2 \mathrm{n}) / \mathrm{USp}(\mathrm{n})$ and $\mathrm{USp}(\mathrm{n}) / \mathrm{UU}(\mathrm{n})$. Denoting t the law of the Brownian motion at time $\mathrm{t}$, we give explicit lower bounds for $\mathrm{d} \mathrm{TV}(\mathrm{t}$, Haar $)$ if $t<t_{\text {cut-off }}=\alpha \log n$ , and explicit upper bounds if $t>t_{\text {cut-off }}$. This provides in particular an answer to some questions raised in recent papers by Chen and Saloff-Coste. Our proofs are inspired by those given by Rosenthal and Porod for products of random rotations in $\mathrm{SO}(\mathrm{n})$, and by Diaconis and Shahshahani for products of random transpositions in $\mathfrak{S}_{n}$.
\end{abstract}

DOI: https://doi.org/10.1007/s11118-013-9356-7

Posted at the Zurich Open Repository and Archive, University of Zurich

ZORA URL: https://doi.org/10.5167/uzh-171999

Journal Article

Published Version

Originally published at:

Méliot, Pierre-Loïc (2014). The Cut-off Phenomenon for Brownian Motions on Compact Symmetric Spaces. Potential Analysis, 40(4):427-509.

DOI: https://doi.org/10.1007/s11118-013-9356-7 


\title{
The Cut-off Phenomenon for Brownian Motions on Compact Symmetric Spaces
}

\author{
Pierre-Loïc Méliot
}

Received: 5 February 2013 / Accepted: 10 June 2013 / Published online: 23 June 2013

(C) Springer Science+Business Media Dordrecht 2013

\begin{abstract}
In this paper, we prove the cut-off phenomenon in total variation distance for the Brownian motions traced on the classical symmetric spaces of compact type, that is to say:
\end{abstract}

1. the classical simple compact Lie groups: special orthogonal groups $\mathrm{SO}(n)$, special unitary groups $\mathrm{SU}(n)$ and compact symplectic groups $\mathrm{USp}(n)$;

2. the real, complex and quaternionic Grassmannian varieties (including the real spheres, and the complex or quaternionic projective spaces when $q=1)$ : $\mathrm{SO}(p+$ $q) /(\mathrm{SO}(p) \times \mathrm{SO}(q)), \mathrm{SU}(p+q) / \mathrm{S}(\mathrm{U}(p) \times \mathrm{U}(q))$ and $\operatorname{USp}(p+q) /(\mathrm{USp}(p) \times$ $\mathrm{USp}(q))$

3. the spaces of real, complex and quaternionic structures: $\mathrm{SU}(n) / \mathrm{SO}(n), \mathrm{SO}(2 n)$ / $\mathrm{U}(n), \operatorname{SU}(2 n) / \mathrm{USp}(n)$ and $\mathrm{USp}(n) / \mathrm{U}(n)$.

Denoting $\mu_{t}$ the law of the Brownian motion at time $t$, we give explicit lower bounds for $d_{\mathrm{TV}}\left(\mu_{t}\right.$, Haar) if $t<t_{\text {cut-off }}=\alpha \log n$, and explicit upper bounds if $t>t_{\text {cut-off }}$. This provides in particular an answer to some questions raised in recent papers by Chen and Saloff-Coste. Our proofs are inspired by those given by Rosenthal and Porod for products of random rotations in $\mathrm{SO}(n)$, and by Diaconis and Shahshahani for products of random transpositions in $\mathfrak{S}_{n}$.

Keywords Brownian motion • Lie groups • Symmetric spaces • Cut-off phenomenon $\cdot$ Spherical functions

Mathematics Subject Classifications (2010) $58 \mathrm{~J} 65 \cdot 53 \mathrm{C} 35 \cdot 60 \mathrm{~B} 10 \cdot 60 \mathrm{~B} 15 \cdot 43 \mathrm{~A} 90$ 


\section{Introduction}

\subsection{The Cut-off Phenomenon for Random Permutations}

This paper is concerned with the analogue for Brownian motions on compact Lie groups and symmetric spaces of the famous cut-off phenomenon observed in random shuffles of cards (cf. $[1,4])$. Let us recall this result in the case of "natural" shuffles of cards, also known as riffle shuffles. Consider a deck of $n$ ordered cards $1,2, \ldots, n$, originally in this order. At each time $k \geq 1$, one performs the following procedure:

1. One cuts the deck in two parts of sizes $m$ and $n-m$, the integer $m$ being chosen randomly according to a binomial law of parameter $\frac{1}{2}$ :

$$
\mathbb{P}[m=M]=\frac{1}{2^{n}}\left(\begin{array}{c}
n \\
M
\end{array}\right) .
$$

So for instance, if $n=10$ and the deck was initially $123456789 \mathrm{X}$, then one obtains the two blocks $A=123456$ and $B=789 \mathrm{X}$ with probability $\frac{1}{2^{10}}\left(\begin{array}{c}10 \\ 6\end{array}\right)=\frac{105}{512} \simeq 0.21$.

2. The first card of the new deck comes from $A$ with probability (card $A$ ) $/ n$, and from $B$ with probability ( $\operatorname{card} B) / n$. Then, if $A^{\prime}$ and $B^{\prime}$ are the remaining blocks after removal of the first card, the second card of the new deck will come from $A^{\prime}$ with probability (card $\left.A^{\prime}\right) /(n-1)$, and from $B^{\prime}$ with probability (card $\left.B^{\prime}\right) /(n-$ 1); and similarly for the other cards. So for instance, by shuffling $A=123456$ and $B=789$ X, one can obtain with probability $1 /\left(\begin{array}{c}10 \\ 6\end{array}\right) \simeq 0.0048$ the deck 17283459 X6 .

Denote $\mathfrak{S}_{n}$ the symmetric group of order $n$, and $\sigma^{(k)}$ the random permutation in $\mathfrak{S}_{n}$ obtained after $k$ independent shuffles. One can guess that as $k$ goes to infinity, the law $\mathbb{P}^{(k)}$ of $\sigma^{(k)}$ converges to the uniform law $\mathbb{U}$ on $\mathfrak{S}_{n}$.

There is a natural distance on the set $\mathscr{P}\left(\mathfrak{S}_{n}\right)$ of probability measures on $\mathfrak{S}_{n}$ that allows to measure this convergence: the so-called total variation distance $d_{\mathrm{TV}}$. Consider more generally a measurable space $X$ with $\sigma$-field $\mathcal{B}(X)$. The total variation distance is the metric on the set of probability measures $\mathscr{P}(X)$ defined by

$$
d_{\mathrm{TV}}(\mu, v)=\sup \{|\mu(A)-v(A)|, \quad A \in \mathcal{B}(X)\} \in[0,1] .
$$

The convergence in total variation distance is in general a stronger notion than the weak convergence of probability measures. On the other hand, if $\mu$ and $v$ are absolutely continuous with respect to a third measure $d x$ on $X$, then their total variation distance can be written as a $\mathscr{L}^{1}$-norm:

$$
d_{\mathrm{TV}}(\mu, v)=\frac{1}{2} \int_{X}\left|\frac{d \mu}{d x}(x)-\frac{d v}{d x}(x)\right| d x .
$$

It turns out that with respect to total variation distance, the convergence of random shuffles occurs at a specific time $k_{\text {cut-off }}$, that is to say that $d_{\mathrm{TV}}\left(\mathbb{P}^{(k)}, \mathbb{U}\right)$ stays close to 1 for $k<k_{\text {cut-off }}$, and that $d_{\mathrm{TV}}\left(\mathbb{P}^{(k)}, \mathbb{U}\right)$ is then extremely close to 0 for

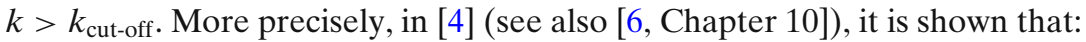

Theorem 1 (Bayer-Diaconis) Assume $k=\frac{3}{2 \log 2} \log n+\theta$. Then,

$$
d_{\mathrm{TV}}\left(\mathbb{P}^{(k)}, \mathbb{U}\right)=1-2 \phi\left(\frac{-2^{-\theta}}{4 \sqrt{3}}\right)+O\left(n^{-1 / 4}\right), \quad \text { with } \phi(x)=\frac{1}{\sqrt{2 \pi}} \int_{-\infty}^{x} \mathrm{e}^{-\frac{s^{2}}{2}} d s .
$$


So for $\theta$ negative, the total variation distance is extremely close to 1 , whereas it is extremely close to 0 for $\theta$ positive.

The cut-off phenomenon has been proved for other shuffling algorithms (e.g. random transpositions of cards), and more generally for large classes of finite Markov chains, see for instance [7, 9, 26]. It has also been investigated by Chen and SaloffCoste for Markov processes on continuous spaces, e.g. spheres and Lie groups; see in particular $[5,25,27]$ and the discussion of Section 1.4. However, in this case, cut-offs are easier to prove for the $\mathscr{L}^{p>1}$-norm of $p_{t}(x)-1$, where $p_{t}(x)$ is the density of the process at time $t$ and point $x$ with respect to the equilibrium measure. The case of the $\mathscr{L}^{1}$-norm, which is (up to a factor 2) the total variation distance, is somewhat different. In particular, a proof of the cut-off phenomenon for the total variation distance between the Haar measure and the marginal law $\mu_{t}$ of the Brownian motion on a classical compact Lie group was until now not known-see the remark just after [5, Theorem 1.2], and also [28, Conjecture 2]. The purpose of this paper is precisely to give a proof of this $\mathscr{L}^{1}$-cut-off for all classical compact Lie groups, and more generally for all classical symmetric spaces of compact type. In the two next paragraphs, we describe the spaces in which we will be interested (Section 1.2), and we precise what is meant by "Brownian motion" on a space of this type (cf. Section 1.3). This will then enable us to explain the results of Chen and Saloff-Coste in Section 1.4, and finally to state in Section 1.5 which improvements we were able to prove.

\subsection{Classical Compact Lie Groups and Symmetric Spaces}

To begin with, let us fix some notations regarding the three classical families of simple compact Lie groups, and their quotients corresponding to irreducible simply connected compact symmetric spaces. We use here most of the conventions of $[14,15]$. For every $n \geq 1$, we denote $\mathrm{U}(n)=\mathrm{U}(n, \mathbb{C})$ the unitary group of order $n ; \mathrm{O}(n)=$ $\mathrm{O}(n, \mathbb{R})$ the orthogonal group of order $n$; and $\operatorname{USp}(n)=\operatorname{USp}(n, \mathbb{H})$ the compact symplectic group of order $n$. They are defined by the same equations:

$$
U U^{\dagger}=U^{\dagger} U=I_{n} \quad ; \quad O O^{t}=O^{t} O=I_{n} \quad ; \quad S S^{\star}=S^{\star} S=I_{n}
$$

with complex, real or quaternionic coefficients, the conjugate of a quaternion $w+$ $\mathrm{i} x+\mathrm{j} y+\mathrm{k} z$ being $w-\mathrm{i} x-\mathrm{j} y-\mathrm{k} z$. The orthogonal groups are not connected, so we shall rather work with the special orthogonal groups

$$
\mathrm{SO}(n)=\mathrm{SO}(n, \mathbb{R})=\{O \in \mathrm{O}(n, \mathbb{R}) \mid \operatorname{det} O=1\} .
$$

On the other hand, the unitary groups are not simple Lie groups (their center is onedimensional), so it is convenient to introduce the special unitary groups

$$
\mathrm{SU}(n)=\mathrm{SU}(n, \mathbb{C})=\{U \in \mathrm{U}(n, \mathbb{C}) \mid \operatorname{det} U=1\} .
$$


Then, for every $n \geq 1, \mathrm{SU}(n, \mathbb{C}), \mathrm{SO}(n, \mathbb{R})$ and $\operatorname{USp}(n, \mathbb{H})$ are connected simple compact real Lie groups, of respective dimensions

$\operatorname{dim}_{\mathbb{R}} \operatorname{SU}(n, \mathbb{C})=n^{2}-1 \quad ; \quad \operatorname{dim}_{\mathbb{R}} \operatorname{SO}(n, \mathbb{R})=\frac{n(n-1)}{2} \quad ; \quad \operatorname{dim}_{\mathbb{R}} \mathrm{USp}(n, \mathbb{H})=2 n^{2}+n$

The special unitary groups and compact symplectic groups are simply connected; on the other hand, for $n \geq 3$, the fundamental group of $\operatorname{SO}(n, \mathbb{R})$ is $\mathbb{Z} / 2 \mathbb{Z}$, and its universal cover is the spin group $\operatorname{Spin}(n)$.

Many computations on these simple compact Lie groups can be performed by using their representation theory, which is covered by the highest weight theorem; see Section 2.2. We shall recall all this briefly in Section 2, and give in each case the list of all irreducible representations, and the corresponding dimensions and characters. It is well known that every simply connected compact simple Lie group is:

- $\quad$ either one group in the infinite families $\operatorname{SU}(n), \operatorname{Spin}(n), \operatorname{USp}(n)$;

- or, an exceptional simple compact Lie group of type $\mathrm{E}_{6}, \mathrm{E}_{7}, \mathrm{E}_{8}, \mathrm{~F}_{4}$ or $\mathrm{G}_{2}$.

We shall refer to the first case as the classical simple compact Lie groups, and as mentioned before, our goal is to study Brownian motions on these groups.

We shall more generally be interested in compact symmetric spaces; see e.g [14, Chapter 4]. These spaces can be defined by a local condition on geodesics, and by Cartan-Ambrose-Hicks theorem, a symmetric space $X$ is isomorphic as a Riemannian manifold to $G / K$, where $G$ is the connected component of the identity in the isometry group of $X$; $K$ is the stabilizer of a point $x \in X$ and a compact subgroup of $G$; and $(G, K)$ is a symmetric pair, which means that $K$ is included in the group of fixed points $G^{\theta}$ of an involutive automorphism of $G$, and contains the connected component $\left(G^{\theta}\right)^{0}$ of the identity in this group. Moreover, $X$ is compact if and only if $G$ is compact. This result reduces the classification of symmetric spaces to the classification of real Lie groups and their involutive automorphisms. So, consider an irreducible simply connected symmetric space, of compact type. Two cases arise:

1. The isometry group $G=K \times K$ is the product of a compact simple Lie group with itself, and $K$ is embedded into $G$ via the diagonal map $k \mapsto(k, k)$. The symmetric space $X$ is then the group $K$ itself, the quotient map from $G$ to $X \simeq K$ being

$$
\begin{aligned}
G & \rightarrow K \\
g=\left(k_{1}, k_{2}\right) & \mapsto k_{1} k_{2}^{-1} .
\end{aligned}
$$

In particular, the isometries of $K$ are the multiplication on the left and the right by elements of $K \times K$, and this action restricted to $K \subset G$ is the action by conjugacy.

2. The isometry group $G$ is a compact simple Lie group, and $K$ is a closed subgroup of it. In this case, there exists in fact a non-compact simple Lie group $L$ with maximal compact subgroup $K$, such that $G$ is a compact subgroup of the complexified Lie group $L^{\mathbb{C}}$, and maximal among those containing $K$. The involutive automorphism $\theta$ extends to $L^{\mathbb{C}}$, with $K=G^{\theta}=L^{\theta}$ and the two orthogonal symmetric Lie algebras $\left(\mathfrak{g}, d_{e} \theta\right)$ and $\left(\mathfrak{l}, d_{e} \theta\right)$ dual of each other. 
The classification of irreducible simply connected compact symmetric spaces is therefore the following: in addition to the compact simple Lie groups themselves, there are the seven infinite families

$$
\begin{aligned}
& \mathrm{Gr}(p+q, q, \mathbb{R})=\mathrm{SO}(p+q) /(\mathrm{SO}(p) \times \mathrm{SO}(q)) \\
& \text { with } p, q \geq 1 \text { (real Grassmannians); } \\
& \mathrm{Gr}(p+q, q, \mathbb{C})=\mathrm{SU}(p+q) / \mathrm{S}(\mathrm{U}(p) \times \mathrm{U}(q)) \\
& \text { with } p, q \geq 1 \text { (complex Grassmannians); } \\
& \operatorname{Gr}(p+q, q, \mathbb{H})=\operatorname{USp}(p+q) /(\operatorname{USp}(p) \times \operatorname{USp}(q)) \\
& \text { with } p, q \geq 1 \text { (quaternionic Grassmannians); }
\end{aligned}
$$

and quotients involving exceptional Lie groups, e.g. $\mathbb{P}^{2}(\mathbb{O})=\mathrm{F}_{4} / \operatorname{Spin}(9)$; see $[14$, Chapter 10]. For the two last families, one sees $\mathrm{U}(n)$ as a subgroup of $\mathrm{SO}(2 n)$ by replacing each complex number $x+\mathrm{i} y$ by the $2 \times 2$ real matrix

$$
\left(\begin{array}{cc}
x & y \\
-y & x
\end{array}\right)
$$

and one sees $\mathrm{USp}(n)$ as a subgroup of $\mathrm{SU}(2 n)$ by replacing each quaternion number $w+\mathrm{i} x+\mathrm{j} y+\mathrm{k} z$ by the $2 \times 2$ complex matrix

$$
\left(\begin{array}{cc}
w+\mathrm{i} x & y+\mathrm{i} z \\
-y+\mathrm{i} z & w-\mathrm{i} x
\end{array}\right)
$$

$\mathrm{USp}(n, \mathbb{H})$ is then the intersection of $\mathrm{SU}(2 n, \mathbb{C})$ and of the complex symplectic group $\operatorname{Sp}(2 n, \mathbb{C})$. We shall refer to the seven aforementioned families as classical simple compact symmetric spaces (of type non-group); again, we aim to study in detail the Brownian motions on these spaces.

\subsection{Laplace-Beltrami Operators and Brownian Motions on Symmetric Spaces}

We denote $d \eta_{K}(k)$ or $d k$ the Haar measure of a (simple) compact Lie group $K$, and $d \eta_{X}(x)$ or $d x$ the Haar measure of a compact symmetric space $X=G / K$, which is the image measure of $d \eta_{G}$ by the projection map $\pi: G \rightarrow G / K$. We refer to [15, Chapter 1] for precisions on the integration theory over (compact) Lie groups and their homogeneous spaces. There are several complementary ways to define a Brownian motion on a compact Lie group $K$ or a on compact symmetric space $G / K$, see in particular [20]. Hence, one can view them:

1. as Markov processes with infinitesimal generator the Laplace-Beltrami differential operator of the underlying Riemannian manifold;

2. as conjugacy-invariant continuous Lévy processes on $K$, or as projections of such a process on $G / K$; 
3. at least in the group case, as solutions of stochastic differential equations driven by standard (multidimensional) Brownian motions on the Lie algebra.

The first and the third point of view will be specially useful for our computations. For the sake of completeness, let us recall briefly each point of view-the reader already acquainted with these notions can go directly to Section 1.4.

\subsubsection{Choice of Normalization and Laplace-Beltrami Operators}

To begin with, let us precise the Riemannian structures chosen in each case. In the case of a simple compact Lie group $K$, the opposite of the Killing form $B(X, Y)=$ $\operatorname{tr}(\operatorname{ad} X \circ \operatorname{ad} Y)$ is negative-definite and gives by transport on each tangent space the unique bi- $K$-invariant Riemannian structure on $K$, up to a positive scalar. We choose this normalization constant as follows. When $K=\mathrm{SU}(n)$ or $\mathrm{SO}(n)$ or $\mathrm{USp}(n)$, the Killing form on $\mathfrak{k}$ is a scalar multiple of the bilinear form $X \otimes Y \mapsto \Re(\operatorname{tr}(X Y))$ - the real part is only needed for the quaternionic case. Then, we shall always consider the following invariant scalar products on $\mathfrak{k}$ :

$$
\langle X \mid Y\rangle=-\frac{\beta n}{2} \Re(\operatorname{tr}(X Y)),
$$

with $\beta=1$ for special orthogonal groups, $\beta=2$ for special unitary groups and unitary groups, and $\beta=4$ for compact symplectic groups (these are the conventions of e.g. [18]). Similarly, on a simple compact symmetric space $X=G / K$ of type nongroup, we take the previously chosen $\operatorname{Ad}(G)$-invariant scalar product (the one given by Eq. 1.3), and we restrict it to the orthogonal complement $\mathfrak{x}$ of $\mathfrak{k}$ in $\mathfrak{g}$. This $\mathfrak{x}$ can be identified with the tangent space of $X=G / K$ at $e K$, and by transport one gets the unique (up to a scalar) $G$-invariant Riemannian structure on $X$, called the Riemannian structure induced by the Riemannian structure of $G$. From now on, each classical simple compact symmetric space $X=G / K$ will be endowed with this induced Riemannian structure.

Remark This is not necessarily the "usual" normalization for these quotients: in particular, when $G=\mathrm{SO}(n+1)$ and $K=\mathrm{SO}(n) \times \mathrm{SO}(1)=\mathrm{SO}(n)$, the Riemannian structure defined by the previous conventions on the $n$-dimensional sphere $X=$ $\mathbb{S}^{n}(\mathbb{R})$ differs from the restriction of the standard euclidian metric of $\mathbb{R}^{n+1}$ by a factor $\sqrt{n+1}$. However this normalization does not change the nature of the cutoff phenomenon that we are going to prove.

Remark The bilinear form in Eq. 1.3 is only proportional to minus the Killing form, and not equal to it; for instance, the Killing form of $\mathrm{SO}(n, \mathbb{R})$ is

$$
(n-2) \operatorname{tr}(X Y)=-\frac{2 n-4}{n}\langle X \mid Y\rangle,
$$

and not $-\langle X \mid Y\rangle$. This leads to a factor 2 between our mixing times and those of $[5,25,27,28]$. However, the normalization of formula 1.3 enables one to relate the Brownian motions on the compact Lie groups to the "standard" Brownian motions on their Lie algebras, and to the classical ensembles of random matrix theory (see the SDEs at the end of this paragraph). 
The Laplace-Beltrami operator on a Riemannian manifold $M$ is the differential operator of degree 2 defined by

$$
\Delta f(m)=\sum_{1 \leq i, j \leq d} g^{i j}\left(\nabla_{X_{i}} \nabla_{X_{j}} f(m)-\nabla_{\nabla_{X_{i}} X_{j}} f(m)\right),
$$

where $\left(X_{1}, \ldots, X_{d}\right)$ is a basis of $T_{m} M,\left(g^{i j}\right)_{i, j}$ is the inverse of the metric tensor $\left(g_{i j}=\left\langle X_{i} \mid X_{j}\right\rangle_{T_{m} M}\right)_{i, j}$, and $\nabla_{X} Y$ denotes the covariant derivative of a vector $Y$ along a vector $X$ and with respect to the Levi-Civita connection. In the case of a compact Lie group $K$, this expression can be greatly simplified as follows (see for instance [20, Section 2.3]). Fix once and for all an orthonormal basis $\left(X_{1}, X_{2}, \ldots, X_{d}\right)$ of $\mathfrak{k}$. On another tangent space $T_{k} K$, one transports each $X_{i}$ by setting

$$
X_{i}^{l}(k)=\left\{d_{e} R_{k}\right\}\left(X_{i}\right) \in T_{k} K,
$$

where $R_{k}$ is the multiplication on the right by $k$. One thus obtains a vector field $X_{i}^{l}=\frac{\partial}{\partial x_{i}}$ which is left-invariant by construction and right-invariant because of the $\operatorname{Ad}(K)$-invariance of the scalar product on $\mathfrak{k}$. Then,

$$
\Delta=\sum_{i=1}^{d} \frac{\partial^{2}}{\partial x_{i}^{2}}
$$

Definition 2 A (standard) Brownian motion on a compact Riemannian manifold $M$ is a continuous Feller process $\left(m_{t}\right)_{t \in \mathbb{R}_{+}}$whose infinitesimal generator restricted to $\mathscr{C}^{2}(M)$ is $\frac{1}{2} \Delta$.

In the following, on a compact Lie group $K$ or a compact symmetric space $G / K$, we shall also assume that $m_{0}=e$ or $m_{0}=e K$ almost surely. We shall then denote $\mu_{t}$ the marginal law of the process at time $t$, and $p_{t}^{K}(k)=\frac{d \mu_{t}}{d \eta_{K}}(k)$ or $p_{t}^{X}(x)=\frac{d \mu_{t}}{d \eta_{X}}(x)$ the density of $\mu_{t}$ with respect to the Haar measure. General results about hypoelliptic diffusions on manifolds ensure that these densities exist for $t>0$ and are continuous in time and space; we shall later give explicit formulas for them (cf. Section 2).

\subsubsection{Brownian Motions as Continuous Lévy Processes}

By using the geometry of the spaces considered and the language of Lévy processes, one can give another equivalent definition of Brownian motions. The right increments of a random process $\left(g_{t}\right)_{t \in \mathbb{R}_{+}}$with values in a (compact) Lie group $G$ are the random variables $r_{t}^{s}=g_{s}^{-1} g_{t}$, so $g_{t}=g_{s} r_{t}^{s}$ for any times $s \leq t$. Then, a left Lévy process on $G$ is a càdlàg random process such that:

1. For any times $0=t_{0} \leq t_{1} \leq \cdots \leq t_{n}$, the right increments $r_{t_{1}}^{t_{0}}, r_{t_{2}}^{t_{1}}, \ldots, r_{t_{n}}^{t_{n-1}}$ are independent.

2. For any times $s \leq t$, the law of $r_{t}^{s}$ only depends on the difference $t-s: r_{t-s}^{0} \stackrel{\text { law }}{=} r_{t}^{s}$.

Denote $P_{t}$ the operator on the space $\mathscr{C}(G)$ of continuous functions on $G$ defined by $\left(P_{t} f\right)(g)=\mathbb{E}\left[f\left(g g_{t}\right)\right]$; and $\mu_{t}$ the law of $g_{t}$ assuming that $g_{0}=e_{G}$ almost surely. For $h \in G$, we also denote by $L_{h}$ the operator on $\mathscr{C}(G)$ defined by $L_{h} f(g)=f(h g)$. If $\left(g_{t}\right)_{t \in \mathbb{R}_{+}}$is a left Lévy process on $G$ starting at $g_{0}=e_{G}$, then:

1. The family of operators $\left(P_{t}\right)_{t \in \mathbb{R}_{+}}$is a Feller semigroup that is left $G$-invariant, meaning that $P_{t} \circ L_{h}=L_{h} \circ P_{t}$ for all $h \in G$ and for all time $t$. Conversely, any 
such Feller semigroup is the group of transitions of a left Lévy process which is unique in law.

2. The family of laws $\left(\mu_{t}\right)_{t \in \mathbb{R}_{+}}$is a semigroup of probability measures for the convolution product of measures

$$
(\mu * v)(f)=\int_{G^{2}} f(g h) d \mu(g) d v(h) .
$$

Hence, $\mu_{s} * \mu_{t}=\mu_{s+t}$ for any $s$ and $t$. Moreover, this semigroup is continuous, i.e., the limit in law $\lim _{t \rightarrow 0} \mu_{t}$ exists and is the Dirac measure $\delta_{e}$. Conversely, given such a semigroup of measures, there is always a corresponding left Lévy process, and it is unique in law.

Thus, left Lévy processes are the same as left $G$-invariant Feller semigroups of operators, and they are also the same as continuous semigroups of probability measures on $G$. In particular, on a compact Lie group, they are characterized by their infinitesimal generator

$$
L f(g)=\lim _{t \rightarrow \infty} \frac{P_{t} f(g)-f(g)}{t}
$$

defined on a suitable subspace of $\mathscr{C}(G)$. Hunt's theorem (cf. [17]) then characterizes the possible infinitesimal generators of (left) Lévy processes on a Lie group; in particular, continuous left-Lévy processes correspond to left-invariant differential operator of degree 2 .

Assume then that $\left(g_{t}\right)_{t \in \mathbb{R}_{+}}$is a continuous Lévy process on a simple compact Lie group $G$, starting from $e$ and with the additional property that $\left(h g_{t} h^{-1}\right)_{t \in \mathbb{R}_{+}}$and $\left(g_{t}\right)_{t \in \mathbb{R}_{+}}$have the same law in $\mathscr{C}\left(\mathbb{R}_{+}, G\right)$ for every $h$. These hypotheses imply that the infinitesimal generator $L$, which is a differential operator of degree 2 , is a scalar multiple of the Laplace-Beltrami operator $\Delta$. Thus, on a simple compact Lie group $K$, up to a linear change of time $t \mapsto$ at, a conjugacy-invariant continuous left Lévy process is a Brownian motion in the sense of Definition 2. Similarly, on a simple compact symmetric space $G / K$, up to a linear change of time, the image $\left(g_{t} K\right)_{t \in \mathbb{R}_{+}}$ of a conjugacy-invariant continuous left Lévy process on $G$ is a Brownian motion in the sense of Definition 2. This second definition of Brownian motions on compact symmetric spaces has the following important consequence:

Lemma 3 Let $\mu_{t}$ be the law of a Brownian motion on a compact Lie group $K$ or on a compact symmetric space $G / K$. The total variation distance $d_{\mathrm{TV}}\left(\mu_{t}\right.$, Haar) is a nonincreasing function of $t$.

Proof First, let us treat the case of compact Lie groups. If $f_{1}, f_{2}$ are in $\mathscr{L}^{1}\left(K, d \eta_{K}\right)$, then their convolution product $f_{1} * f_{2}$ is again in $\mathscr{L}^{1}(K)$, with

$$
\left\|f_{1} * f_{2}\right\|_{\mathscr{L}^{1}(K)} \leq\left\|f_{1}\right\|_{\mathscr{L}^{1}(K)}\left\|f_{2}\right\|_{\mathscr{L}^{1}(K)} .
$$

Now, since $\mu_{s+t}=\mu_{s} * \mu_{t}$, the densities of the Brownian motion also satisfy $p_{s+t}^{K}=$ $p_{s}^{K} * p_{t}^{K}$. Consequently,

$$
\begin{aligned}
2 d_{\mathrm{TV}}\left(\mu_{s+t}, \eta_{K}\right) & =\left\|p_{s+t}^{K}-1\right\|_{\mathscr{L}^{1}(K)}=\left\|\left(p_{s}^{K}-1\right) * p_{t}^{K}\right\|_{\mathscr{L}^{1}(K)} \\
& \leq\left\|p_{s}^{K}-1\right\|_{\mathscr{L}^{1}(K)}\left\|p_{t}^{K}\right\|_{\mathscr{L}^{1}(K)}=\left\|p_{s}^{K}-1\right\| \mathscr{L}^{1}(G)=2 d_{\mathrm{TV}}\left(\mu_{s}, \eta_{K}\right) .
\end{aligned}
$$


The proof is thus done in the group case. For a compact symmetric space $X=G / K$, denote $p_{t}^{G}$ the density of the Brownian motion on $G$, and $p_{t}^{X}$ the density of the Brownian motion on $X$. Since the Brownian motion on $X$ is the image of the Brownian motion on $G$ by $\pi: G \rightarrow G / K$, one has:

$$
\forall x=g K, \quad p_{t}^{X}(x)=\int_{K} p_{t}^{G}(g k) d k .
$$

As a consequence,

$$
\begin{aligned}
\left\|p_{s+t}^{X}-1\right\|_{\mathscr{L}^{1}(X)} & =\int_{G}\left|p_{s+t}^{X}(g K)-1\right| d g=\int_{G}\left|\int_{K}\left(p_{s+t}^{G}(g k)-1\right) d k\right| d g \\
& =\int_{G}\left|\int_{K \times G}\left(p_{s}^{G}\left(h^{-1} g k\right)-1\right) p_{t}^{G}(h) d k d h\right| d g \\
& =\int_{G}\left|\int_{G}\left(p_{s}^{X}\left(h^{-1} g K\right)-1\right) p_{t}^{G}(h) d h\right| d g \\
& \left.\leq \int_{G \times G} \mid p_{s}^{X}\left(h^{-1} g K\right)-1\right)|| p_{t}^{G}(h) \mid d h d g \\
& =\left\|p_{s}^{X}-1\right\|_{\mathscr{L}^{1}(X)}\left\|p_{t}^{G}\right\|_{\mathscr{L}^{1}(G)}=\left\|p_{s}^{X}-1\right\| \mathscr{L}^{1}(X),
\end{aligned}
$$

so $d_{\mathrm{TV}}\left(\mu_{s+t}, \eta_{X}\right) \leq d_{\mathrm{TV}}\left(\mu_{s}, \eta_{X}\right)$ also in the case of symmetric spaces.

Remark Later, this property will allow us to compute estimates of $d_{\mathrm{TV}}\left(\mu_{t}, \eta_{X}\right)$ only for $t$ around the cut-off time. Indeed, if one has for instance an (exponentially small) estimate of $1-d_{\mathrm{TV}}\left(\mu_{t_{0}}, \eta_{X}\right)$ at time $t_{0}=(1-\varepsilon) t_{\text {cut-off }}$, then the same estimate will also hold for $1-d_{\mathrm{TV}}\left(\mu_{t}, \eta_{X}\right)$ with $t<t_{0}$.

Remark Actually, the same result holds for the $\mathscr{L}^{p}$-norm of $p_{t}(x)-1$, and in the broader setting of Markov processes with a stationary measure; see e.g. [5, Proposition 3.1]. Our proof is a little more elementary.

\subsubsection{Brownian Motions as Solutions of SDE}

A third equivalent definition of Brownian motions on compact Lie groups is by mean of stochastic differential equations. More precisely, given a Brownian motion $\left(k_{t}\right)_{t \in \mathbb{R}_{+}}$ traced on a compact Lie group $K$, there exists a (trajectorially unique) standard $d$ dimensional Brownian motion $\left(W_{t}\right)_{t \in \mathbb{R}_{+}}$on the Lie algebra $\mathfrak{k}$ that drives stochastic differential equations for every test function $f \in \mathscr{C}^{2}(K)$ of $\left(k_{t}\right)_{t \in \mathbb{R}_{+}}$(cf. [20]). So for instance, on a unitary group $\mathrm{U}(n, \mathbb{C})$, the Brownian motion is the solution of the SDE

$$
U_{0}=I_{n} \quad ; \quad d U_{t}=\mathrm{i} U_{t} \cdot d H_{t}-\frac{1}{2} U_{t} d t
$$

where $\left(H_{t}\right)_{t \in \mathbb{R}_{+}}$is a Brownian hermitian matrix normalized so that at time $t=1$ the diagonal coefficients are independent real gaussian variables of variance $1 / n$, and the upper-diagonal coefficients are independent complex gaussian variables with real 
and imaginary parts independent and of variance $1 / 2 n$. In the general case, let us introduce the Casimir operator

$$
C=\sum_{i=1}^{d} X_{i} \otimes X_{i}
$$

This tensor should be considered as an element of the universal enveloping algebra $U(\mathfrak{k})$. Then, for every representation $\pi: K \rightarrow \mathrm{GL}(V)$, the image of $C$ by the infinitesimal representation $d \pi: U(\mathfrak{k}) \rightarrow \operatorname{End}(V)$ commutes with $d \pi(\mathfrak{k})$. In particular, for an irreducible representation $V, d \pi(C)$ is a scalar multiple $\kappa_{V} \mathrm{id}_{V}$ of $\mathrm{id}_{V}$. Assume that $K$ is a classical simple Lie group. Then its "geometric" representation is irreducible, so $\sum_{i=1}^{d}\left(X_{i}\right)^{2}=\alpha_{\mathfrak{k}} I_{n}$ if one sees the $X_{i}$ 's as matrices in $\mathrm{M}(n, \mathbb{R})$ or $\mathrm{M}(n, \mathbb{C})$ or $\mathrm{M}(n, \mathbb{H})$. The stochastic differential equation satisfied by a Brownian motion on $K$ is then

$$
k_{0}=e_{K} \quad ; \quad d k_{t}=k_{t} \cdot d B_{t}+\frac{\alpha_{\mathfrak{k}}}{2} k_{t} d t,
$$

where $B_{t}=\sum_{i=1}^{d} W_{t}^{i} X_{i}$ is a standard Brownian motion on the Lie algebra $\mathfrak{k}$. The constant $\alpha_{\mathfrak{k}}$ is given in the classical cases by

$$
\alpha_{\mathfrak{s u}(n)}=-\frac{n^{2}-1}{n^{2}} \quad ; \quad \alpha_{\mathfrak{s o}(n)}=-\frac{n-1}{n} \quad ; \quad \alpha_{\mathfrak{s p}(n)}=-\frac{2 n+1}{2 n}
$$

see [18, Lemma 1.2]. These Casimir operators will play a prominent role in the computation of the densities of these Brownian motions (cf. Section 2.2), and also at the end of this paper (Section 4.1), see Lemma 23.

\subsection{Chen-Saloff-Coste Results on $\mathscr{L}^{p}$-Cut-Offs of Markov Processes}

Fix $p \in[1, \infty)$, and consider a Markov process $\mathfrak{X}=\left(x_{t}\right)_{t \in \mathbb{R}_{+}}$with values in a measurable space $(X, \mathcal{B}(X))$, and admitting an invariant probability $\eta$. One denotes $\mu_{t, x}$ the marginal law of $x_{t}$ assuming $x_{0}=x$ almost surely, and

$$
d_{t}^{p}(\mathfrak{X})=\max _{x \in X}\left(\int_{X}\left|\frac{d \mu_{t, x}}{d \eta}(y)-1\right|^{p} \eta(d y)\right)^{\frac{1}{p}},
$$

with by convention

$$
d_{t}^{p}(\mathfrak{X})= \begin{cases}2 & \text { if } p=1, \\ +\infty & \text { if } p>1,\end{cases}
$$

when $\mu_{t, x}$ is not absolutely continuous with respect to $\eta$. This is obviously a generalization of the total variation distance to the stationary measure. In virtue of the remark stated just after Lemma $3, t \mapsto d_{t}^{p}(\mathfrak{X})$ is always non-increasing. A sequence of Markov processes $\left(\mathfrak{X}^{(n)}\right)_{n \in \mathbb{N}}$ with values in measurable spaces $\left(X^{(n)}, \mathcal{B}\left(X^{(n)}\right)\right)_{n \in \mathbb{N}}$ is said to have a max- $\mathscr{L}^{p}$-cut-off with cut-off times (or mixing times, or times to equilibrium) $\left(t^{(n)}\right)_{n \in \mathbb{N}}$ if

$\lim _{n \rightarrow \infty}\left(\sup _{t>(1+\varepsilon) t^{(n)}} d_{t}^{p}\left(\mathfrak{X}^{(n)}\right)\right)=0 \quad ; \quad \lim _{n \rightarrow \infty}\left(\inf _{t<(1-\varepsilon) t^{(n)}} d_{t}^{p}\left(\mathfrak{X}^{(n)}\right)\right)=\limsup _{n \rightarrow \infty} d_{0}^{p}\left(\mathfrak{X}^{(n)}\right)=M>0$ 
for every $\varepsilon \in(0,1)$ - usually $M$ will be equal to 2 or $+\infty$. A generalization of Theorem 1 ensures that these $\mathscr{L}^{p}$-cut-offs occur for instance in the case of riffle shuffles of cards, with $t^{(n)}=\frac{3 \log n}{2 \log 2}$ for every $p \in[1,+\infty$ ). We refer to [26] (in particular Section 2.4) for a detailed analysis of this notion in the case of finite Markov chains, and for the connections with the notions of spectral gap or hypercontractivity, and with the logarithmic Sobolev and the Nash inequalities.

In [5, Theorems 3.3 and 4.2], Chen and Saloff-Coste shown that a general criterion due to Peres ensures a $\mathscr{L}^{p>1}$-cut-off for a sequence of reversible Markov processes; but then one does not know necessarily the value of the mixing time $t^{(n)}$. Call spectral gap $\lambda(\mathfrak{X})$ of a Markov process $\mathfrak{X}$ the largest $c \geq 0$ such that for all $f \in \mathscr{L}^{2}(X, \eta)$ and all time $t,\left\|\left(P_{t}-\eta\right) f\right\|_{\mathscr{L}^{2}(X)} \leq \mathrm{e}^{-t c}\|f\|_{\mathscr{L}^{2}(X)}$, where $\left(P_{t}\right)_{t \in \mathbb{R}_{+}}$stands for the semigroup associated to the Markov process.

Theorem 4 (Chen-Saloff-Coste) Fix $p \in(1, \infty)$. One considers a family of Markov processes $\left(\mathfrak{X}^{(n)}\right)_{n \in \mathbb{N}}$ with self-adjoint operators $P_{t}$ and spectral gaps $\lambda^{(n)}$, and one assumes that $\lim _{t \rightarrow \infty} d_{t}^{p}\left(\mathfrak{X}^{(n)}\right)=0$ for every $n$. For $\varepsilon_{0}>0$ fixed, set

$$
t^{(n)}=\inf \left\{t: d_{t}^{p}\left(\mathfrak{X}^{(n)}\right) \leq \varepsilon_{0}\right\} .
$$

The family of Markov processes has a max- $\mathscr{L}^{p}$-cut-off if and only if Peres' criterion is satisfied:

$$
\lim _{n \rightarrow \infty} \lambda^{(n)} t^{(n)}=+\infty
$$

In this case, the sequence $\left(t^{(n)}\right)_{n \in \mathbb{N}}$ gives the values of the cut-off times. A lower bound on $t^{(n)}$ also ensures the cut-off phenomenon; but then, the mixing time remains unknown. Nevertheless, an important application of this general criterion is (see [5, Theorem 1.2]):

Corollary 5 (Saloff-Coste) Consider the Brownian motions traced on the special orthogonal groups $\mathrm{SO}(n, \mathbb{R})$, with the normalization of the metric detailed in the previous paragraph. They exhibit for every $p \in(1, \infty)$ a cut-off with $t^{(n)}$ asymptotically between $2 \log n$ and $4 \log n-$ notice that $t^{(n)}$ depends a priori on $p$.

Indeed, the spectral gap stays bounded and has a known non-negative limit (which we shall compute later, see the table on page 32$)$, whereas for any $p \in[1,+\infty), t^{(n)}$ was shown in [25, Theorem 1.2] and [27, Theorems 1.1 and 1.2] to be a $O(\log n)$, and more precisely

- $\quad$ in the window $[(2-o(1)) \log n,(4+o(1)) \log n]$ for simple compact Lie groups;

- in the window $[(1-o(1)) \log n,(2+o(1)) \log n]$ for simple compact symmetric spaces.

The proofs of these estimates were relying on precise logarithmic Sobolev inequalities and arguments of hypercontractivity for the simple compact Lie groups. The lower bound in the case of compact Lie groups was also known based on simple arguments of representation theory (cf. [25, Section 7]), that we shall use and generalize at the end of this paper (see Section 4). In [28, Conjecture 2], it is also conjectured 
that the correct value of the mixing time for $p \in[1,+\infty)$ is the lower bound of Corollary 5 , that is to say that for all simple compact Lie groups,

$$
t^{(n)} \simeq 2 \log n .
$$

The main result of our paper is a proof of this conjecture, for every classical simple compact Lie group or classical simple compact symmetric space, and with a cut-off time equal to $2 \log n$ or to $\log n$ depending on the type of the space considered.

\subsection{Statement of the Main Results and Discriminating Events}

Theorem 6 Let $\mu_{t}$ be the marginal law of the Brownian motion traced on a classical simple compact Lie group, or on classical simple compact symmetric space. There exists positive constants $\alpha, \gamma_{b}, \gamma_{a}, c, C$ and an integer $n_{0}$ such that in each family, for all $n \geq n_{0}$,

$$
\begin{aligned}
& \forall \varepsilon \in(0,1 / 4), \quad d_{\mathrm{TV}}\left(\mu_{t}, \text { Haar }\right) \geq 1-\frac{c}{n^{\gamma_{b} \varepsilon}} \quad \text { if } t=\alpha(1-\varepsilon) \log n ; \\
& \forall \varepsilon \in(0, \infty), \quad d_{\mathrm{TV}}\left(\mu_{t}, \text { Haar }\right) \leq \frac{C}{n^{\gamma_{a} \varepsilon / 4}} \quad \text { if } t=\alpha(1+\varepsilon) \log n .
\end{aligned}
$$

The constants $\alpha, \gamma_{b}$ and $\gamma_{a}$ are determined by the type of the space considered, and then one can make the following choices for $n_{0}, c$ and $C$ :

\begin{tabular}{lllllrrr}
\hline Kor $G / K$ & $\beta$ & $\alpha$ & $\gamma_{b}$ & $\gamma_{a}$ & $n_{0}$ & $c$ & $C$ \\
\hline $\mathrm{SO}(n, \mathbb{R})$ & 1 & 2 & 2 & 2 & 10 & 36 & 6 \\
$\mathrm{SU}(n, \mathbb{C})$ & 2 & 2 & 2 & 4 & 2 & 8 & 10 \\
$\mathrm{USp}(n, \mathbb{H})$ & 4 & 2 & 2 & 2 & 3 & 5 & 3 \\
$\operatorname{Gr}(n, q, \mathbb{R})$ & 1 & 1 & 1 & 1 & 10 & 32 & 2 \\
$\mathrm{Gr}(n, q, \mathbb{C})$ & 2 & 1 & 1 & 2 & 2 & 32 & 2 \\
$\mathrm{Gr}(n, q, \mathbb{H})$ & 4 & 1 & 1 & 1 & 3 & 16 & 2 \\
$\mathrm{SO}(2 n, \mathbb{R}) / \mathrm{U}(n, \mathbb{C})$ & 1 & 1 & 2 & 1 & 10 & 8 & 2 \\
$\mathrm{SU}(n, \mathbb{C}) / \operatorname{SO}(n, \mathbb{R})$ & 2 & 1 & 2 & 2 & 2 & 24 & 8 \\
$\mathrm{SU}(2 n, \mathbb{C}) / \mathrm{USp}(n, \mathbb{H})$ & 2 & 1 & 2 & 2 & 2 & 22 & 8 \\
$\mathrm{USp}(n, \mathbb{H}) / \mathrm{U}(n, \mathbb{C})$ & 4 & 1 & 2 & 1 & 3 & 17 & 2 \\
\hline
\end{tabular}

As the function $t \mapsto d_{\mathrm{TV}}\left(\mu_{t}\right.$, Haar) is non-increasing in $t$, the aspect of this function in the scale $t \propto \log n$ is then always as on Fig. 1. The constants $c$ and $C$ in Theorem 6 can be slightly improved by raising the integer $n_{0}$; and the restriction $n \geq n_{0}$ will only be used to ease certain computations and to get reasonable constants $c$ and $C$. On

Fig. 1 Aspect of the function $t \mapsto d_{\mathrm{TV}}\left(\mu_{t}\right.$, Haar $)$ for the Brownian motion on a classical simple compact Lie group or on a classical simple compact symmetric space

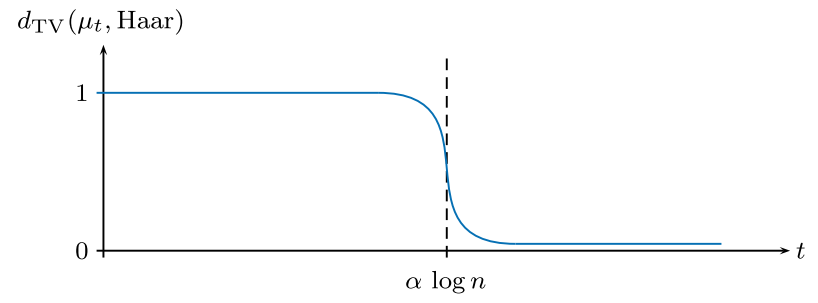


the other hand, notice that the constant $\alpha$ is equal to 2 for Lie groups, and to 1 for symmetric spaces.

Remark A proof of this result for compact symmetric spaces of rank 1 (the spheres and projective spaces) is given in [25], where the case of tori is also studied (as far as we know these were the only known cases of Theorem 6). As the case of tori is quite instructive, let us detail it a bit before giving the scheme of proof of Theorem 6 (again, see also [28]). The Fourier expansion of the heat kernel of the circle $\mathbb{T}=\mathbb{R} / 2 \pi \mathbb{Z}$ is

$$
p_{t}(x)=\frac{1}{\sqrt{2 \pi t}} \sum_{k \in \mathbb{Z}} \mathrm{e}^{-\frac{(x-k)^{2}}{2 t}}=\sum_{v \in \mathbb{Z}} \mathrm{e}^{-\frac{v^{2} t}{2}} \mathrm{e}^{\mathrm{i} v x}
$$

(see the examples after Theorem 11 ), so on the $n$-dimensional torus $\mathbb{T}^{(n)}$,

$p_{t}\left(x_{1}, \ldots, x_{n}\right)=\sum_{v \in \mathbb{Z}^{n}} \mathrm{e}^{-\frac{\|v\|^{2} t}{2}} \mathrm{e}^{\mathrm{i}\langle v \mid x\rangle} ;\left\|p_{t}-1\right\|_{\mathscr{L}^{2}}^{2}=\sum_{m=1}^{\infty}\left(\operatorname{card}\left\{v \in \mathbb{Z}^{n} \mid\|v\|^{2}=m\right\}\right) \mathrm{e}^{-m t}$.

For $m$ fixed, the number of integer-valued $n$-dimensional vectors with euclidian norm equal to $\sqrt{m}$ can clearly be bounded by $2^{m}$ times the number of ways of splitting $m$ into $n$ non-negative integers, that is to say $2^{m}\left(\begin{array}{c}n+m-1 \\ n-1\end{array}\right) \simeq(2 n)^{m}$. As a consequence, the $m$-th term of the series is of order $\frac{1}{n^{\varepsilon m}}$ if $t=(1+\varepsilon) \log n$, and $\left\|p_{t}-1\right\|_{\mathscr{L}^{2}}^{2} \rightarrow 0$ when $t=(1+\varepsilon) \log n$ and $n$ goes to infinity, at speed roughly of order $n^{-\varepsilon}$. We shall explain later how to deduce from this the cut-off in $\mathscr{L}^{p}$ for any $p \in[1,+\infty$ ) (see the proof of Theorem 7), so basically this is the proof of the cut-off phenomenon for Brownian motions on tori. Theorem 6 says that exactly the same happens for simple compact Lie groups; and the spirit of the proof is the same, but the quantities card $\{v \in$ $\mathbb{Z}^{n} \mid\|v\|^{2}=m$ \} get replaced by far more complex expressions, that are related to Weyl's formula for dimensions of irreducible representations of compact Lie groups.

A result similar to Theorem 6 has been proved by Rosenthal and Porod in [22-24] for random products of (real, or complex, or quaternionic) reflections. Our proofs are really inspired by their proofs, though quite different in the details of the computations. For the upper bound (Eq. 1.7), it has long been known that if $\lambda\left(X_{n}\right)$ denotes the spectral gap of the heat semigroup associated to the infinitesimal generator $L=\frac{1}{2} \Delta$, then for $n$ fixed, the total variation distance $d_{\mathrm{TV}}\left(\mu_{t}, \eta_{X_{n}}\right)$ decreases exponentially fast (see e.g. [19]):

$$
d_{\mathrm{TV}}\left(\mu_{t}, \eta_{X_{n}}\right) \leq C\left(X_{n}\right) \mathrm{e}^{-\lambda\left(X_{n}\right) t} .
$$

Consider now the family of spaces $\left(X_{n}\right)_{n \in \mathbb{N}}$, and assume that $C\left(X_{n}\right)=C n^{\delta}$, and that $\lambda\left(X_{n}\right)$ stays almost constant to $\lambda$-this last condition is ensured by the normalization (Eq. 1.3). Then, one obtains for $t=(1+\varepsilon) \frac{\delta}{\lambda} \log n$ the bound

$$
d_{\mathrm{TV}}\left(\mu_{t}, \eta_{X_{n}}\right) \leq \frac{C}{n^{\delta \varepsilon}} .
$$

Thus in theory, the upper bound (Eq. 1.7) follows from the calculations of the constants $C\left(X_{n}\right)$ and $\lambda\left(X_{n}\right)$ in each classical family. It is very hard to find directly a constant $C\left(X_{n}\right)$ that works for every time $t$. But on the other side, by using the representation theory of the classical simple compact Lie groups (cf. Section 2), one can determine series of negative exponentials that dominates the total variation 
distance; see Proposition 12. In these series, the "least negative" exponentials give the correct order of decay $\lambda\left(X_{n}\right)$. It remains then to prove that the other terms can be uniformly bounded. This is tedious, but doable, and these precise estimates are shown in Section 3: we shall adapt and improve the arguments of [6, 22-24].

A combination of the upper bound (Eq. 1.7) (which improves by a factor 2 the previously known results) and of the lower bound of Corollary 5 suffices to prove Theorem 6. Nonetheless, we give in Section 4 a new proof of the lower bound (Eq. 1.6), that do not require arguments of analysis on Riemannian manifolds (logSobolev estimates, etc.), and that is more closely related to the proof of the upper bound. This new proof relies on discriminating events, that have a probability close to 1 with respect to a marginal law $\mu_{t}$ with $t<t_{\text {cut-off }}$, and close to 0 with respect to the Haar measure. For instance, in the case of riffle shuffles, the sizes of the rising sequences of a permutation enable one to discriminate a random shuffle of order $k<k_{\text {cut-off }}$ from a uniform permutation; see [4, Section 2]. In the case of a Brownian motion on a classical compact Lie group, this is the trace of the matrices that allows to discriminate Haar distributed elements and random Brownian elements before cutoff time. Indeed, consider for instance a random unitary matrix $U_{n}$ of size $n$, taken under the Haar measure or under the marginal law $\mu_{t}$ of the Brownian motion at a given time $t$. Then, $\operatorname{tr} U_{n}$ is a complex valued random variable, and we shall see that

$$
\mathbb{E}\left[\left|\operatorname{tr} U_{n}-m\right|^{2}\right] \leq 1,
$$

where $m$ is the mean of $\operatorname{tr} U_{n}$; and this, for any $n \geq 1$ and any time $t \geq 0$ if $U_{n} \sim \mu_{t}$. However, $m=0$ under the Haar measure, whereas $|m| \gg 1$ for $t<t_{\text {cut-off. So, the }}$ trace of a Brownian unitary matrix before cut-off time will never "look the same" as the trace of an Haar distributed unitary matrix, see Fig. 2 hereafter.

Up to a minor modification, the same argument will work for special orthogonal groups and compact special orthogonal groups-in this later case, the trace of a quaternionic matrix of size $n$ is defined as the trace of the corresponding complex matrix of size $2 n$, cf. the remark at the end of Section 1.2. Over the classical simple compact symmetric spaces, the trace of matrices will be replaced by a zonal spherical function "of minimal non-zero weight"; these minimal zonal spherical functions are also those that give the order of decay of the series of negative exponentials that dominate $d_{\mathrm{TV}}\left(\mu_{t}\right.$, Haar) after the cut-off time. This argument for the lower bound

Fig. 2 Aspect of the density of the trace $\operatorname{tr} U_{n}$ of a random unitary matrix, with $U_{n} \sim$ Haar for the left peak, and $U_{n} \sim \mu_{t<t_{\text {cut-off }}}$ for the right peak (using Mathematica)

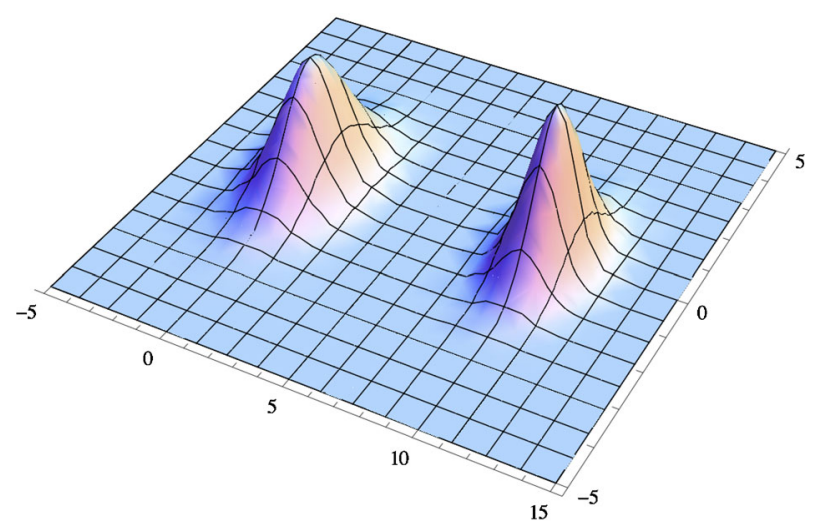


was already known, and it has been used successfully in [25] to prove the cut-off phenomenon over spheres, and the lower bound for compact Lie groups; we have extended it to the case of general compact symmetric spaces.

An important consequence of Theorem 6 and of its proof is that one also has a $\max -\mathscr{L}^{p}$-cut-off for every $p \in[1, \infty]$, and now with a known value of the mixing time.

Theorem 7 For every $p \in[1,+\infty]$, the family of Brownian motions $\left(\mathfrak{X}_{n}\right)_{n \in \mathbb{N}}$ traced on simple compact Lie groups $\left(K_{n}\right)_{n \in \mathbb{N}}$ in one of the three classical families (respectively, on simple compact symmetric spaces of type non-group $\left(X_{n}\right)_{n \in \mathbb{N}}$ in one of the seven classical families) has a max- $\mathscr{L}^{p}$-cut-off. If $p \in[1,+\infty)$, it is with respect to the sequence $t^{(n)}=2 \log n$ (respectively, $t^{(n)}=\log n$ ). If $p=+\infty$, it is with respect to the sequence $t^{(n)}=4 \log n$ (respectively, $t^{(n)}=2 \log n$ ).

Proof Since the Brownian motions considered are invariant by action of the isometry group, the max- $\mathscr{L}^{p}$-cut-offs are equivalent to simple $\mathscr{L}^{p}$-cut-offs for Brownian motions started from a fixed point. Then, the proof follows quite directly from Theorem 6 and from the previous works of Chen and Saloff-Coste. Thus,

- $\quad$ Theorem 6 clearly treats the case $p=1$.

- The upper bound of Theorem 6 will be shown by using Cauchy-Schwarz inequality and by estimating the $\mathscr{L}^{2}$-norm of $\left|p_{t}-1\right|$. Thus, from the very proof of Eq. 1.7, we shall get the same upper bounds for the $\mathscr{L}^{2}$-mixing times, and since they correspond to the lower bounds proven in [27, Theorem 1.1 and $1.2]$, the case $p=2$ will also be treated. Actually, it will be easy to get back these $\mathscr{L}^{2}$-lower bounds (for Lie groups and for symmetric spaces) directly from Proposition 12 , by looking at the term of the series expansion of $\left\|p_{t}-1\right\|_{\mathscr{L}^{2}}^{2}$ that corresponds to the discriminating weight in the sense of Lemma 13 and of Section 4.

- From [5, Theorem 5.3], we then get the two following facts: there is a $\mathscr{L}^{p}$-cut-off for every $p \in(1,+\infty]$ (because there is one for $p=2$, and the heat kernels are obviously self-adjoint), and the value of the mixing time in $\mathscr{L}^{\infty}$ is twice the value of the mixing time in $\mathscr{L}^{2}$. In particular, the case $p=+\infty$ is treated. Again, the computations of Section 3 allows one to recover this case, since the dominating series that will appear there have expansions almost identical to the expansions of $\left|p_{t}(e)-1\right|=d_{t}^{\infty}$.

- By Jensen's inequality, the cut-off times are increasing in $p$ (cf. the comparison theorem of mixing times [5, Proposition 5.1]), and they are here equal for $p=$ 1 and $p=2$, so they stay the same in between, and the case $p \in(1,2)$ is also treated.

- Finally, the only difficult case is when $p \in(2,+\infty)$. By using the fact that the logarithmic Sobolev constant is uniformly bounded from below for simple compact Lie groups and symmetric spaces, it follows again from the case $p=2$, see the arguments of $[25$, Sections 5 and 6 , in particular the third statement in Theorem 6.1] and of [26, Theorem 2.4.10].

The last case $p \in(2,+\infty)$ is the only place in this paper where advanced arguments of analysis of heat kernels on Riemannian manifolds are used and required. 


\subsection{Organization of the Paper}

In Section 2, we recall the basics of representation theory and harmonic analysis on compact symmetric spaces, with a particular emphasis on explicit formulas since we will need them in each case. All of it is really classical and of course wellknown by the experts, but it is necessary in order to fix the notations related to the harmonic analysis of the classical compact Lie groups and compact symmetric spaces. In Section 3, we use the explicit expansion of the densities to establish precise upper bounds on $\left\|p_{t}-1\right\|_{\mathscr{L}^{2}(X, \eta)}$; by Cauchy-Schwarz we obtain similar upper bounds on $d_{\mathrm{TV}}\left(\mu_{t}, \eta\right)$. The main idea is to control the growth of the dimension of an irreducible spherical representation involved in the expansion of $p_{t}$ when the corresponding highest weight grows in the lattice of weights (Section 3.2). The crucial fact, which was apparently unknown, is that precisely at cut-off time, the quantity

$$
\begin{cases}\left(D^{\lambda}\right)^{2} \mathrm{e}^{-t_{\text {cut-off }} B_{n}(\lambda)} & \text { in the group case, } \\ D^{\lambda} \mathrm{e}^{-t_{\text {cut-off }} B_{n}(\lambda)} & \text { in the non-group case }\end{cases}
$$

stays bounded for every $n$ and every $\lambda ; D^{\lambda}$ being the dimension of the irreducible or spherical irreducible representation of label $\lambda$, and $-B_{n}(\lambda)$ the associated eigenvalue of the Laplace-Beltrami operator. Combining this argument with a simple analysis of the generating series

$$
\sum_{\lambda \text { partition }} x^{|\lambda|}=\prod_{i \geq 1} \frac{1}{1-x^{i}}
$$

this is sufficient to get a correct upper bound after cut-off time.

Section 4 is then devoted to the (new) proof of the lower bounds. We use in each case a "minimal" zonal spherical function (the trace of matrices in the case of groups; see Section 4.1), and we compute its expectation and variance under Haar measure and Brownian measures (Section 4.2). A simple application of BienayméChebyshev's inequality will then show that the chosen zonal spherical function is indeed discriminating. An algebraic difficulty occurs in the case of symmetric spaces $G / K$ of type non-group, as one has to compute the expansion in zonal functions of the square of the discriminating zonal function, and this is far less obvious than in the case of irreducible characters. The problem is solved by writing the discriminating zonal function in terms of the coefficients of the matrices in the isometry group $G$, and by computing the joint moments of these coefficients under a Brownian measure. The combinations of negative exponentials appearing in these formulas are then in correspondence with the expansions of the squares of the discriminating zonal spherical functions.

\section{Fourier Expansion of the Densities}

In this section, we explain how to compute the density $p_{t}^{K}(k)$ or $p_{t}^{X}(x)$ of the marginal law $\mu_{t}$ of the Brownian motion traced on a classical compact symmetric space. This computation is done in an abstract setting for instance in [19] or [2], and we shall give 
at the end of this section its concrete counterpart in each classical case, see Theorem

11. The main ingredients of the computation are:

1. Peter-Weyl's theorem and its refinement due to Cartan, that ensures that the matrix coefficients of the irreducible representations of $K$ (respectively, of the irreducible spherical representations of $G$ ) form an orthogonal basis of $\mathscr{L}^{2}(K, \eta)$ (respectively, of $\mathscr{L}^{2}(G / K, \eta)$ ); see Section 2.1.

2. the classical highest weight theory, that describes the irreducible representations of a compact simple Lie group and give formulas for their dimensions and characters; see Section 2.2.

On these subjects, we refer to the two books by Helgason [14, 15], and also to $[3,10,11,13,29]$ for the representation theory of compact Lie groups. We shall only recall what is needed in order to have a good understanding of the formulas of Theorem 11. We shall also fix all the notations related to the harmonic analysis on (classical) compact symmetric spaces.

\subsection{Peter-Weyl's Theorem and Cartan's Refinement}

Let $K$ be a compact (Lie) group, and $\widehat{K}$ be the set of isomorphism classes of irreducible complex linear representations of $K$. Each class $\lambda \in \widehat{K}$ is finite-dimensional, and we shall denote $V^{\lambda}$ the corresponding complex vector space; $\rho^{\lambda}: K \rightarrow \mathrm{U}\left(V^{\lambda}\right)$ the representation morphism; $D^{\lambda}=\operatorname{dim}_{\mathbb{C}} V^{\lambda}$ the dimension of the representation; $\chi^{\lambda}(\cdot)=\operatorname{tr} \rho^{\lambda}(\cdot)$ the character; and $\hat{\chi}^{\lambda}(\cdot)=\chi^{\lambda}(\cdot) / D^{\lambda}$ the normalized character. An Hermitian scalar product on $\operatorname{End}\left(V^{\lambda}\right)$ is $\langle M \mid N\rangle_{\operatorname{End}\left(V^{\lambda}\right)}=D^{\lambda} \operatorname{tr}\left(M^{\dagger} N\right)$. For every class $\lambda$ and every function $f \in \mathscr{L}^{2}(K)$, we set

$$
\widehat{f}(\lambda)=\int_{K} f(k) \rho^{\lambda}(k) d k
$$

this is an element of $\operatorname{End}\left(V^{\lambda}\right)$. We refer to $[3,10]$ for a proof of the following results.

Theorem 8 (Peter-Weyl) The (non-commutative) Fourier transform $\mathcal{F}: f \mapsto$ $\sum_{\lambda \in \widehat{K}} \widehat{f}(\lambda)$ realizes an isometry and an isomorphism of (non-unital) algebras between $\mathscr{L}^{2}(K, \eta)$ and $\bigoplus_{\lambda \in \widehat{K}} \operatorname{End}\left(V^{\lambda}\right)$. So, if $f \in \mathscr{L}^{2}(K)$, then

$$
\begin{aligned}
f(k) & =\sum_{\lambda \in \widehat{K}} D^{\lambda} \operatorname{tr}\left(\tilde{f}(\lambda) \rho^{\lambda}(k)\right)=\sum_{\lambda \in \widehat{K}} D^{\lambda} \operatorname{tr}\left(\int_{K} f\left(h^{-1} k\right) \rho^{\lambda}(h) d h\right) \\
\|f\|_{\mathscr{L}^{2}(K)}^{2} & =\sum_{\lambda \in \widehat{K}}\|\widehat{f}(\lambda)\|_{\operatorname{End}\left(V^{\lambda}\right)}^{2}=\sum_{\lambda \in \widehat{K}} D^{\lambda} \operatorname{tr}\left(\widehat{f}(\lambda)^{\dagger} \widehat{f}(\lambda)\right)
\end{aligned}
$$

where $\tilde{f}(\lambda)=\widehat{f^{-}}(\lambda)=\int_{K} f\left(k^{-1}\right) \rho^{\lambda}(k) d k$.

Assume now that $f$ is in $\mathscr{L}^{2}(K, \eta)^{K}$, the subalgebra of conjugacy-invariant functions. The Fourier expansion (Eq. 2.1) and the Parseval identity (Eq. 2.2) become then

$$
f(k)=\sum_{\lambda \in \widehat{K}}\left(D^{\lambda}\right)^{2} \widehat{\chi}^{\lambda}\left(f^{-}\right) \widehat{\chi}^{\lambda}(k) \quad ; \quad\|f\|_{\mathscr{L}^{2}(K)}^{2}=\sum_{\lambda \in \widehat{K}}\left|\chi^{\lambda}(f)\right|^{2},
$$


and in particular, the irreducible characters $\chi^{\lambda}$ form an orthonormal basis of $\mathscr{L}^{2}(K)^{K}$. Cartan gave a statement generalizing Theorem 8 for $\mathscr{L}^{2}(G / K, \eta)$, where $X=G / K$ is a simply connected irreducible compact symmetric space. Call spherical an irreducible representation $\left(V^{\lambda}, \rho^{\lambda}\right)$ of $G$ such that $\left(V^{\lambda}\right)^{K}$, the space of vectors invariant by $\rho^{\lambda}(K)$, is non-zero. Then, it is in fact one-dimensional, so one can find a vector $e^{\lambda}$ of norm $\left\|e^{\lambda}\right\|^{2}=1$, unique up to multiplication by $z \in \mathbb{T}$, such that $\left(V^{\lambda}\right)^{K}=\mathbb{C} e^{\lambda}$. Denote then $\mathscr{C}^{\lambda}(G / K)$ the set of functions from $G$ to $\mathbb{C}$ that can be written as

$$
f(g)=f_{v}(g)=\left\langle v \mid \rho^{\lambda}(g)\left(e^{\lambda}\right)\right\rangle \quad \text { with } v \in V^{\lambda} .
$$

Such a function is right- $K$-invariant, so it can be considered as a function from $G / K$ to $\mathbb{C}$.

Theorem 9 (Cartan) Let $\widehat{G}^{K}$ be the set of spherical irreducible representations of $G$. The space $\mathscr{L}^{2}(G / K, \eta)$ is isometric to the orthogonal sum $\bigoplus_{\lambda \in \widehat{G}^{K}} \mathscr{C}^{\lambda}(G / K)$. This decomposition corresponds to the Fourier expansion

$$
f(g K)=\sum_{\lambda \in \widehat{G}^{K}} D^{\lambda} \operatorname{tr}\left(\int_{G} f\left(h^{-1} g K\right) \rho^{\lambda}(h) d h\right)
$$

for $f \in \mathscr{L}^{2}(G / K)$.

In each space $\mathscr{C}^{\lambda}(G / K)$, the space of left $K$-invariant functions is onedimensional, and it is generated by the zonal spherical function $\phi^{\lambda}(g K)=$ $\left\langle e^{\lambda}\left|\rho^{\lambda}(g)\left(e^{\lambda}\right)\right|\right.$. These spherical functions form an orthogonal basis of $\mathscr{L}^{2}(X)^{K}$ when $\lambda$ runs over spherical representations. So, a $K$-invariant function writes as

$$
f(g K)=\sum_{\lambda \in \widehat{G}^{K}} D^{\lambda} \phi^{\lambda}\left(f^{-}\right) \phi^{\lambda}(g K),
$$

where $\phi^{\lambda}(f)=\int_{G / K} f(x) \phi^{\lambda}(x) d x=\left\langle e^{\lambda} \mid \int_{G} f(g K) \rho^{\lambda}(g)\left(e^{\lambda}\right) d g\right\rangle$.

To conclude with, notice that the decomposition of Theorem 9 is the decomposition of $\mathscr{L}^{2}(G / K, \eta)$ in common eigenspaces of the elements of $\mathscr{D}(G / K)$, the commutative algebra of $G$-invariant differential operators on $X$. Thus, there are morphisms of algebras $c^{\lambda}: \mathscr{D}(G / K) \rightarrow \mathbb{C}$ such that

$$
L\left(f^{\lambda}\right)=c^{\lambda}(L) f^{\lambda}
$$

for every $\lambda \in \widehat{G}^{K}$, every $L \in \mathscr{D}(G / K)$ and every $f^{\lambda} \in \mathscr{C}^{\lambda}(G / K)$.

\subsection{Highest Weight Theorem and Weyl's Character Formula}

The theory of highest weights of representations enables us to identify $\widehat{K}$ or $\widehat{G}^{K}$, and to compute the coefficients $c^{\lambda}(\Delta)$ associated to the Laplace-Beltrami operator. If $G$ is a connected compact Lie group, its maximal tori are all conjugated, and every element of $K$ is contained in a maximal torus $T$. Denote $W=\operatorname{Norm}(T) / T$ the Weyl group of $G$, and call weight of a representation $V$ of $G$ an element of $t^{*}$, or equivalently a group morphism $\omega: T \rightarrow \mathbb{T}$ such that $V^{\omega}=\{v \in V \mid \forall t \in T, t \cdot v=$ $\omega(t) \cdot v\} \neq 0$. Every representation $V$ of $G$ is the direct sum of its weight subspaces $V^{\omega}$, and this decomposition is always $W$-invariant. Besides, the set of all weights of 
all representations of $G$ is a lattice $\mathbb{Z} \Omega$ whose rank is also the dimension of $T$. We take a $W$-invariant scalar product on the real vector space $\mathbb{R} \Omega=\mathbb{Z} \Omega \otimes_{\mathbb{Z}} \mathbb{R}$, e.g., the dual of the scalar product given by Eq. 1.3, where $\mathbb{R} \Omega$ is identified with $t^{*}$ by mean of $\omega \mapsto d_{e} \omega$ for $\omega \in \mathbb{Z} \Omega$. We also fix a closed fundamental set $C$ for the action of the Weyl group on $\mathbb{R} \Omega$. We call dominant a weight $\omega$ that falls in the Weyl chamber $C$. A root of $G$ is a non-zero weight of the adjoint representation. The set of roots $\Phi$ is a root system, which means that certain combinatorial relations are satisfied between its elements. There is a unique way to split $\Phi$ in a set $\Phi_{+}$of positive roots and a set $\Phi_{-}=-\Phi_{+}$such that

$$
C=\left\{x \in \mathbb{R} \Omega \mid \forall \alpha \in \Phi_{+},\langle x \mid \alpha\rangle \geq 0\right\} .
$$

Call simple a positive root $\alpha$ that cannot be written as the sum of two positive roots; and simple coroot an element $\check{\alpha}=\frac{2 \alpha}{\langle\alpha \mid \alpha\rangle}$ with $\alpha$ simple root. Then, a distinguished basis of the lattice $\mathbb{Z} \Omega$ is given by the fundamental weights $\varpi_{1}, \varpi_{2}, \ldots, \varpi_{r}$, the dual basis of the basis of coroots. Hence, the sets of weights and of dominant weights have the following equivalent descriptions:

$$
\begin{array}{r}
\mathbb{Z} \Omega=\bigoplus_{i=1}^{r} \mathbb{Z} \varpi_{i}=\left\{x \in \mathbb{R} \Omega \mid \forall \alpha \in \Phi, \frac{\langle x \mid \alpha\rangle}{\langle\alpha \mid \alpha\rangle} \in \mathbb{Z}\right\} ; \\
\operatorname{Dom}(\mathbb{Z} \Omega)=\bigoplus_{i=1}^{r} \mathbb{N} \varpi_{i}=\left\{x \in \mathbb{R} \Omega \mid \forall \alpha \in \Phi, \frac{\langle x \mid \alpha\rangle}{\langle\alpha \mid \alpha\rangle} \in \mathbb{N}\right\} .
\end{array}
$$

Suppose now that $G$ is a semi-simple simply connected compact Lie group, and consider the partial order induced by the convex set $C$ on $\mathbb{R} \Omega$. Recall that the Weyl group $W$ is a Coxeter group generated by the symmetries along the simple roots $\alpha_{1}, \alpha_{2}, \ldots, \alpha_{r}$; so in particular, it admits a signature morphism $\varepsilon: W \rightarrow\{ \pm 1\}$. Weyl's theorem ensures that every irreducible representation $V$ of $G$ has a unique highest weight $\omega_{0}$ for this order, which is then of multiplicity one and determines the isomorphism class of $V$. Moreover, the restriction to $T$ of the irreducible character associated to a dominant weight $\lambda$ is given by

$$
\chi^{\lambda}(t)=\frac{\sum_{\sigma \in W} \varepsilon(\sigma) \sigma(\lambda+\rho)(t)}{\sum_{\sigma \in W} \varepsilon(\sigma) \sigma(\rho)(t)},
$$

where $\rho$ is the half-sum of all positive roots, or equivalently the sum of the fundamental weights. This formula degenerates into the dimension formula

$$
D^{\lambda}=\operatorname{dim} V^{\lambda}=\frac{\prod_{\alpha \in \Phi_{+}}\langle\lambda+\rho \mid \alpha\rangle}{\prod_{\alpha \in \Phi_{+}}\langle\rho \mid \alpha\rangle} .
$$

These results make Eq. 2.1 essentially explicit in the case of a conjugacy invariant function on a semi-simple compact Lie group $K$; in particular, we shall see in a moment that the highest weights are labelled by partitions or similar combinatorial objects in all the classical cases.

The case of a compact symmetric space $X=G / K$ of type non-group is a little more involved. Denote $\theta$ an involutive automorphism of a semi-simple simply connected compact Lie group $G$, with $K=G^{\theta}$. Set $P=\left\{g \in G \mid \theta(g)=g^{-1}\right\}$; one has then the Cartan decomposition $G=K P$. In addition to the previous assumptions, 
one assumes that the maximal torus $T \subset G$ is chosen so that $\theta(T)=T$ and $P \cap T$ is a maximal torus in $P$ (one can always do so up to conjugation of the torus). Then, Cartan-Helgason theorem ([15, Theorem 4.1]) says that the spherical representations in $\widehat{G}^{K}$ are precisely the irreducible representations in $\widehat{G}$ that are trivial on $K \cap T=$ $T^{\theta}$. This subgroup $T^{\theta}$ of $T \simeq \mathbb{T}^{r}$ is always the product of a subtorus $\mathbb{T}^{s \leq r}$ with an elementary abelian 2 -group $(\mathbb{Z} / 2 \mathbb{Z})^{t}$; this will correspond to additional conditions on the size and the parity of the parts of the partitions labeling the highest weights in $\widehat{G}^{K}$ (in comparison to $\widehat{G}$ ), cf. Section 2.3. The corresponding zonal spherical functions $\phi^{\lambda}$ do not have in general an expression as simple as Eq. 2.5; see however [16, Part 1]. For most of our computations, this will not be a problem, since we shall only use certain properties of the spherical functions-e.g., their orthogonality and the formula for the dimension $D^{\lambda}$ —and not their explicit form; see however Section 4.1.

The last ingredient in the computation of the densities is the value of the coefficient $c^{\lambda}(\Delta)$ such that

$$
\frac{\Delta\left(f^{\lambda}\right)}{2}=c^{\lambda}(\Delta) f^{\lambda}
$$

for every function $f^{\lambda}$ either in $\mathscr{R}^{\lambda}(K)=\operatorname{Vect}\left(\left\{k \mapsto\left(\rho^{\lambda}(k)\right)_{i j}, \quad 1 \leq i, j \leq D^{\lambda}\right\}\right)$ in the group case, or in $\mathscr{C}^{\lambda}(G / K)$ in the case of a symmetric space. In the group case, by comparing the definition of the Casimir operator 1.5 with the definition of the Laplace-Beltrami operator 1.4 , one sees that $2 c^{\lambda}(\Delta)$ is also $\kappa_{\lambda}$, the constant by which the Casimir operator $C$ acts via the infinitesimal representation $d \rho^{\lambda}: U(\mathfrak{k}) \rightarrow$ $\operatorname{End}\left(V^{\lambda}\right) — \mathrm{cf}$. the remark at the end of Section 1.3. This constant is equal to

$$
\kappa_{\lambda}=-\langle\lambda+2 \rho \mid \lambda\rangle,
$$

see [2, Eq. 3.4] and the references therein, or [18] and [10, Chapter12] for a caseby-case computation. These later explicit computations follow from the following expressions of the Casimir operators (see [18, Lemma 1.2]):

$$
\begin{aligned}
C_{\mathfrak{s o}(n)}= & \sum_{1 \leq i<j \leq n}\left(\frac{E_{i j}-E_{j i}}{\sqrt{n}}\right)^{\otimes 2} \\
C_{\mathfrak{s u}(n)=} & \frac{1}{n} \sum_{i=1}^{n} \mathrm{i} E_{i i} \otimes \mathrm{i} E_{i i}-\frac{1}{n^{2}} \sum_{i, j=1}^{n} \mathrm{i} E_{i i} \otimes \mathrm{i} E_{j j}+\sum_{1 \leq i<j \leq n}\left(\frac{E_{i j}-E_{j i}}{\sqrt{2 n}}\right)^{\otimes 2}+\left(\frac{\mathrm{i} E_{i j}+\mathrm{i} E_{j i}}{\sqrt{2 n}}\right)^{\otimes 2} \\
C_{\mathfrak{u} \mathfrak{s p}(n)=} & \frac{1}{2 n} \sum_{i=1}^{n} \mathrm{i} E_{i i} \otimes \mathrm{i} E_{i i}+\mathrm{j} E_{i i} \otimes \mathrm{j} E_{i i}+\mathrm{k} E_{i i} \otimes \mathrm{k} E_{i i} \\
& +\sum_{1 \leq i<j \leq n}\left(\frac{E_{i j}-E_{j i}}{\sqrt{4 n}}\right)^{\otimes 2}+\left(\frac{\mathrm{i} E_{i j}+\mathrm{i} E_{j i}}{\sqrt{4 n}}\right)^{\otimes 2}+\left(\frac{\mathrm{j} E_{i j}+\mathrm{j} E_{j i}}{\sqrt{4 n}}\right)^{\otimes 2}+\left(\frac{\mathrm{k} E_{i j}+\mathrm{k} E_{j i}}{\sqrt{4 n}}\right)^{\otimes 2}
\end{aligned}
$$

where $E_{i j}$ are the elementary matrices in $\mathrm{M}(n, k)$ with $k=\mathbb{R}, \mathbb{C}$ or $\mathbb{H}$-beware that the tensor product are over $\mathbb{R}$, since we deal with real Lie algebras.

In the case of a compact symmetric space, the same formula 2.7 gives the action of $\Delta^{G / K}$ on $\mathscr{C}^{\lambda}(G / K)$. Indeed, remember that the Riemannian structures on $G$ and $G / K$ are chosen in such a way that for any $f \in \mathscr{C}^{\infty}(G)$ that is right $K$-invariant, $\Delta^{G / K}(f)(g K)=\Delta^{G}(f)(g)$. Consider then a function in $\mathscr{C}^{\lambda}(G / K)$, viewed as a 
function on $G$. In definition 2.3, $f$ appears clearly as a linear combination of matrix coefficients of the spherical representation $\lambda$, so the previous discussion holds.

\subsection{Densities of a Brownian Motion with Values in a Compact Symmetric Space}

Let us now see how the previous results can be used to compute the density $p_{t}^{K}(k)$ or $p_{t}^{X}(x)$ of a Brownian motion on a compact Lie group or symmetric space. These densities are in both cases $K$-invariant, so they can be written as

$$
p_{t}^{K}(k)=\sum_{\lambda \in \widehat{K}} a_{\lambda}(t) \widehat{\chi}^{\lambda}(k) \quad \text { or } \quad p_{t}^{X}(x)=\sum_{\lambda \in \widehat{G}^{K}} a_{\lambda}(t) \phi^{\lambda}(x)
$$

by using either Peter-Weyl's theorem in the case of conjugacy-invariant functions on $K$, or Cartan's theorem in the case of left $K$-invariant functions on $G / K$. We then apply $\frac{\Delta}{2}=\left.\frac{d P_{t}}{d t}\right|_{t=0}$ to these formulas:

$$
\frac{\Delta p_{t}^{K}(k)}{2}=\sum_{\lambda \in \widehat{K}} \frac{\kappa_{\lambda}}{2} a_{\lambda}(t) \widehat{\chi}^{\lambda}(k)=\frac{d p_{t}^{K}(t)}{d t}=\sum_{\lambda \in \widehat{K}} \frac{d a_{\lambda}(t)}{d t} \widehat{\chi}^{\lambda}(k),
$$

and similarly in the case of a compact symmetric space. Thus, $\frac{d a_{\lambda}(t)}{d t}=\frac{\kappa_{\lambda}}{2} a_{\lambda}(t)$ and $a_{\lambda}(t)=a_{\lambda}(0) \mathrm{e}^{\frac{\kappa_{\lambda}}{2} t}$ for every class $\lambda$. The coefficient $a_{\lambda}(0)$ is given in the group case by

$$
a_{\lambda}(0)=\left(D^{\lambda}\right)^{2} \int_{K} \widehat{\chi}^{\lambda}(k) \delta_{e_{K}}(d k)=\left(D^{\lambda}\right)^{2} \widehat{\chi}^{\lambda}\left(e_{K}\right)=\left(D^{\lambda}\right)^{2}
$$

and in the case of a compact symmetric space of type non-group by

$$
a_{\lambda}(0)=D^{\lambda}\left\langle e^{\lambda} \mid \int_{G} \rho^{\lambda}(g)\left(e^{\lambda}\right) \delta_{e_{G}}(d g)\right\rangle=D^{\lambda} \phi^{\lambda}\left(e_{G}\right)=D^{\lambda} .
$$

Proposition 10 The density of the law $\mu_{t}$ of the Brownian motion traced on a classical simple compact Lie group $K$ is

$$
p_{t}^{K}(k)=\sum_{\lambda \in \widehat{K}} \mathrm{e}^{-\frac{t}{2}\langle\lambda+2 \rho \mid \lambda\rangle}\left(\frac{\prod_{\alpha \in \Phi_{+}}\langle\lambda+\rho \mid \alpha\rangle}{\prod_{\alpha \in \Phi_{+}}\langle\rho \mid \alpha\rangle}\right)^{2} \hat{\chi}^{\lambda}(k),
$$

and the density of the Brownian motion traced on a classical simple compact symmetric space $G / K$ is

$$
p_{t}^{X}(x)=\sum_{\lambda \in \widehat{G}^{K}} \mathrm{e}^{-\frac{t}{2}\langle\lambda+2 \rho \mid \lambda\rangle}\left(\frac{\prod_{\alpha \in \Phi_{+}}\langle\lambda+\rho \mid \alpha\rangle}{\prod_{\alpha \in \Phi_{+}}\langle\rho \mid \alpha\rangle}\right) \phi^{\lambda}(x) .
$$

Let us now apply this in each classical case. We refer to [3], [11, Chapter 24] and [14, Chapter 10] for most of the computations. Unfortunately, we have not found a reference which describes explicitly the spherical representations; this explains the following long discussion. For convenience, we shall assume:

- $n \geq 2$ when considering $\mathrm{SU}(n), \mathrm{SU}(n) / \mathrm{SO}(n), \mathrm{SU}(2 n) / \mathrm{USp}(n)$ or $\mathrm{SU}(n) / \mathrm{S}$ $(\mathrm{U}(n-q) \times \mathrm{U}(q))$; 
- $n \geq 3$ when considering $\operatorname{USp}(n), \operatorname{USp}(n) / \mathrm{U}(n)$ or $\operatorname{USp}(n) /(\operatorname{USp}(n-q) \times$ $\mathrm{USp}(q))$

- $\quad n \geq 10$ when considering $\mathrm{SO}(n), \mathrm{SO}(2 n) / \mathrm{U}(n)$ or $\mathrm{SO}(n) /(\mathrm{SO}(n-q) \times \mathrm{SO}(q))$.

For $\mathrm{SU}(2 n) / \mathrm{USp}(n)$ and $\mathrm{SO}(2 n) / \mathrm{U}(n)$, the restriction will hold on the " $2 n$ " parameter of the group of isometries. These assumptions shall ensure that the root systems and the Schur functions of type B, C and D are not degenerate, and later this will ease certain computations. For Grassmanian varieties, we shall also suppose by symmetry that $q \leq\left\lfloor\frac{n}{2}\right\rfloor$.

\subsubsection{Special Unitary Groups and their Quotients}

In $\operatorname{SU}(n, \mathbb{C})$, a maximal torus is

$$
T=\left\{\operatorname{diag}\left(z_{1}, z_{2}, \ldots, z_{n}\right) \mid \forall i, z_{i} \in \mathbb{T} \text { and } \prod_{i=1}^{n} z_{i}=1\right\}=\mathbb{T}^{n} / \mathbb{T},
$$

and the Weyl group is the symmetric group $\mathfrak{S}_{n}$. The simple roots and the fundamental weights, viewed as elements of $\mathfrak{t}^{*}$, are $\alpha_{i}=e^{i}-e^{i+1}$ and

$$
\varpi_{i}=\frac{n-i}{n}\left(e^{1}+\cdots+e^{i}\right)-\frac{i}{n}\left(e^{i+1}+\cdots+e^{n}\right)
$$

for $i \in \llbracket 1, n-1 \rrbracket$, where $e^{i}$ is the coordinate form on $t=i \mathbb{R}^{n}$ defined by $e^{i}\left(\operatorname{diag}\left(\mathrm{i} t_{1}, \mathrm{i} t_{2}, \ldots, \mathrm{i} t_{n}\right)\right)=t_{i}$. The dominant weights are then the

$$
\left(\lambda_{1}-\lambda_{2}\right) \varpi_{1}+\cdots+\lambda_{n-1} \varpi_{n-1}=\lambda_{1} e^{1}+\cdots+\lambda_{n-1} e^{n-1}-|\lambda| \frac{\varpi_{n}}{n},
$$

where $\lambda=\left(\lambda_{1} \geq \lambda_{2} \geq \cdots \geq \lambda_{n-1}\right)$ is any partition (non-increasing sequence of nonnegative integers) of length $(n-1)$; it is then convenient to set $\lambda_{n}=0$. The half-sum of positive roots is given by $2 \rho=2\left(\varpi_{1}+\cdots+\varpi_{n-1}\right)=\sum_{i=1}^{n}(n+1-2 i) e^{i}$, and the scalar product on $\mathfrak{t}^{*}$ is $\frac{1}{n}$ times the usual euclidian scalar product $\left\langle e^{i} \mid e^{j}\right\rangle=\delta_{i j}$. So,

$$
D^{\lambda}=\prod_{1 \leq i<j \leq n} \frac{\lambda_{i}-\lambda_{j}+j-i}{j-i} \quad ; \quad \chi^{\lambda}(k)=s_{\lambda}\left(z_{1}, \ldots, z_{n}\right)=\frac{\operatorname{det}\left(z_{i}^{\lambda_{j}+n-j}\right)_{1 \leq i, j \leq n}}{\operatorname{det}\left(z_{i}^{n-j}\right)_{1 \leq i, j \leq n}},
$$

where $z_{1}, \ldots, z_{n}$ are the eigenvalues of $k$; thus, characters are given by Schur functions. The Casimir coefficient is

$$
-\kappa_{\lambda}=-\frac{|\lambda|^{2}}{n^{2}}+\frac{1}{n} \sum_{i=1}^{n} \lambda_{i}^{2}+(n+1-2 i) \lambda_{i},
$$

where $|\lambda|=\sum_{i=1}^{n} \lambda_{i}$ denotes the size of the partition.

Though we have chosen to examine only the Brownian motions on simple Lie groups, the same work can be performed over the unitary groups $\mathrm{U}(n, \mathbb{C})$, which are reducible Lie groups. Irreducible representations of $\mathrm{U}(n, \mathbb{C})$ are labelled by sequences $\lambda=\left(\lambda_{1} \geq \cdots \geq \lambda_{n}\right)$ in $\mathbb{Z}^{n}$, the action of the torus $\mathbb{T}^{n}$ on a corresponding highest weight vector being given by the morphism $\lambda\left(z_{1}, \ldots, z_{n}\right)=z_{1}^{\lambda_{1}} \cdots z_{n}^{\lambda_{n}}$. The dimensions and characters are the same as before, and the Casimir coefficient is $\frac{1}{n} \sum_{i=1}^{n} \lambda_{i}^{2}+(n+1-2 i) \lambda_{i}$. 
For the spaces of quaternionic structures $\mathrm{SU}(2 n, \mathbb{C}) / \mathrm{USp}(n, \mathbb{H})$, the involutive automorphism defining the symmetric pair is $\theta(g)=J_{2 n} \bar{g} J_{2 n}^{-1}$, where $J_{2 n}$ is the skew symmetric matrix

$$
J_{2 n}=\left(\begin{array}{ccccc}
0 & 1 & & & \\
-1 & 0 & & & \\
& & \ddots & & \\
& & & 0 & 1 \\
& & & -1 & 0
\end{array}\right)
$$

of size $2 n$. The subgroup $T^{\theta}$ is the set of matrices $\operatorname{diag}\left(z_{1}, z_{1}^{-1}, \ldots, z_{n}, z_{n}^{-1}\right)$, with all the $z_{i}$ 's in $\mathbb{T}$. The dominant weights $\lambda$ trivial on $T^{\theta}$ correspond then to partitions will all parts doubled:

$$
\forall i \in \llbracket 1, n \rrbracket, \quad \lambda_{2 i-1}=\lambda_{2 i} .
$$

In the spaces of real structures $\mathrm{SU}(n, \mathbb{C}) / \mathrm{SO}(n, \mathbb{R}), \theta(g)=\bar{g}$. The intersection of the torus with $\mathrm{SO}(n, \mathbb{R})$ is isomorphic to $(\mathbb{Z} / 2 \mathbb{Z})^{n} /(\mathbb{Z} / 2 \mathbb{Z})$, and therefore, by Cartan-Helgason theorem, the spherical representations correspond to partitions with even parts:

$$
\forall i \in \llbracket 1, n \rrbracket, \quad \lambda_{i} \equiv 0 \bmod 2 .
$$

Finally, for the complex Grassmannian varieties $\mathrm{SU}(n, \mathbb{C}) / \mathrm{S}(\mathrm{U}(n-q, \mathbb{C}) \times$ $\mathrm{U}(q, \mathbb{C}))$, it is a little simpler to work with $\mathrm{U}(n, \mathbb{C}) /(\mathrm{U}(n-q, \mathbb{C}) \times \mathrm{U}(q, \mathbb{C}))$, which is the same space. An involutive automorphism defining the symmetric pair is then $\theta(g)=K_{n, q} g K_{n, q}$, where

$$
K_{n, q}=\left(\begin{array}{cc}
T_{q} \\
T_{q} & I_{n-2 q}
\end{array}\right)
$$

and $T_{q}$ is the $(q \times q)$-anti-diagonal matrix with entries 1 on the anti-diagonal. The subgroup $T^{\theta}$ is then the set of diagonal matrices $\operatorname{diag}\left(z_{1}, \ldots, z_{q}, z_{q+1}, \ldots, z_{n-q}\right.$, $\left.z_{q}, \ldots, z_{1}\right)$ with the $z_{i}$ 's in $\mathbb{T}$. The dominant weights $\lambda$ trivial on $T^{\theta}$ correspond then to partitions of length $q$, written as

$$
\lambda=\left(\lambda_{1}, \ldots, \lambda_{q}, 0, \ldots, 0,-\lambda_{q}, \ldots,-\lambda_{1}\right) .
$$

\subsubsection{Compact Symplectic Groups and their Quotients}

Considering $\operatorname{USp}(n, \mathbb{H})$ as a subgroup of $\operatorname{SU}(2 n, \mathbb{C})$, a maximal torus is

$$
T=\left\{\operatorname{diag}\left(z_{1}, z_{1}^{-1}, \ldots, z_{n}, z_{n}^{-1}\right) \mid \forall i, z_{i} \in \mathbb{T}\right\},
$$

and the Weyl group is the hyperoctahedral group $\mathfrak{H}_{n}=(\mathbb{Z} / 2 \mathbb{Z})_{2} \mathfrak{S}_{n}$. The simple roots, viewed as elements of $\mathfrak{t}^{*}$, are $\alpha_{i}=e^{i}-e^{i+1}$ for $i \in \llbracket 1, n-1 \rrbracket$ and $\alpha_{n}=$ $2 e^{n}$; and the fundamental weights are $\varpi_{i}=e^{1}+\cdots+e^{i}$ for $i \in \llbracket 1, n \rrbracket$. Here, $e^{i}\left(\operatorname{diag}\left(\mathrm{i} t_{1},-\mathrm{i} t_{1}, \ldots, \mathrm{i} t_{n},-\mathrm{i} t_{n}\right)\right)=t_{i}$. The dominant weights can therefore be written 
as $\lambda_{1} e^{1}+\cdots+\lambda_{n} e^{n}$, where $\lambda=\left(\lambda_{1} \geq \lambda_{2} \geq \cdots \geq \lambda_{n}\right)$ is any partition of length $n$. This leads to

$$
\begin{aligned}
D^{\lambda} & =\prod_{1 \leq i<j \leq n} \frac{\lambda_{i}-\lambda_{j}+j-i}{j-i} \prod_{1 \leq i \leq j \leq n} \frac{\lambda_{i}+\lambda_{j}+2 n+2-i-j}{2 n+2-i-j} ; \\
\chi^{\lambda}(k) & =s c_{\lambda}\left(z_{1}, z_{1}^{-1}, \ldots, z_{n}, z_{n}^{-1}\right)=\frac{\operatorname{det}\left(z_{i}^{\lambda_{j}+n-j+1}-z_{i}^{-\left(\lambda_{j}+n-j+1\right)}\right)_{1 \leq i, j \leq n}}{\operatorname{det}\left(z_{i}^{n-j+1}-z_{i}^{-(n-j+1)}\right)_{1 \leq i, j \leq n}},
\end{aligned}
$$

where $z_{1}^{ \pm 1}, \ldots, z_{n}^{ \pm 1}$ are the eigenvalues of $k$ viewed as a matrix in $\operatorname{SU}(2 n, \mathbb{C})$. The Casimir coefficient is $-\kappa_{\lambda}=\frac{1}{2 n} \sum_{i=1}^{n} \lambda_{i}^{2}+(2 n+2-2 i) \lambda_{i}$.

In the spaces of complex structures $\operatorname{USp}(n, \mathbb{H}) / \mathrm{U}(n, \mathbb{C}), \theta(g)=\bar{g}$ (inside $\mathrm{SU}(2 n, \mathbb{C}))$. The subgroup $T^{\theta}$ is isomorphic to $(\mathbb{Z} / 2 \mathbb{Z})^{n}$, so the spherical representations correspond here again to partitions with even parts. On the other hand, for quaternionic Grassmannian varieties $\operatorname{USp}(n, \mathbb{H}) /(\mathrm{USp}(n-q, \mathbb{H}) \times \operatorname{USp}(q, \mathbb{H}))$, a choice for the involutive automorphism is $\theta(g)=L_{2 n, q} g L_{2 n, q}$, where

$$
L_{2 n, q}=\left(\begin{array}{cccc}
T_{4} & & & \\
& \ddots & & \\
& & T_{4} & \\
& & & I_{2 n-4 q}
\end{array}\right) \text {, }
$$

$T_{4}$ appearing $q$ times (with all the computations made inside $\mathrm{SU}(2 n, \mathbb{C})$ ). Then, $T^{\theta}$ is the set of diagonal matrices $\operatorname{diag}\left(z_{1}, z_{1}^{-1}, z_{1}^{-1}, z_{1}, \ldots, z_{q}, z_{q}^{-1}, z_{q}^{-1}, z_{q}, z_{2 q+1}\right.$, $\left.z_{2 q+1}^{-1}, \ldots, z_{n}, z_{n}^{-1}\right)$ with the $z_{i}$ 's in $\mathbb{T}$. The dominant weights $\left(\lambda_{1}, \ldots, \lambda_{n}\right)$ trivial on $T^{\theta}$ write therefore as partitions of length $q$ with all parts doubled:

$$
\lambda=\left(\lambda_{1}, \lambda_{1}, \ldots, \lambda_{q}, \lambda_{q}, 0, \ldots, 0\right) .
$$

\subsubsection{Special Orthogonal Groups and their Quotients}

Odd and even special orthogonal groups do not have the same kind of root system, and on the other hand, $\mathrm{SO}(n, \mathbb{R})$ is not simply connected and has for fundamental group $\mathbb{Z} / 2 \mathbb{Z}$ for $n \geq 3$. So in theory, the arguments previously recalled apply only for the universal cover $\operatorname{Spin}(n)$. Nonetheless, most of the results will stay true, and in particular the labeling of the irreducible representations; see the end of [3, Chapter 5] for details on this question. In the odd case, a maximal torus in $\operatorname{SO}(2 n+1, \mathbb{R})$ is

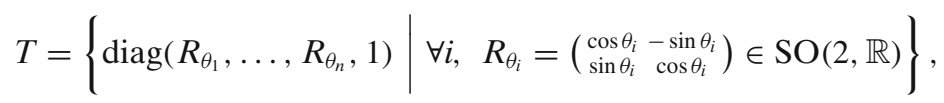

and the Weyl group is again the hyperoctahedral group $\mathfrak{H}_{n}$. The simple roots are $\alpha_{i}=e^{i}-e^{i+1}$ for $i \in \llbracket 1, n-1 \rrbracket$, and $\alpha_{n}=e^{n}$; and the fundamental weights are $\varpi_{i}=$ $e^{1}+\cdots+e^{i}$ for $i \in \llbracket 1, n-1 \rrbracket$, and $\varpi_{n}=\frac{1}{2}\left(e^{1}+\cdots+e^{n}\right)$. Here,

$$
e^{i}\left(\operatorname{diag}\left(\left(\begin{array}{cc}
0 & -a_{1} \\
a_{1} & 0
\end{array}\right), \ldots,\left(\begin{array}{cc}
0 & -a_{n} \\
a_{n} & 0
\end{array}\right), 0\right)\right)=a_{i}
$$

and it corresponds to the morphism $\operatorname{diag}\left(R_{\theta_{1}}, \ldots, R_{\theta_{n}}, 1\right) \mapsto \mathrm{e}^{\mathrm{i} \theta_{i}}$. The dominant weights are then the $\lambda_{1} e^{1}+\cdots+\lambda_{n} e^{n}$, where $\lambda=\left(\lambda_{1} \geq \lambda_{2} \geq \cdots \geq \lambda_{n}\right)$ is either a 
partition of length $n$, or an half-partition of length $n$, where by half-partition we mean a non-increasing sequence of half-integers in $\mathbb{N}^{\prime}=\mathbb{N}+1 / 2$. So, one obtains

$$
\begin{aligned}
D^{\lambda} & =\prod_{1 \leq i<j \leq n} \frac{\lambda_{i}-\lambda_{j}+j-i}{j-i} \prod_{1 \leq i \leq j \leq n} \frac{\lambda_{i}+\lambda_{j}+2 n+1-i-j}{2 n+1-i-j} ; \\
\chi^{\lambda}(k) & =s b_{\lambda}\left(z_{1}, z_{1}^{-1}, \ldots, z_{n}, z_{n}^{-1}, 1\right)=\frac{\operatorname{det}\left(z_{i}^{\lambda_{j}+n-j+1 / 2}-z_{i}^{-\left(\lambda_{j}+n-j+1 / 2\right)}\right)_{1 \leq i, j \leq n}}{\operatorname{det}\left(z_{i}^{n-j+1 / 2}-z_{i}^{-(n-j+1 / 2)}\right)_{1 \leq i, j \leq n}},
\end{aligned}
$$

where $z_{1}^{ \pm 1}, \ldots, z_{n}^{ \pm 1}, 1$ are the eigenvalues of $k$. The Casimir coefficient associated to the highest weight $\lambda$ is $-\kappa_{\lambda}=\frac{1}{2 n+1} \sum_{i=1}^{n} \lambda_{i}^{2}+(2 n+1-2 i) \lambda_{i}$.

In the even case, a maximal torus in $\mathrm{SO}(2 n, \mathbb{R})$ is

$$
T=\left\{\operatorname{diag}\left(R_{\theta_{1}}, \ldots, R_{\theta_{n}}\right) \mid \forall i, \quad R_{\theta_{i}}=\left(\begin{array}{cc}
\cos \theta_{i} & -\sin \theta_{i} \\
\sin \theta_{i} & \cos \theta_{i}
\end{array}\right) \in \mathrm{SO}(2, \mathbb{R})\right\}
$$

and the Weyl group is $\mathfrak{H}_{n}^{+}$, the subgroup of $\mathfrak{H}_{n}$ of index 2 consisting in signed permutations with an even number of signs -1 . The simple roots are $\alpha_{i}=e^{i}-e^{i+1}$ for $i \in \llbracket 1, n-1 \rrbracket$ and $\alpha_{n}=e^{n-1}+e^{n}$; and the fundamental weights are $\varpi_{i}=e^{1}+\cdots+e^{i}$ for $i \in \llbracket 1, n-2 \rrbracket$ and $\varpi_{n-1, n}=\frac{1}{2}\left(e^{1}+\cdots+e^{n-1} \pm e^{n}\right)$. The dominant weights are then $\lambda_{1} e^{1}+\cdots+\lambda_{n-1} e^{n-1}+\varepsilon \lambda_{n} e^{n}$, where $\varepsilon$ is a sign and $\left(\lambda_{1} \geq \cdots \geq \lambda_{n}\right)$ is either a partition or an half-partition of length $n$. So,

$$
\begin{aligned}
D^{\lambda} & =\prod_{1 \leq i<j \leq n} \frac{\lambda_{i}-\lambda_{j}+j-i}{j-i} \frac{\lambda_{i}+\lambda_{j}+2 n-i-j}{2 n-i-j}, \\
\chi^{\lambda}(k) & =s d_{\lambda}\left(z_{1}, z_{1}^{-1}, \ldots, z_{n}, z_{n}^{-1}\right) \\
& =\frac{\operatorname{det}\left(z_{i}^{\lambda_{j}+n-j}-z_{i}^{-\left(\lambda_{j}+n-j\right)}\right)_{1 \leq i, j \leq n}+\operatorname{det}\left(z_{i}^{\lambda_{j}+n-j}+z_{i}^{-\left(\lambda_{j}+n-j\right)}\right)_{1 \leq i, j \leq n}}{\operatorname{det}\left(z_{i}^{n-j}+z_{i}^{-(n-j)}\right)_{1 \leq i, j \leq n}},
\end{aligned}
$$

and $-\kappa_{\lambda}=\frac{1}{2 n} \sum_{i=1}^{n} \lambda_{i}^{2}+(2 n-2 i) \lambda_{i}$.

For real Grassmannian varieties $\mathrm{SO}(n, \mathbb{R}) /(\mathrm{SO}(n-q, \mathbb{R}) \times \mathrm{SO}(q, \mathbb{R}))$ and for spaces of complex structures $\operatorname{SO}(2 n, \mathbb{R}) / \mathrm{U}(n, \mathbb{C})$, one cannot directly apply the Cartan-Helgason theorem, since $\mathrm{SO}(n, \mathbb{R})$ is not simply connected. A rigorous way to deal with this problem is to first look at quotients of the spin group $\operatorname{Spin}(n)$. For instance, consider the Grassmannian variety of non-oriented vector spaces $\operatorname{Gr}^{ \pm}(n, q, \mathbb{R}) \simeq \operatorname{Spin}(n) /(\operatorname{Spin}(n-q) \times \operatorname{Spin}(q)) ; \operatorname{Gr}(n, q, \mathbb{R})$ is a 2-fold covering of $\mathrm{Gr}^{ \pm}(n, q, \mathbb{R})$. The defining map of $\mathrm{Gr}^{ \pm}(n, q, \mathbb{R})$ corresponds to the involution of $\mathrm{SO}(n, \mathbb{R})$ given by $\theta(g)=N_{n, q} g N_{n, q}$, where

$$
N_{n, q}=\left(\begin{array}{cccc}
T_{2} & & & \\
& \ddots & & \\
& & T_{2} & \\
& & & I_{n-2 q}
\end{array}\right)
$$

with $q$ blocks $T_{2}$. Then $T^{\theta}$ is $(\mathbb{Z} / 2 \mathbb{Z})^{q} \times(\mathrm{SO}(2, \mathbb{R}))^{\left\lfloor\frac{n}{2}\right\rfloor-q}$, so the dominant weights trivial on $T^{\theta}$ write as $\lambda=\left(\lambda_{1}, \ldots, \lambda_{q}, 0, \ldots, 0\right)$, with $\lambda_{i} \equiv 0 \bmod 2$ for all $i \in \llbracket 1, q \rrbracket$. 
They are therefore given by an integer partition of length $q$, with all parts even. Now, for the simply connected Grassmannian variety $\operatorname{Gr}(n, q, \mathbb{R})$, there are twice as many spherical representations, as $T^{\theta}$ is in this case isomorphic to $\left((\mathbb{Z} / 2 \mathbb{Z})^{q} /(\mathbb{Z} / 2 \mathbb{Z})\right) \times$ $\mathbb{T}^{\left\lfloor\frac{n}{2}\right\rfloor-q}$, instead of $(\mathbb{Z} / 2 \mathbb{Z})^{q} \times \mathbb{T}^{\left\lfloor\frac{n}{2}\right\rfloor-q}$. Therefore, the condition of parity is now

$$
\forall i, j \in \llbracket 1, q \rrbracket, \quad \lambda_{i} \equiv \lambda_{j} \bmod 2
$$

Similar considerations show that for the spaces $\mathrm{SO}(2 n, \mathbb{R}) / \mathrm{U}(n, \mathbb{C})$, the dominant weights $\lambda$ trivial on the intersection $T^{\theta}$ are given by

$$
\lambda=\left(\lambda_{1}, \lambda_{1}, \ldots, \lambda_{m}, \lambda_{m}\right) \text { or } \lambda=\left(\lambda_{1}, \lambda_{1}, \ldots, \lambda_{m}, \lambda_{m}, 0\right)
$$

that is to say a partition with all non-zero parts that are doubled.

\subsubsection{Summary}

Let us summarize the previous results (this is redundant, but very useful in order to follow all the computations of Section 3). We denote: $\mathfrak{Y}_{n}$ the set of partitions of length $n ; \mathfrak{Z}_{n}$ the set of non-decreasing sequences of (possibly negative) integers; $\frac{1}{2} \mathfrak{Y}_{n}$ the set of partitions and half-partitions of length $n ; 2 \mathfrak{Y}_{n}$ the set of partitions of length $n$ with even parts; $2 \mathfrak{Y}_{n} \boxplus 1$ the set of partitions of length $n$ with odd parts; and $\mathfrak{Y} \mathfrak{Y}_{n}$ the set of partitions of length $n$ and with all non-zero parts doubled. It is understood that if $i$ is too big, then $\lambda_{i}=0$ for a partition or an half-partition $\lambda$ of prescribed length.

Theorem 11 The density of the law $\mu_{t}$ of the Brownian motion traced on a classical simple compact Lie group writes as:

$$
\begin{aligned}
& \sum_{\lambda \in \mathfrak{Y}_{n-1}} \mathrm{e}^{-\frac{t}{2 n}\left(-\frac{|\lambda|^{2}}{n}+\sum_{i=1}^{n-1} \lambda_{i}^{2}+(n+1-2 i) \lambda_{i}\right)}\left(\prod_{1 \leq i<j \leq n} \frac{\lambda_{i}-\lambda_{j}+j-i}{j-i}\right) s_{\lambda}(k) ; \\
& \sum_{\lambda \in \mathfrak{Z}_{n}} \mathrm{e}^{-\frac{t}{2 n} \sum_{i=1}^{n} \lambda_{i}^{2}+(n+1-2 i) \lambda_{i}}\left(\prod_{1 \leq i<j \leq n} \frac{\lambda_{i}-\lambda_{j}+j-i}{j-i}\right) s_{\lambda}(k) ; \\
& \sum_{\lambda \in \mathfrak{Y}_{n}} \mathrm{e}^{-\frac{t}{4 n} \sum_{i=1}^{n} \lambda_{i}^{2}+(2 n+2-2 i) \lambda_{i}}\left(\prod_{1 \leq i<j \leq n} \frac{\lambda_{i}-\lambda_{j}+j-i}{j-i} \prod_{1 \leq i \leq j \leq n} \frac{\lambda_{i}+\lambda_{j}+2 n+2-i-j}{2 n+2-i-j}\right) s c_{\lambda}(k) ; \\
& \sum_{\lambda \in \frac{1}{2} \mathfrak{Y}_{n}} \mathrm{e}^{-\frac{t}{4 n+2} \sum_{i=1}^{n} \lambda_{i}^{2}+(2 n+1-2 i) \lambda_{i}}\left(\prod_{1 \leq i<j \leq n} \frac{\lambda_{i}-\lambda_{j}+j-i}{j-i} \prod_{1 \leq i \leq j \leq n} \frac{\lambda_{i}+\lambda_{j}+2 n+1-i-j}{2 n+1-i-j}\right) s b_{\lambda}(k) ; \\
& \sum_{\lambda \in \frac{1}{2} \mathfrak{Y}_{n}} \mathrm{e}^{-\frac{t}{4 n} \sum_{i=1}^{n} \lambda_{i}^{2}+(2 n-2 i) \lambda_{i}}\left(\prod_{1 \leq i<j \leq n} \frac{\left(\lambda_{i}-\lambda_{j}+j-i\right)\left(\lambda_{i}+\lambda_{j}+2 n-i-j\right)}{(j-i)(2 n-i-j)}\right)\left(s d_{\lambda}(k)+s d_{\varepsilon \lambda}(k)\right)
\end{aligned}
$$

respectively for special unitary groups $\mathrm{SU}(n, \mathbb{C})$, unitary groups $\mathrm{U}(n, \mathbb{C})$, symplectic groups $\mathrm{USp}(n, \mathbb{H})$, odd special orthogonal groups $\mathrm{SO}(2 n+1, \mathbb{R})$, and even special 
orthogonal groups $\mathrm{SO}(2 n, \mathbb{R})$. In this last case, $\varepsilon \lambda=\left(\lambda_{1}, \ldots, \lambda_{n-1},-\lambda_{n}\right)$, and it is agreed that $s d_{\lambda}+s d_{\varepsilon \lambda}$ stands for $s d_{\lambda}$ if $\lambda_{n}=0$.

We denote generically $\phi_{\lambda}(x)$ a zonal spherical function associated to a spherical representation (the function depends of course of the implicit type of the space considered). The density of the law $\mu_{t}$ of the Brownian motion traced on a classical simple compact symmetric space writes then as follows:

$$
\begin{aligned}
& \sum_{\lambda \in 2 \mathfrak{Y}_{n-1}} \mathrm{e}^{-\frac{t}{2 n}\left(-\frac{|\lambda|^{2}}{n}+\sum_{i=1}^{n-1} \lambda_{i}^{2}+(n+1-2 i) \lambda_{i}\right)}\left(\prod_{1 \leq i<j \leq n} \frac{\lambda_{i}-\lambda_{j}+j-i}{j-i}\right) \phi_{\lambda}(x) ; \\
& \sum_{\lambda \in \mathfrak{Y Y Y}_{2 n-1}} \mathrm{e}^{-\frac{t}{4 n}\left(-\frac{|\lambda|^{2}}{2 n}+\sum_{i=1}^{2 n-2} \lambda_{i}^{2}+(2 n+1-2 i) \lambda_{i}\right)}\left(\prod_{1 \leq i<j \leq 2 n} \frac{\lambda_{i}-\lambda_{j}+j-i}{j-i}\right) \phi_{\lambda}(x)
\end{aligned}
$$$$
\sum_{\lambda \in \mathfrak{Y}_{q}} \mathrm{e}^{-\frac{t}{n} \sum_{i=1}^{q} \lambda_{i}^{2}+(n+1-2 i) \lambda_{i}}\left(\prod_{1 \leq i<j \leq n} \frac{\lambda_{i}-\lambda_{j}+j-i}{j-i}\right) \phi_{\lambda}(x)
$$$$
\sum_{\lambda \in 2 \mathfrak{Y}_{n}} \mathrm{e}^{-\frac{t}{4 n} \sum_{i=1}^{n} \lambda_{i}^{2}+(2 n+2-2 i) \lambda_{i}}\left(\prod_{1 \leq i<j \leq n} \frac{\lambda_{i}-\lambda_{j}+j-i}{j-i} \prod_{1 \leq i \leq j \leq n} \frac{\lambda_{i}+\lambda_{j}+2 n+2-i-j}{2 n+2-i-j}\right) \phi_{\lambda}(x)
$$$$
\sum_{\lambda \in \mathfrak{Y} \mathfrak{Y}_{2 q}} \mathrm{e}^{-\frac{t}{4 n} \sum_{i=1}^{2 q} \lambda_{i}^{2}+(2 n+2-2 i) \lambda_{i}}\left(\prod_{1 \leq i<j \leq n} \frac{\lambda_{i}-\lambda_{j}+j-i}{j-i} \prod_{1 \leq i \leq j \leq n} \frac{\lambda_{i}+\lambda_{j}+2 n+2-i-j}{2 n+2-i-j}\right) \phi_{\lambda}(x)
$$$$
\sum_{\lambda \in \mathfrak{Y Y Y}_{n}} \mathrm{e}^{-\frac{t}{4 n} \sum_{i=1}^{n} \lambda_{i}^{2}+(2 n-2 i) \lambda_{i}}\left(\prod_{1 \leq i<j \leq n} \frac{\left(\lambda_{i}-\lambda_{j}+j-i\right)\left(\lambda_{i}+\lambda_{j}+2 n-i-j\right)}{(j-i)(2 n-i-j)}\right) \phi_{\lambda}(x)
$$$$
\sum_{\lambda \in 2 \mathfrak{Y}_{q} \sqcup 2 \mathfrak{Y}_{q} \boxplus 1} \mathrm{e}^{-\frac{t}{4 n+2} \sum_{i=1}^{q} \lambda_{i}^{2}+(2 n+1-2 i) \lambda_{i}}\left(\prod_{1 \leq i<j \leq n} \frac{\lambda_{i}-\lambda_{j}+j-i}{j-i} \prod_{1 \leq i \leq j \leq n} \frac{\lambda_{i}+\lambda_{j}+2 n+1-i-j}{2 n+1-i-j}\right) \phi_{\lambda}(x)
$$$$
\sum_{\lambda \in 2 \mathfrak{Y}_{q} \sqcup 2 \mathfrak{Y}_{q} \boxplus 1} \mathrm{e}^{-\frac{t}{4 n} \sum_{i=1}^{q} \lambda_{i}^{2}+(2 n-2 i) \lambda_{i}}\left(\prod_{1 \leq i<j \leq n} \frac{\left(\lambda_{i}-\lambda_{j}+j-i\right)\left(\lambda_{i}+\lambda_{j}+2 n-i-j\right)}{(j-i)(2 n-i-j)}\right) \phi_{\lambda}(x)
$$

for real structures $\mathrm{SU}(n, \mathbb{C}) / \mathrm{SO}(n, \mathbb{R})$, quaternionic structures $\mathrm{SU}(2 n, \mathbb{C}) / \mathrm{USp}(n, \mathbb{H})$, complex Grassmannian varieties $\operatorname{Gr}(n, q, \mathbb{C})$, complex structures $\operatorname{USp}(n, \mathbb{H}) / \mathrm{U}(n, \mathbb{C})$, quaternionic Grassmannian varieties $\operatorname{Gr}(n, q, \mathbb{H})$, complex structures $\operatorname{SO}(2 n, \mathbb{R}) / \mathrm{U}(n$, $\mathbb{C})$, odd real Grassmannian varieties $\operatorname{Gr}(2 n+1, q, \mathbb{R})$ and even real Grassmannian varieties $\operatorname{Gr}(2 n, q, \mathbb{R})$. 
Remark In the case of complex Grassmannian varieties, it is understood that $\lambda_{n+1-i}=-\lambda_{i}$ as explained before. We have not tried to reduce the expressions in the previous formulas, so some simplifications can be made by replacing the indexing sets of type $2 \mathfrak{Y}_{p}$ or $\mathfrak{Y} \mathfrak{Y}_{p}$ by $\mathfrak{Y}_{p}$. On the other hand, it should be noticed that in each case, the "degree of freedom" in the choice of partitions labeling the irreducible or spherical representations is exactly the rank of the Riemannian variety under consideration, that is to say the maximal dimension of flat totally geodesic submanifolds.

Example (Brownian motions on spheres and projective spaces) Let us examine the case $q=1$ for Grassmannian varieties: it corresponds to real spheres $\mathbb{S}^{n}(\mathbb{R})=\mathrm{SO}(n+1, \mathbb{R}) / \mathrm{SO}(n, \mathbb{R})$, to complex projective spaces $\mathbb{P}^{n}(\mathbb{C})=\mathrm{SU}(n+$ $1, \mathbb{C}) / \mathrm{S}(\mathrm{U}(n, \mathbb{C}) \times \mathrm{U}(1, \mathbb{C}))$ and to quaternionic projective spaces $\mathbb{P}^{n}(\mathbb{H})=\mathrm{USp}(n+$ $1, \mathbb{H}) /(\mathrm{USp}(n, \mathbb{H}) \times \operatorname{USp}(1, \mathbb{H}))$. In each case, spherical representations are labelled by a single integer $k \in \mathbb{N}$. So, the densities are:

$$
\begin{aligned}
& p_{t}^{\mathbb{S}^{n}(\mathbb{R})}(x)=\sum_{k=0}^{\infty} \mathrm{e}^{-\frac{k(k+n-1) t}{2 n+2}} \frac{(n-2+k) !}{(n-1) ! k !}(2 k+n-1) \phi_{n, k}^{\mathbb{R}}(x) ; \\
& p_{t}^{\mathbb{P}^{n}(\mathbb{C})}(x)=\sum_{k=0}^{\infty} \mathrm{e}^{-\frac{k(k+n) t}{n+1}} \frac{((n-1+k) !)^{2}}{(n-1) ! n !(k !)^{2}}(2 k+n) \phi_{n, k}^{\mathbb{C}}(x) ; \\
& p_{t}^{\mathbb{P}^{n}(\mathbb{H})}(x)=\sum_{k=0}^{\infty} \mathrm{e}^{-\frac{k(k+2 n+1) t}{2(n+1)}} \frac{(2 n+k) !(2 n-1+k) !}{(2 n+1) !(2 n-1) !(k+1) ! k !}(2 k+2 n+1) \phi_{n, k}^{\mathbb{H}}(x) .
\end{aligned}
$$

In particular, one recovers the well-known fact that, up to the aforementioned normalization factor $(n+1)$, the eigenvalues of the Laplacian on the $n$-sphere are the integers $k(k+n-1)$, each with multiplicity

$$
\frac{(n-2+k) !}{(n-1) ! k !}(2 k+n-1)
$$

see e.g. [25, Section 3.3].

Example (Torus and Fourier analysis) Take the circle $\mathbb{T}=\mathrm{U}(1, \mathbb{C})=\mathbb{S}^{1}(\mathbb{R})$. The Brownian motion on $\mathbb{T}$ is the projection of the real Brownian motion of density $p_{t}^{\mathbb{R}}(\theta)=\frac{1}{\sqrt{2 \pi t}} \mathrm{e}^{-\theta^{2} / 2 t}$ by the map $\theta \mapsto \mathrm{e}^{\mathrm{i} \theta}$. Thus,

$$
p_{t}^{\mathbb{T}}\left(\mathrm{e}^{\mathrm{i} \theta}\right)=2 \pi \sum_{m=-\infty}^{\infty} p_{t}^{\mathbb{R}}(\theta+2 m \pi)=\sqrt{\frac{2 \pi}{t}} \sum_{m=-\infty}^{\infty} \mathrm{e}^{-\frac{(\theta+2 m \pi)^{2}}{2 t}}=\sqrt{\frac{2 \pi}{t}} S(\theta, t) .
$$


The series $S(\theta, t)$ is smooth and $2 \pi$-periodic, so it is equal to its Fourier series $\sum_{n=-\infty}^{\infty} c_{k}(S(t)) \mathrm{e}^{k i \theta}$, with

$$
c_{k}(S(t))=\sum_{m=-\infty}^{\infty} \int_{0}^{2 \pi} \mathrm{e}^{-\frac{(\theta+2 m \pi)^{2}}{2 t}} \mathrm{e}^{-k \mathrm{i} \theta} \frac{d \theta}{2 \pi}=\frac{1}{2 \pi} \int_{\mathbb{R}} \mathrm{e}^{-\frac{y^{2}}{2 t}-k \mathrm{i} y} d y=\sqrt{\frac{t}{2 \pi}} \mathrm{e}^{-\frac{k^{2} t}{2}} .
$$

Thus, the density of the Brownian motion on the circle with respect to the Haar measure $\frac{d \theta}{2 \pi}$ is

$$
p_{t}^{\mathbb{T}}\left(\mathrm{e}^{\mathrm{i} \theta}\right)=\sum_{k=-\infty}^{\infty} \mathrm{e}^{-\frac{k^{2} t}{2}} \mathrm{e}^{k \mathrm{i} \theta}=1+2 \sum_{k=1}^{\infty} \mathrm{e}^{-\frac{k^{2} t}{2}} \cos k \theta
$$

Since $s_{(k)}\left(\mathrm{e}^{\mathrm{i} \theta}\right)=\mathrm{e}^{k \mathrm{i} \theta}$, this is indeed a specialization of the second formula of Theorem 11 , for $U(1, \mathbb{C})$.

Example (Brownian motion on the 3-dimensional sphere) Consider the Brownian motion on $\operatorname{USp}(1, \mathbb{H})$, which is also $\operatorname{SU}(2, \mathbb{C})$ by one of the exceptional isomorphisms. The specialization of the first formula of Theorem 11 for $\mathrm{SU}(2, \mathbb{C})$ gives

$$
p_{t}^{\mathrm{SU}(2, \mathbb{C})}(g)=\sum_{k=0}^{\infty} \mathrm{e}^{-\frac{k(k+2) t}{8}}(k+1) \frac{\sin (k+1) \theta}{\sin \theta},
$$

if $\mathrm{e}^{ \pm \mathrm{i} \theta}$ are the eigenvalues of $g \in \mathrm{SU}(2, \mathbb{C})$. It agrees with the example of $[19$, Section 4], and also with formula 2.8 when $n=3$, since the group of unit quaternions is topologically a 3 -sphere.

Remark The previous examples show that the restrictions $n \geq n_{0}$ are not entirely necessary for the formulas of Theorem 11 to hold. One should only beware that the root systems of type $\mathrm{B}_{1}, \mathrm{C}_{1}, \mathrm{D}_{1}$ and $\mathrm{D}_{2}$ are somewhat degenerated, and that the dominant weights do not have the same indexing set as for $\mathrm{B}_{n \geq 2}$ or $\mathrm{C}_{n \geq 2}$ or $\mathrm{D}_{n \geq 3}$. For instance, for the special orthogonal group $\operatorname{SO}(3, \mathbb{R})$, the only positive root is $e^{1}$, and the only fundamental weight is also $e^{1}$. Consequently, irreducible representations have highest weights $k e^{1}$ with $k \in \mathbb{N}$; the dimension of the representation of label $k$ is $2 k+1$, and the corresponding character is again $\frac{\sin (k+1) \theta}{\sin \theta}$ if $\mathrm{e}^{\mathrm{i} \theta}$ and $\mathrm{e}^{-\mathrm{i} \theta}$ are the non-trivial eigenvalues of the considered rotation. So

$$
p_{t}^{\mathrm{SO}(3, \mathbb{R})}(g)=\sum_{k=0}^{\infty} \mathrm{e}^{-\frac{k(k+1) t}{3}}(2 k+1) \frac{\sin (k+1) \theta}{\sin \theta}
$$

if $g$ is a rotation of angle $\theta$ around some axis.

\section{Upper Bounds after the Cut-off Time}

Let $\mu$ be a probability measure on a compact Lie group $K$ or compact symmetric space $G / K$, that is absolutely continuous with respect to the Haar measure $\eta$, and with density $p$. Cauchy-Schwarz inequality ensures that

$$
4 d_{\mathrm{TV}}(\mu, \eta)^{2}=\left(\int_{X}|p(x)-1| d x\right)^{2} \leq \int_{X}|p(x)-1|^{2} d x=\|p-1\|_{\mathscr{L}^{2}(X)}^{2} .
$$


The discussion of Section 2 allows now to relate the right-hand side of this inequality with the harmonic analysis on $X$. Let us first treat the case of a compact Lie group $K$. If one assumes that $p$ is invariant by conjugacy, then Parseval's identity (Eq. 2.2) shows that the right-hand side is $\sum_{\lambda \in \widehat{K}}\left|\chi^{\lambda}(p-1)\right|^{2}$. However, by orthogonality of characters, for any non-trivial irreducible representation of $K$-i.e., not equal to $\mathbf{1}_{K}$ : $k \in K \mapsto 1$-one has

$$
\chi^{\lambda}(1)=\int_{K} \chi^{\lambda}(k) d k=\int_{K} \chi^{\lambda}(k) \chi^{\mathbf{1}_{K}}\left(k^{-1}\right) d k=0 .
$$

On the other hand, for any measure $\mu$ on the group, $\chi^{\mathbf{1}_{K}}(\mu)=\int_{K} \chi^{\mathbf{1}}(k) \mu(d k)=$ $\int_{K} \mu(d k)=1$. Hence, the inequality now takes the form

$$
4 d_{\mathrm{TV}}\left(\mu, \eta_{K}\right)^{2} \leq \sum_{\lambda \in \widehat{K}}^{\prime}\left|\chi^{\lambda}(p)\right|^{2}
$$

where the ' indicates that we remove the trivial representation from the summation. Similarly, on a compact symmetric space $G / K$, supposing that $p$ is $K$-invariant, Parseval's identity reads $\|p-1\|_{\mathscr{L}^{2}(G / K)}^{2}=\sum_{\lambda \in \widehat{G}^{K}} D^{\lambda}\left|\phi^{\lambda}(p-1)\right|^{2}$. However, for any non-trivial representation $\lambda$,

$$
\phi^{\lambda}(1)=\left\langle e^{\lambda} \mid \int_{G} \rho^{\lambda}(g)\left(e^{\lambda}\right) d g\right\rangle=0 .
$$

Indeed, using only elementary properties of the Haar measure, one sees that $\widehat{1}(\lambda)=$ $\int_{G} \rho^{\lambda}(g) d g=0$, because it is a projector and it has trace $\chi^{\lambda}(1)=0$. So again, the previous inequality can be simplified and it becomes

$$
4 d_{\mathrm{TV}}\left(\mu, \eta_{G / K}\right)^{2} \leq \sum_{\lambda \in \widehat{G}^{K}}^{\prime} D^{\lambda}\left|\phi^{\lambda}(p)\right|^{2} .
$$

In the setting and with the notations of Proposition 10, a bound at time $t$ on $4 d_{\mathrm{TV}}\left(\mu_{t}, \eta_{K}\right)^{2}$ (respectively, on $\left.4 d_{\mathrm{TV}}\left(\mu_{t}, \eta_{G / K}\right)^{2}\right)$ is then

$$
\sum_{\lambda \in \widehat{K}}^{\prime} \mathrm{e}^{-t\langle\lambda+2 \rho \mid \lambda\rangle}\left(D^{\lambda}\right)^{2} \quad ; \quad \text { respectively, } \sum_{\lambda \in \widehat{G}^{K}}^{\prime} \mathrm{e}^{-t\langle\lambda+2 \rho \mid \lambda\rangle} D^{\lambda} .
$$

Proposition 12 In every classical case, $4 d_{\mathrm{TV}}\left(\mu_{t}, \mathrm{Haar}\right)^{2}$ is bounded by $\sum_{\lambda \in W_{n}}^{\prime} A_{n}(\lambda) \mathrm{e}^{-t B_{n}(\lambda)}$, where the indexing sets $W_{n}$ and the constants $B_{n}(\lambda)$ are the same as in Theorem 11, and $A_{n}(\lambda)=\left(D^{\lambda}\right)^{2}$ for compact Lie groups and $D^{\lambda}$ for compact symmetric spaces.

This section is now organized as follows. In Section 3.1, we compute the weights that minimize $B_{n}(\lambda)$; they will give the correct order of decay of the whole series 
after cut-off time. In Section 3.2, we then show case-by-case that all the other terms of the series $S_{n}(t)$ of Proposition 12 can be controlled uniformly. Essentially, we adapt the arguments of [22-24], though we also introduce new computational tricks. As explained in the introduction, the main reason why one has a good control over $S_{n}(t)$ after cut-off time is that each term $T_{n}(\lambda, t)=A_{n}(\lambda) \mathrm{e}^{-t B_{n}(\lambda)}$ of the series $S_{n}(t)$ stays bounded when $t=t_{\text {cut-off }}$, for every $n$, every class $\lambda$ and in every case. We have unfortunately not found a way to factorize all the computations needed to prove this, so each case will have to be treated separately. However, the scheme of the proof will always be the same, and the reader will find the main arguments in Section 3.2.1 (for symplectic groups and their quotients), so he can safely skip Sections 3.2.23.2.4 if he does not want to see the minor modifications needed to treat the other cases.

\subsection{Guessing the Order of Decay of the Dominating Series}

Remember the restriction $n \geq 2$ (respectively, $n \geq 3$ and $n \geq 10$ ) when studying special unitary groups (resp., compact symplectic groups and special orthogonal groups) and their quotients. We use the superscript $\star$ to denote a set of partitions or half-partitions minus the trivial partition $(0,0, \ldots, 0)$. The lemma hereafter allows to guess the correct order of decay of the series under study.

Lemma 13 Each weight $\lambda_{\min }$ indicated in the table hereafter corresponds to an irreducible representation in the case of compact groups, and to a spherical irreducible representation in the case of symmetric spaces of type non-group. The table also gives the corresponding values of $A_{n}$ and $B_{n}$. In the group case, $B_{n}\left(\lambda_{\min }\right)$ is minimal among $\left\{B_{n}(\lambda), \lambda \in W_{n}^{\star}\right\}$.

Remark For symmetric spaces of type non-group, one can also check the minimality of $B_{n}\left(\lambda_{\min }\right)$, except for certain real $\operatorname{Grassmannian}$ varieties $\operatorname{Gr}(n, q, \mathbb{R})$. For instance, if $q=1$, then (1) $q$ labels the geometric representation of $\operatorname{SO}(n, \mathbb{R})$ on $\mathbb{C}^{n}$, which has indeed an invariant vector by $\mathrm{SO}(n-1, \mathbb{R}) \times \mathrm{SO}(1, \mathbb{R})$; and the corresponding value of $B(\lambda)$ is $(n-1) / n<2$. Fortunately, $\lambda_{\min }$, though not minimal, will still yield in this case the correct order of decay of the series $S(t)$.

Remark To each "minimal" weight $\lambda_{\min }$ corresponds a very natural representation. Namely, for a special orthogonal group $\mathrm{SO}(n, \mathbb{R})$ (respectively, a compact symplectic group $\operatorname{USp}(n, \mathbb{H}))$, the minimizer is the "geometric" representation over $\mathbb{C}^{n}$ (respectively $\left.\mathbb{C}^{2 n}\right)$ corresponding to the embedding $\mathrm{SO}(n, \mathbb{R}) \hookrightarrow \operatorname{SO}(n, \mathbb{C}) \hookrightarrow \operatorname{GL}(n, \mathbb{C})$ (respectively $\operatorname{USp}(n, \mathbb{H}) \hookrightarrow \mathrm{SU}(2 n, \mathbb{C}) \hookrightarrow \mathrm{GL}(2 n, \mathbb{C}))$. For a special unitary group $\operatorname{SU}(n, \mathbb{C})$, one has again the geometric representation over $\mathbb{C}^{n}$, and its compose with the involution $k \mapsto\left(k^{t}\right)^{-1}$ corresponds to the label $(1, \ldots, 1)_{n-1}$, which also minimizes $B_{n}(\lambda)$. The case of spherical minimizers is more involved but still workable: we shall detail it in Section 4. 
Proof To avoid any ambiguity, we shall use indices to precise the length of a partition or half-partition. Let us first find the minimizers of $B_{n}(\lambda)$ in the group case:

\begin{tabular}{cccc}
\hline$K$ or $G / K$ & $\lambda_{\min }$ & $B_{n}\left(\lambda_{\min }\right)$ & $A_{n}\left(\lambda_{\min }\right)$ \\
\hline $\mathrm{SO}(2 n+1, \mathbb{R})$ & $(1,0, \ldots, 0)_{n}$ & $\frac{2 n}{2 n+1}$ & $(2 n+1)^{2}$ \\
$\mathrm{SO}(2 n, \mathbb{R})$ & $(1,0, \ldots, 0)_{n}$ & $\frac{2 n-1}{2 n}$ & $4 n^{2}$ \\
$\mathrm{SU}(n, \mathbb{C})$ & $(1,0, \ldots, 0)_{n-1}$ & $1-\frac{1}{n^{2}}$ & $n^{2}$ \\
$\mathrm{USp}(n, \mathbb{H})$ & $(1,0, \ldots, 0)_{n}$ & $\frac{2 n+1}{2 n}$ & $4 n^{2}$ \\
$\mathrm{Gr}(2 n+1, q, \mathbb{R})$ & $(2,0, \ldots, 0)_{q}$ & 2 & $2 n^{2}+3 n$ \\
$\operatorname{Gr}(2 n, q, \mathbb{R})$ & $(2,0, \ldots, 0)_{q}$ & 2 & $2 n^{2}+n-1$ \\
$\operatorname{Gr}(n, q, \mathbb{C})$ & $(1,0, \ldots, 0)_{q}$ & 2 & $n^{2}-1$ \\
$\operatorname{Gr}(n, q, \mathbb{H})$ & $(1,1,0, \ldots, 0)_{2 q}$ & 2 & $(n-1)(2 n+1)$ \\
$\mathrm{SO}(2 n, \mathbb{R}) / \mathrm{U}(n, \mathbb{C})$ & $(1,1,0, \ldots, 0)_{n}$ & $\frac{2(n-1)}{n}$ & $n(2 n-1)$ \\
$\mathrm{SU}(n, \mathbb{C}) / \mathrm{SO}(n, \mathbb{R})$ & $(2,0, \ldots, 0)_{n-1}$ & $\frac{2(n-1)(n+2)}{n^{2}}$ & $\frac{n(n+1)}{2}$ \\
$\mathrm{SU}(2 n, \mathbb{C}) / \mathrm{USp}(n, \mathbb{H})$ & $(1,1,0, \ldots, 0)_{2 n-1}$ & $\frac{(n-1)(2 n+1)}{n^{2}}$ & $n(2 n-1)$ \\
$\mathrm{USp}(n, \mathbb{H}) / \mathrm{U}(n, \mathbb{C})$ & $(2,0, \ldots, 0)_{n}$ & $\frac{2(n+1)}{n}$ & $n(2 n+1)$ \\
\hline
\end{tabular}

- $\mathrm{SU}(n)$ : one has to minimize

$$
\begin{aligned}
-\frac{|\lambda|^{2}}{n}+\sum_{i=1}^{n-1} \lambda_{i}^{2}+(n+1-2 i) \lambda_{i}= & \frac{1}{n}\left(\sum_{1 \leq i<j \leq n}\left(\lambda_{i}-\lambda_{j}\right)^{2}\right) \\
& +\left(\sum_{i=1}^{n-1} i(n-i)\left(\lambda_{i}-\lambda_{i+1}\right)\right)=A+B
\end{aligned}
$$

over $\mathfrak{Y}_{n-1}^{\star}$. In $B$, at least one term is non-zero, so

$$
B \geq\left(\min _{i \in \llbracket 1, n-1 \rrbracket} i(n-i)\right)=n-1,
$$

with equality if and only if $\lambda=(1,0, \ldots, 0)_{n-1}$ or $\lambda=(1, \ldots, 1)_{n-1}$. In both cases, $A$ is then equal to $\frac{n-1}{n}$. However, $\frac{n-1}{n}$ is also the minimum value of $A$ over $\mathfrak{Y}_{n-1}^{\star}$. Indeed, there is at least one index $l \in \llbracket 1, n-1 \rrbracket$ such that $\lambda_{l}>\lambda_{l+1}$. Then all the $\left(\lambda_{i}-\lambda_{j}\right)^{2}$ with $i \leq l$ and $j \geq l+1$ give a contribution at least equal to 1 , and there are $l(n-l)$ such contributions. Thus

$$
A \geq \frac{l(n-l)}{n} \geq \frac{n-1}{n},
$$

and one concludes that $\min B_{n}(\lambda)$ is obtained only for the two aforementioned partitions, and is equal to $\frac{1}{n}\left(A_{\min }+B_{\min }\right)=1-\frac{1}{n^{2}}$. 
- $\mathrm{SO}(2 n)$ : the quantity to minimize over $\frac{1}{2} \mathfrak{Y}_{n}^{\star}$ is

$$
\left(\sum_{i=1}^{n} \lambda_{i}^{2}\right)+\left(\sum_{i=1}^{n-2} i(2 n-1-i)\left(\lambda_{i}-\lambda_{i+1}\right)\right)+n(n-1) \lambda_{n-1}=A+B+C,
$$

again with $A, B$ and $C$ non-negative in each case. Only $A$ involves $\lambda_{n}$, so a minimizer satisfies necessarily $\lambda_{n}=0$ (partitions) or $\lambda_{n}=\frac{1}{2}$ (half-partitions). In the case of partitions, a minimizer of $B+C$ is $(1,0, \ldots, 0)_{n}$, which gives the value $\min _{i \in \llbracket 1, n-1 \rrbracket} i(2 n-1-i)=2 n-2$. The same sequence minimizes $A$ over $\mathfrak{Y}_{n}^{\star}$, so the minimal value of $A+B+C$ over non-trivial partitions is $2 n-1$ and it is obtained only for $(1,0, \ldots, 0)_{n}$. On the other hand, over half-partitions, the minimizer is $\left(\frac{1}{2}, \ldots, \frac{1}{2}\right)_{n}$, giving the value

$$
\frac{n}{4}+\frac{n(n-1)}{2}=\frac{n(2 n-1)}{4} .
$$

Since we assume $2 n \geq 10$ and therefore $n \geq 5$, this value is strictly bigger than $2 n-1$, so the only minimizer of $B_{n}(\lambda)$ in $\frac{1}{2} \mathfrak{Y}_{n}^{\star}$ is $(1,0, \ldots, 0)_{n}$.

- $\mathrm{SO}(2 n+1)$ : exactly the same reasoning gives the unique minimizer $(1,0, \ldots, 0)_{n}$, with corresponding value $2 n$ for $A+B+C=(2 n+1) B_{n}(\lambda)$.

- $\operatorname{USp}(n)$ : here one has only to look at partitions, and the same reasoning as for $\mathrm{SO}(2 n)$ and $\mathrm{SO}(2 n+1)$ yields the unique minimizer $(1,0, \ldots, 0)_{n}$, corresponding to the value $2 n+1$ for $2 n B_{n}(\lambda)$.

The spherical minimizers are obtained by the same techniques; however, some cases (with $n$ or $q$ too small) are exceptional, so we have only retained in the statement of our Lemma the "generic" minimizer. The corresponding values of $A_{n}(\lambda)$ and $B_{n}(\lambda)$ are easy calculations.

Suppose for a moment that the series $S_{n}(t)$ of Proposition 12 has the same behavior as its "largest term" $A_{n}\left(\lambda_{\min }\right) \mathrm{e}^{-t B_{n}\left(\lambda_{\min }\right)}$. We shall show in a moment that this is indeed true just after cut-off time (for $n$ big enough). Then, $S_{n}(t)$ is a $O(\cdot)$ of

- $n^{2} \mathrm{e}^{-t}$ for classical simple compact Lie groups;

- $n^{2} \mathrm{e}^{-2 t}$ for classical simple compact symmetric spaces of type non-group.

Set then $t_{n, \varepsilon}=\alpha(1+\varepsilon) \log n$, with $\alpha=2$ in the case $n^{2} \mathrm{e}^{-t}$, and $\alpha=1$ in the case $n^{2} \mathrm{e}^{-2 t}$. Under the assumption $S_{n}(t) \sim A_{n}\left(\lambda_{\min }\right) \mathrm{e}^{-t B_{n}\left(\lambda_{\min }\right)}$, one has $S_{n}\left(t_{n, \varepsilon}\right)=$ $O\left(n^{-2 \varepsilon}\right)$. Thus, the previous computations lead to the following guess: the mixing time is

- $2 \log n$ for classical simple compact Lie groups;

- $\log n$ for classical simple compact symmetric spaces of type non-group.

3.2 Growth of the Dimensions Versus Decay of the Laplace-Beltrami Eigenvalues

The estimate $S_{n}\left(t_{n, \varepsilon}\right) \sim A_{n}\left(\lambda_{\min }\right) \mathrm{e}^{-t_{n, \varepsilon} B_{n}(\lambda)}=O\left(n^{-2 \varepsilon}\right)$ might seem very optimistic; nonetheless, we are going to prove that the sum of all the other terms $A_{n}(\lambda) \mathrm{e}^{-t_{n, \varepsilon} B_{n}(\lambda)}$ 
in $S_{n}(t)$ does not change too much this bound, and that one still has at least $S\left(t_{n, \varepsilon}\right)=$ $O\left(n^{-\frac{\varepsilon}{2}}\right)$. We actually believe that at least in the group case, the exponent $2 \varepsilon$ is good, cf. the remark before Section 3.1-the previous discussion shows that it is then optimal.

Suppose that one can bound $A_{n}(\lambda) \mathrm{e}^{-t_{n, \varepsilon} B_{n}(\lambda)}$ by $c(n)^{|\lambda|}$, where $|\lambda|$ is the size of the partition and $c(n)$ is some function of $n$ that goes to 0 as $n$ goes to infinity (say, $C n^{-\delta \varepsilon}$ ). We can then use:

Lemma 14 Assume $x \leq \frac{1}{2}$. Then, the sum over all partitions $\sum_{\lambda} x^{|\lambda|}$, which is convergent, is smaller than $1+5 x$. Consequently,

$$
\sum_{\substack{\lambda \in \mathfrak{Y}_{n} \\ \lambda \neq(0, \ldots, 0)}} x^{|\lambda|} \leq 5 x
$$

Proof The power series $P(x)=\sum_{\lambda} x^{|\lambda|}=\prod_{i=1}^{\infty} \frac{1}{1-x^{i}}=1+x+2 x^{2}+3 x^{3}+5 x^{4}+\cdots$ has radius of convergence 1 , and it is obviously convex on $\mathbb{R}_{+}$. Thus, it suffices to verify the bound at $x=0$ and $x=\frac{1}{2}$. However,

$$
P(0)=1=1+(5 \times 0) \quad ; \quad P\left(\frac{1}{2}\right) \leq 3.463 \leq 1+\left(5 \times \frac{1}{2}\right) .
$$

With this in mind, the idea is then to control the growth of the coefficients $A_{n}(\lambda)$, starting from the trivial partition $(0, \ldots, 0)$. This is also what is done in $[22,23]$, but the way we make our partitions grow is different. The simplest cases to treat in this perspective are the compact symplectic groups and their quotients.

\subsubsection{Symplectic Groups and their Quotients}

Set $t_{n, \varepsilon}=2(1+\varepsilon) \log n$; in particular, $t_{n, 0}=2 \log n$. We fix a partition $\lambda \in \mathfrak{Y}_{n}$, and for $k \leq \lambda_{n}$, we denote $\rho_{k, n}$ the quotient of the dimensions $D^{\lambda}$ associated to the two rectangular partitions

$$
(k, \ldots, k)_{n} \text { and }(k-1, \ldots, k-1)_{n} \text {. }
$$

Using the formula given in Section 2.3 in the case of compact symplectic groups, one obtains:

$$
\begin{aligned}
\rho_{k, n} & =\prod_{1 \leq i \leq j \leq n} \frac{2 k+2 n+2-i-j}{2 k+2 n-i-j} \\
& =\prod_{1 \leq i \leq j \leq n} 1+\frac{2}{2 k+2 n-i-j} \leq \exp \left(\sum_{1 \leq i \leq j \leq n} \frac{2}{2 k+2 n-i-j}\right) .
\end{aligned}
$$


The double sum can be estimated by standard comparison techniques between sums and integrals. Namely, since $x, y \mapsto \frac{1}{2 k+2 n-x-y}$ is convex on $\{(x, y) \mid x \geq 0, y \geq$ $0,2 k+2 n \geq x+y\}$, one can bound each term by

$$
\frac{2}{2 k+2 n-i-j} \leq \iint_{\left[i-\frac{1}{2}, i+\frac{1}{2}\right] \times\left[j-\frac{1}{2}, j+\frac{1}{2}\right]} \frac{2}{2 k+2 n-x-y} d x d y .
$$

We use this bound for non-diagonal terms with indices $i<j$, and for diagonal terms with $i=j$, we use the simpler bound

$$
\sum_{i=1}^{n} \frac{1}{k+n-i}=\sum_{u=0}^{n-1} \frac{1}{k+u}=H_{k+n-1}-H_{k-1} \leq \frac{1}{k}+\log (k+n-1)-\log k
$$

where $H_{n}$ denotes the $n$-th harmonic sum. So,

$$
\begin{aligned}
\log \rho_{k, n} \leq & \sum_{1 \leq i \leq j \leq n} \frac{2}{2 k+2 n-i-j} \leq H_{k+n-1}-H_{k-1}+\iint_{\left[\frac{1}{2}, n+\frac{1}{2}\right]^{2}} \frac{1}{2 k+2 n-x-y} d x d y \\
\leq & \frac{1}{k}+\log (k+n-1)-\log k \\
& +(2 k+2 n-1) \log (2 k+2 n-1)+(2 k-1) \log (2 k-1) \\
& -2(2 k+n-1) \log (2 k+n-1) .
\end{aligned}
$$

On the other hand, the same transformation on partitions makes $-t_{n, 0} B_{n}(\lambda)$ evolve by $-(2 k+n) \log n$. So, if $\eta_{k, n}^{2}$ is the quotient of the quantities $\left(D^{\lambda}\right)^{2} \mathrm{e}^{-t_{n, 0} B_{n}(\lambda)}$ with $\lambda$ as in Eq. 3.1, then

$$
\begin{aligned}
\log \eta_{k, n} \leq & -\frac{2 k+n}{2} \log n+\frac{1}{k}+\log (k+n-1)-\log k \\
& +(2 k+2 n-1) \log (2 k+2 n-1)+(2 k-1) \log (2 k-1) \\
& -2(2 k+n-1) \log (2 k+n-1) .
\end{aligned}
$$

Suppose $k \geq 2$. Then, one can fix $n \geq 3$ and study the previous expression as a function of $k$. Its derivative is then always negative, so $\log \eta_{k, n} \leq \log \eta_{2, n}$, which is also always negative. From this, one deduces that

$$
D^{\lambda} \mathrm{e}^{-\frac{t_{n, 0}}{2} B_{n}(\lambda)} \leq \eta_{1, n}
$$

for any rectangular partition $\left(\lambda_{n}, \ldots, \lambda_{n}\right)_{n}$; indeed, the left-hand side is the product of the contributions $\eta_{k, n}$ for $k$ in $\llbracket 1, \lambda_{n} \rrbracket$. However, $\eta_{1, n}$ is also smaller than 1 : in this case, the dimension is given by the exact formula

$$
D^{(1, \ldots, 1)_{n}}=\mathrm{Cat}_{n+1}=\frac{1}{n+2}\left(\begin{array}{c}
2 n+2 \\
n+1
\end{array}\right)
$$


so $\eta_{1, n}=\mathrm{Cat}_{n+1} \mathrm{e}^{-\frac{n+2}{2} \log n}$, which can be checked to be smaller than 1 for every $n \geq 3$.

So in fact,

$$
D^{\lambda} \mathrm{e}^{-\frac{t_{n, 0}}{2} B_{n}(\lambda)} \leq 1
$$

for any rectangular partition $\left(\lambda_{n}, \ldots, \lambda_{n}\right)_{n}$.

The previous discussion hints at the more general result:

Proposition 15 In the case of compact symplectic groups, at cut-off time,

$$
D^{\lambda} \mathrm{e}^{-\frac{t_{n, 0}}{2} B_{n}(\lambda)} \leq \frac{14}{3}
$$

for any integer partition $\lambda$ of length $n$ (not only the rectangular partitions).

Proof We fix $l \in \llbracket 1, n-1 \rrbracket$, and the idea is again to study the quotient $\rho_{k, l}$ of the dimensions associated to the two partitions

$\left(k+\lambda_{l+1}, \ldots, k+\lambda_{l+1}, \lambda_{l+1}, \ldots, \lambda_{n}\right)_{n}$ and $\left(k-1+\lambda_{l+1}, \ldots, k-1+\lambda_{l+1}, \lambda_{l+1}, \ldots, \lambda_{n}\right)_{n}$,

where $k$ is some integer smaller than $\lambda_{l}-\lambda_{l+1}$-in other words, the $n-l$ last parts of our partition have already been constructed, and one adds $k$ to the $l$ first parts, until $k=\lambda_{l}-\lambda_{l+1}$; see Fig. 3 .

The transformation on partitions described by Eq. 3.2 makes the quantity $-t_{n, 0} B_{n}(\lambda)$ change by $-\frac{l\left(2 k^{\prime}+2 n-l\right)}{n} \log n$. We shall prove that this variation plus $\log \rho_{k, l}$ is almost always negative. For convenience, we will treat separately the cases $l=1$ or 2 and the case $l \geq 3$; hence, suppose first that $l \in \llbracket 3, n-1 \rrbracket$. The quotients of Vandermonde determinants can be simplified as follows:

$$
\begin{aligned}
\rho_{k, l}= & \prod_{j=l+1}^{n} \frac{k+j-1+\lambda_{l+1}-\lambda_{j}}{k+j-l-1+\lambda_{l+1}-\lambda_{j}} \frac{k+\lambda_{l+1}+\lambda_{j}+2 n+1-j}{k+\lambda_{l+1}+\lambda_{j}+2 n+1-j-l} \\
& \times \prod_{1 \leq i \leq j \leq l} \frac{2 k+2 \lambda_{l+1}+2 n+2-i-j}{2 k+2 \lambda_{l+1}+2 n-i-j} .
\end{aligned}
$$

Fig. 3 One makes the partitions grow layer by layer, starting from the bottom

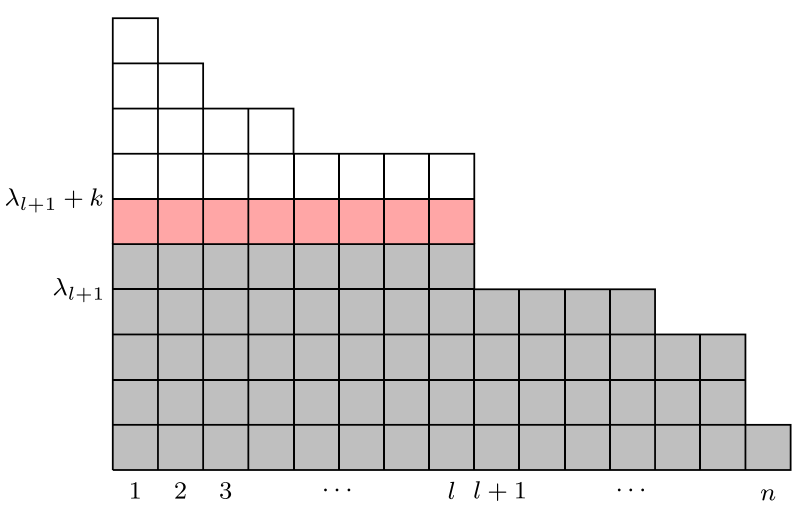


Notice that the second product $\rho_{k, l,(2)}$ in this formula is very similar to $\rho_{k, n}$; the main difference is that indices $i, j$ are now smaller than $l$ (instead of $n)$. Hence, by adapting the arguments, one obtains

$$
\begin{aligned}
\log \rho_{k, l,(2)} \leq & \sum_{1 \leq i \leq j \leq l} \frac{2}{2 k^{\prime}+2 n-i-j} \\
\leq & H_{k^{\prime}+n-1}-H_{k^{\prime}+n-l-1}+\iint_{\left[\frac{1}{2}, l+\frac{1}{2}\right]^{2}} \frac{1}{2 k^{\prime}+2 n-x-y} d x d y \\
\leq & \frac{1}{k^{\prime}+n-l}+\log \left(k^{\prime}+n-1\right)-\log \left(k^{\prime}+n-l\right) \\
& +\left(2 k^{\prime}+2 n-1\right) \log \left(2 k^{\prime}+2 n-1\right) \\
& +\left(2 k^{\prime}+2 n-2 l-1\right) \log \left(2 k^{\prime}+2 n-2 l-1\right) \\
& -2\left(2 k^{\prime}+2 n-l-1\right) \log \left(2 k^{\prime}+2 n-l-1\right)
\end{aligned}
$$

where $k^{\prime}$ stands for $k+\lambda_{l+1}$. So, if $\left(\eta_{k, l}\right)^{2}$ is the quotient of the quantities $\left(D^{\lambda}\right)^{2} \mathrm{e}^{-t_{n, 0} B_{n}(\lambda)}$ with $\lambda$ as in Eq. 3.2, then $\log \eta_{k, l} \leq \log \widetilde{\eta}_{k, l}+\log \rho_{k, l,(1)}$, where $\log \widetilde{\eta}_{k, l}$ is given by

$$
\begin{aligned}
& -\frac{l\left(2 k^{\prime}+2 n-l\right)}{2 n} \log n+\frac{1}{k^{\prime}+n-l}+\log \left(k^{\prime}+n-1\right)-\log \left(k^{\prime}+n-l\right) \\
& +\left(2 k^{\prime}+2 n-1\right) \log \left(2 k^{\prime}+2 n-1\right)+\left(2 k^{\prime}+2 n-2 l-1\right) \log \left(2 k^{\prime}+2 n-2 l-1\right) \\
& -2\left(2 k^{\prime}+2 n-l-1\right) \log \left(2 k^{\prime}+2 n-l-1\right),
\end{aligned}
$$

and $\rho_{k, l,(1)}$ is the first product in the expansion of $\rho_{k, l}$. Let us analyze these two quantities separately.

- $\log \widetilde{\eta}_{k, l}$ : here the technique is really the same as for $\log \eta_{k, n}$. Namely, with $n$ and $l$ fixed, $\log \tilde{\eta}_{k, l}$ appears as a decreasing function of $x=k^{\prime}$, because its derivative with respect to $x$ is

$$
\begin{aligned}
-\frac{l \log n}{n} & -\frac{1}{(x+n-l)^{2}}+\frac{1}{x+n-1}-\frac{1}{x+n-l} \\
& +2(\log (2 x+2 n-1)+\log (2 x+2 n-2 l-1)-2 \log (2 x+2 n-l-1)) .
\end{aligned}
$$

A upper bound on the first line is $-\frac{(l-1) \log n}{n} \leq 0$ (remember that $n \geq 3$ and therefore $\log n \geq 1$ ), and the second line is negative by concavity of the logarithm. From this, one deduces that $\log \widetilde{\eta}_{k, l} \leq \log \widetilde{\eta}_{1, l}$, and we shall use this estimate in order to compensate the other part of $\log \eta_{k, l}$ :

$$
\begin{aligned}
\log \widetilde{\eta}_{k, l} \leq & -\frac{l(2 v+2+2 n-l)}{2 n} \log n+\frac{1}{v+n+1-l}+\log (v+n)-\log (v+n+1-l) \\
& +(2 v+2 n+1) \log (2 v+2 n+1)+(2 v+2 n-2 l+1) \log (2 v+2 n-2 l+1) \\
& -2(2 v+2 n-l+1) \log (2 v+2 n-l+1)
\end{aligned}
$$

where $v$ stands for $\lambda_{l+1}$. 
- $\quad \log \rho_{k, l,(1)}$ : in the product $\rho_{k, l,(1)}$, each term of index $j$ writes as

$$
\begin{aligned}
\frac{\left(k^{\prime}+n\right)^{2}-\left(\lambda_{j}+n+1-j\right)^{2}}{\left(k^{\prime}+n-l\right)^{2}-\left(\lambda_{j}+n+1-j\right)^{2}} & \leq \frac{\left(k^{\prime}+n\right)^{2}-\left(\lambda_{l+1}+n+1-j\right)^{2}}{\left(k^{\prime}+n-l\right)^{2}-\left(\lambda_{l+1}+n+1-j\right)^{2}} \\
& \leq \frac{k+j-1}{k+j-l-1} \frac{k^{\prime \prime}+2 n+1-j}{k^{\prime \prime}+2 n+1-j-l}
\end{aligned}
$$

with $k^{\prime \prime}=k+2 \lambda_{l+1}=k+2 v$; and multiplying all these bounds together, one gets

$$
\rho_{k, l,(1)} \leq \frac{(k+n-1) !}{(k+l-1) !} \frac{(k-1) !}{(k+n-l-1) !} \frac{\left(k^{\prime \prime}+2 n-l\right) !}{\left(k^{\prime \prime}+n\right) !} \frac{\left(k^{\prime \prime}+n-l\right) !}{\left(k^{\prime \prime}+2 n-2 l\right) !} .
$$

Again, this is decreasing in $k$, so

$$
\rho_{k, l,(1)} \leq \frac{n !(2 v+2 n-l+1) !(2 v+n-l+1) !}{l !(n-l) !(2 v+n+1) !(2 v+2 n-2 l+1) !} .
$$

Recall the classical Stirling estimates: for $m \geq 1$,

$\log m !=m \log m+\frac{1}{2} \log m-m+\log \sqrt{2 \pi}+\frac{1}{12 m}-r_{m}, \quad$ with $0 \leq r_{m} \leq \frac{1}{360 m^{3}}$.

It enables us to bound $\log \rho_{k, l,(1)}$ by the sum of the following quantities:

$\star \quad A=(2 v+2 n-l+1) \log (2 v+2 n-l+1)+(2 v+n-l+1) \log (2 v+n-l+1)$

$$
-(2 v+n+1) \log (2 v+n+1)-(2 v+2 n-2 l+1) \log (2 v+2 n-2 l+1) .
$$

$\star \quad B=\frac{1}{2}(\log (2 v+2 n-l+1)+\log (2 v+n-l+1)-\log (2 v+n+1)-\log (2 v+$

$2 n-2 l+1))$, which is non-positive by concavity of the logarithm.

$\star \quad C=n \log n-l \log l-(n-l) \log (n-l)$.

$\star \quad D=\frac{1}{2}(\log n-\log l-\log (n-l))$. This is non-positive unless $n=l+1$ recall that we assume for the moment $l \in \llbracket[3, n-1 \rrbracket$. In that case, it is smaller than $\frac{1}{2(n-1)}$.

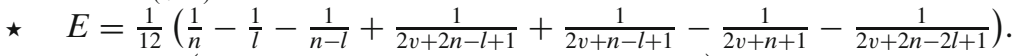

$\star \quad F=\frac{1}{360}\left(\frac{1}{l^{3}}+\frac{1}{(n-l)^{3}}+\frac{1}{(2 v+n+1)^{3}}+\frac{1}{(2 v+2 n-2 l+1)^{3}}\right)$.

The sum of the two last terms $E F=E+F$ happens to be negative. Indeed, $E$ and $F$ are decreasing in $v$ (we use the convexity of $x \mapsto \frac{1}{x^{2}}$ to show that $\frac{d E}{d v} \leq 0$ ), so it suffices to check the result when $v=0$. Then, with $l$ fixed,

$$
\begin{aligned}
E F(n, l)= & \frac{1}{12}\left(\frac{1}{n}-\frac{1}{l}-\frac{1}{n-l}+\frac{1}{2 n-l+1}+\frac{1}{n-l+1}-\frac{1}{n+1}-\frac{1}{2 n-2 l+1}\right) \\
& +\frac{1}{360}\left(\frac{1}{l^{3}}+\frac{1}{(n-l)^{3}}+\frac{1}{(n+1)^{3}}+\frac{1}{(2 n-2 l+1)^{3}}\right)
\end{aligned}
$$

is decreasing in $n$, hence smaller than its value when $n=l+1$. So, it suffices to look at $E F(l+1, l)$, which is now increasing in $l$, but still negative. Thus, in the following, we shall use the bound

$$
\log \rho_{k, l,(1)} \leq A+C+D \leq A+C+\frac{1}{2 n-2} .
$$


Adding together the bounds previously demonstrated, we get

$$
\begin{aligned}
\log \eta_{k, l} \leq & -\frac{l(2 v+2+2 n-l)}{2 n} \log n+\frac{1}{2 n-2}+\frac{1}{v+n+1-l}+\log (v+n)-\log (v+n+1-l) \\
& +(2 v+2 n+1) \log (2 v+2 n+1)-(2 v+2 n-l+1) \log (2 v+2 n-l+1) \\
& +(2 v+n-l+1) \log (2 v+n-l+1)-(2 v+n+1) \log (2 v+n+1) \\
& +n \log n-l \log l-(n-l) \log (n-l) .
\end{aligned}
$$

By concavity of $x \log x$, the sum of the second and third rows is non-positive. What remains is decreasing in $l$ and in $v$, and when $l=3$ and $v=0$, we get

$$
\frac{3}{2 n} \log n+\frac{1}{2 n-2}+\frac{1}{n-2}+\log \left(\frac{n}{n-2}\right)+(n-3) \log \left(\frac{n}{n-3}\right)-3 \log 3
$$

which is maximal for $n=5$, and still (barely) negative at this value. Thus, we have shown so far that $\eta_{k, l} \leq 1$ for any $k$, any $l \in \llbracket 3, n-1 \rrbracket$, and any partition $\lambda$ that we fill as in Fig. 3.

When $l=1$ or $l=2$, the approximations on $\log \eta_{k, l}$ that we were using before are not good enough, but we can treat these cases separately. When $l=1$,

$$
\begin{aligned}
\rho_{k, 1} & =\frac{\lambda_{2}+k+n}{\lambda_{2}+k+n-1} \prod_{j=2}^{n} \frac{k+j-1+\lambda_{2}-\lambda_{j}}{k+j-2+\lambda_{2}-\lambda_{j}} \frac{k+\lambda_{2}+\lambda_{j}+2 n+1-j}{k+\lambda_{2}+\lambda_{j}+2 n-j} \\
& \leq \frac{k+n}{k+n-1} \prod_{j=2}^{n} \frac{k+j-1}{k+j-2} \frac{k+2 n+1-j}{k+2 n-j}=\frac{k+2 n-1}{k} ; \\
\eta_{k, 1} & \leq \frac{k+2 n-1}{k} \mathrm{e}^{-\frac{2 k+2 n-1}{2 n} \log n} .
\end{aligned}
$$

If $k=1$, which only happens once when one makes the partition grow, then the bound above is $2 n \mathrm{e}^{-\frac{2 n+1}{2 n} \log n} \leq 2$. On the other hand, if $k \geq 2$, then the bound is decreasing in $k$ and therefore smaller than $\left(n+\frac{1}{2}\right) \mathrm{e}^{-\frac{2 n+3}{2 n} \log n} \leq 1$. So, one also has $\eta_{k, 1} \leq 1$ for any $k$ but $k=1$, where a correct bound is 2 . Similarly, when $l=2$,

$$
\begin{aligned}
\rho_{k, 2} & =\frac{\lambda_{3}+k+n}{\lambda_{3}+k+n-2} \frac{2 \lambda_{3}+2 k+2 n-1}{2 \lambda_{3}+2 k+2 n-3} \prod_{j=3}^{n} \frac{k+j-1+\lambda_{3}-\lambda_{j}}{k+j-3+\lambda_{3}-\lambda_{j}} \frac{k+\lambda_{3}+\lambda_{j}+2 n+1-j}{k+\lambda_{3}+\lambda_{j}+2 n-1-j} \\
& \leq \frac{k+n}{k+n-2} \frac{2 k+2 n-1}{2 k+2 n-3} \prod_{j=3}^{n} \frac{k+j-1}{k+j-3} \frac{k+2 n+1-j}{k+2 n-1-j}=\frac{k+2 n-2}{k} \frac{k+2 n-1}{k+1} \frac{2 k+2 n-1}{2 k+2 n-3} ; \\
\eta_{k, 2} & \leq \frac{k+2 n-2}{k} \frac{k+2 n-1}{k+1} \frac{2 k+2 n-1}{2 k+2 n-3} \mathrm{e}^{-\frac{2 n+2 k-2}{n} \log n} .
\end{aligned}
$$

Again, the last bound is decreasing in $k$, smaller than $2+\frac{1}{n} \leq \frac{7}{3}$ when $k=1$ and smaller than 1 when $k=2$. Hence, $\eta_{k, 2} \leq 1$ unless $k=1$, where a correct bound is $\frac{7}{3}$ (and again this situation occurs at most once whence making the partition grow). 
Conclusion: every quotient $\eta_{k, l}$ satisfies $\eta_{k, l} \leq 1$, but the two exceptions: $k=1$ and $l=1$ or 2 . The product of the bounds on these two exceptions is $2 \times \frac{7}{3}=\frac{14}{3}$, so for every partition $\lambda$, one has indeed

$$
D^{\lambda} \mathrm{e}^{-\frac{t_{n, 0}}{2} B_{n}(\lambda)}=\prod_{l=1}^{n} \prod_{k=1}^{\lambda_{l}-\lambda_{l+1}} \eta_{k, l} \leq \frac{14}{3}
$$

Remark A small refinement of the previous proof shows that the worst case is in fact the partition $(2,1,0, \ldots, 0)_{n}$-by that we mean that any other partition has quotients $\rho_{k, l}$ that are smaller. Its dimension is provided by the exact formula

$$
D^{\lambda}=\frac{8 n\left(n^{2}-1\right)}{3}
$$

so one can replace the bound $\frac{14}{3}$ of Proposition 15 by $\frac{8}{3}$.

The upper bound (1.7) is now an easy consequence of Lemma 14 and Proposition 15 . For any partition $\lambda$, notice that

$$
B_{n}(\lambda) \geq \frac{1}{2 n} \sum_{i=1}^{n}(2 n+2-2 i) \lambda_{i}=\frac{1}{2 n} \sum_{i=1}^{n} i(2 n+1-i)\left(\lambda_{i}-\lambda_{i+1}\right) \geq \frac{1}{2} \sum_{i=1}^{n} i\left(\lambda_{i}-\lambda_{i+1}\right)=\frac{|\lambda|}{2} .
$$

From this, one deduces that in the case of compact symplectic groups,

$$
S_{n}\left(t_{n, \varepsilon}\right)=\sum_{\lambda \in \mathfrak{Y}_{n}^{\star}}\left(D^{\lambda}\right)^{2} \mathrm{e}^{-t_{n, \varepsilon} B_{n}(\lambda)} \leq \frac{64}{9} \sum_{\lambda \in \mathfrak{Y}_{n}^{\star}} \mathrm{e}^{-\varepsilon|\lambda| \log n} \leq \frac{320}{9 n^{\varepsilon}} \leq \frac{36}{n^{\varepsilon}}
$$

if one assumes that $\frac{1}{n^{\varepsilon}} \leq \frac{1}{2}$ (in order to apply Lemma 14). By Proposition 12, one concludes that

$$
d_{\mathrm{TV}}^{\mathrm{USp}(n, \mathbb{H})}\left(\mu_{2(1+\varepsilon) \log n}, \text { Haar }\right) \leq \frac{3}{n^{\frac{\varepsilon}{2}}} .
$$

Here one can remove the assumption $\frac{1}{n^{\varepsilon}} \leq \frac{1}{2}$ : otherwise, the right-hand side is bigger than 1 and therefore the inequality is trivially satisfied. This ends the proof of the upper bound in the case of compact symplectic groups. For their quotients, one can still use Proposition 15, as follows. For quaternionic Grassmannians,

$$
S_{n}\left(\frac{t_{n, \varepsilon}}{2}\right)=\sum_{\lambda \in \mathfrak{Y} \mathfrak{Y}_{2 q}^{\star}} D^{\lambda} \mathrm{e}^{-\frac{t_{n, \varepsilon}}{2} B_{n}(\lambda)} \leq \frac{8}{3} \sum_{\lambda \in \mathfrak{Y}_{n}^{\star}} \mathrm{e}^{-\frac{\varepsilon}{2}|\lambda| \log n} \leq \frac{40}{3 n^{\frac{\varepsilon}{2}}} \leq \frac{16}{n^{\frac{\varepsilon}{2}}}
$$

assuming $\frac{1}{n^{\frac{e}{2}}} \leq \frac{1}{2}$. This implies that

$$
d_{\mathrm{TV}}^{\mathrm{Gr}(n, q, \mathbb{H})}\left(\mu_{(1+\varepsilon) \log n}, \text { Haar }\right) \leq \frac{2}{n^{\frac{\varepsilon}{4}}} .
$$

Again, the assumption on $n^{\frac{\varepsilon}{2}}$ is superfluous, since otherwise the right-hand side is bigger than 1. Exactly the same proof works for the spaces $\operatorname{USp}(n) / \mathrm{U}(n)$, with the same bound (it may be improved by using the fact that one looks only at even partitions). 


\subsubsection{Odd Special Orthogonal Groups and their Quotients}

Though the same reasoning holds in every case, we unfortunately have to check case by case that everything works. For odd special orthogonal groups $\operatorname{SO}(2 n+1, \mathbb{R})$, set $t_{n, \varepsilon}=2(1+\varepsilon) \log (2 n+1)$, with in particular $t_{n, 0}=2 \log (2 n+1)$. The main difference between $\operatorname{SO}(2 n+1)$ and $\operatorname{USp}(n)$ is the appearance of half-partitions, which is solved by:

Lemma 16 For any integer partition $\lambda$, denote $\lambda \boxplus \frac{1}{2}$ the half-partition $\lambda_{1}+\frac{1}{2}, \lambda_{2}+$ $\frac{1}{2}, \ldots, \lambda_{n}+\frac{1}{2}$.

$$
\frac{D^{\lambda \boxplus \frac{1}{2}}}{D^{\lambda}} \mathrm{e}^{-\frac{t_{n, 0}}{2}\left(B_{n}\left(\lambda \boxplus \frac{1}{2}\right)-B_{n}(\lambda)\right)} \leq \mathrm{e}^{n\left(\log 2-\frac{\log (2 n+1)}{4}\right)} \leq 2 .
$$

Proof The quotient of dimensions is

$$
\prod_{1 \leq i \leq j \leq n} \frac{\lambda_{i}+\lambda_{j}+2 n+2-i-j}{\lambda_{i}+\lambda_{j}+2 n+1-i-j} \leq \prod_{1 \leq i \leq j \leq n} \frac{2 n+2-i-j}{2 n+1-i-j}=2^{n},
$$

and the difference $\frac{t_{n, 0}}{2}\left(B_{n}\left(\lambda \boxplus \frac{1}{2}\right)-B_{n}(\lambda)\right)$ is equal to

$$
\frac{\log (2 n+1)}{2 n+1}\left(\sum_{i=1}^{n} \lambda_{i}+\frac{1}{4}+\frac{2 n+1-2 i}{2}\right) \geq \frac{\log (2 n+1)}{2 n+1}\left(\frac{n}{4}+\frac{n^{2}}{2}\right)=\frac{n \log (2 n+1)}{4}
$$

This yields the first part of the inequality, and the second part is an easy analysis of the variations of the bound with respect to $n$.

Then, for any integer partition $\lambda$, one can as before prove a uniform bound on $D^{\lambda} \mathrm{e}^{-\log (2 n+1) B_{n}(\lambda)}$; the differences are tiny, e.g., in many formulas, $2 n+2$ is replaced by $2 n+1$, or $\frac{1}{2 n}$ is replaced by $\frac{1}{2 n+1}$. We refer to Appendix 5.1 for these computations.

Proposition 17 In the case of odd special orthogonal groups, at cut-off time,

$$
D^{\lambda} \mathrm{e}^{-\frac{t_{n, 0}}{2} B_{n}(\lambda)} \leq \frac{11}{10}
$$

for any integer partition $\lambda$ of length $n$. For half-integer partitions, the bound is replaced by $\frac{11}{5}$.

There is one last computation that needs to be done, namely, the special case $\lambda=$ $\left(\frac{1}{2}, \ldots, \frac{1}{2}\right)_{n}=(0, \ldots, 0)_{n} \boxplus \frac{1}{2}$-it corresponds to the spin representation of $\mathrm{SO}(2 n+$ $1, \mathbb{R})$. The value of $B_{n}(\lambda)$ is then $\frac{n}{4}$, and $D^{\lambda}=2^{n}$. Thus, in this special case,

$$
\left(D^{\lambda}\right)^{2} \mathrm{e}^{-t_{n, \varepsilon} B_{n}(\lambda)} \leq \mathrm{e}^{n \log 4-\frac{n \log (2 n+1)}{2}} \mathrm{e}^{-\frac{\varepsilon n \log (2 n+1)}{2}} \leq \frac{11}{4} \frac{1}{(2 n+1)^{\varepsilon}}
$$


for every $n \geq 5$. On the other hand,

$$
\begin{aligned}
B_{n}(\lambda) & =\frac{1}{2 n+1} \sum_{i=1}^{n} \lambda_{i}^{2}+i(2 n-i)\left(\lambda_{i}-\lambda_{i+1}\right) \\
& \geq \frac{|\lambda|}{2 n+1}+\frac{n}{2 n+1} \sum_{i=1}^{n} i\left(\lambda_{i}-\lambda_{i+1}\right)=\frac{(n+1)|\lambda|}{2 n+1} \geq \frac{|\lambda|}{2},
\end{aligned}
$$

so we can now write:

$$
\begin{aligned}
S_{n}\left(t_{n, \varepsilon}\right) & \leq \frac{11}{4} \frac{1}{(2 n+1)^{\varepsilon}}+\sum_{\lambda \in \mathfrak{Y}_{n}^{\star}}\left(D^{\lambda}\right)^{2} \mathrm{e}^{-t_{n, \varepsilon} B_{n}(\lambda)}+\left(D^{\lambda \boxplus \frac{1}{2}}\right)^{2} \mathrm{e}^{-t_{n, \varepsilon} B_{n}\left(\lambda \boxplus \frac{1}{2}\right)} \\
& \leq \frac{11}{4} \frac{1}{(2 n+1)^{\varepsilon}}+\sum_{\lambda \in \mathfrak{Y}_{n}^{\star}}\left(\left(D^{\lambda}\right)^{2} \mathrm{e}^{-t_{n, 0} B_{n}(\lambda)}+\left(D^{\lambda \boxplus \frac{1}{2}}\right)^{2} \mathrm{e}^{-t_{n, 0} B_{n}\left(\lambda \boxplus \frac{1}{2}\right)}\right) \mathrm{e}^{-2 \varepsilon \log (2 n+1) B_{n}(\lambda)} \\
& \leq \frac{11}{4} \frac{1}{(2 n+1)^{\varepsilon}}+\sum_{\lambda \in \mathfrak{Y}_{n}^{\star}}\left(\frac{121}{100}+\frac{121}{25}\right) \mathrm{e}^{-\varepsilon|\lambda| \log (2 n+1)} \\
& \leq \frac{11}{4} \frac{1}{(2 n+1)^{\varepsilon}}+\frac{121}{20} \sum_{\lambda \in \mathfrak{Y}_{n}^{\star}} \frac{1}{(2 n+1)^{\varepsilon|\lambda|}} \leq \frac{33}{(2 n+1)^{\varepsilon}} \leq \frac{144}{(2 n+1)^{\varepsilon}}
\end{aligned}
$$

if one assumes $\frac{1}{(2 n+1)^{\varepsilon}} \leq \frac{1}{2}$. Thus, by Proposition 12 ,

$$
d_{\mathrm{TV}}^{\mathrm{SO}(2 n+1, \mathbb{R})}\left(\mu_{2(1+\varepsilon) \log (2 n+1)}, \text { Haar }\right) \leq \frac{6}{(2 n+1)^{\frac{\varepsilon}{2}}}
$$

and again we can now remove the assumption $\frac{1}{(2 n+1)^{\varepsilon}} \leq \frac{1}{2}$. The same technique applies to odd real Grassmannians, with

$$
\begin{aligned}
S_{n}\left(\frac{t_{n, \varepsilon}}{2}\right) & =\sum_{\lambda \in\left(2 \mathfrak{Y}_{q} \sqcup 2 \mathfrak{Y}_{q} \boxplus 1\right)^{\star}} D^{\lambda} \mathrm{e}^{-\frac{t_{n, \varepsilon}}{2} B_{n}(\lambda)} \leq \frac{11}{10} \sum_{\lambda \in \mathfrak{Y}_{n}^{\star}} \mathrm{e}^{-\frac{\varepsilon}{2}|\lambda| \log (2 n+1)} \\
& \leq \frac{55}{10(2 n+1)^{\frac{\varepsilon}{2}}} \leq \frac{16}{(2 n+1)^{\frac{\varepsilon}{2}}},
\end{aligned}
$$

and therefore

$$
d_{\mathrm{TV}}^{\mathrm{Gr}(2 n+1, q, \mathbb{R})}\left(\mu_{(1+\varepsilon) \log (2 n+1)}, \text { Haar }\right) \leq \frac{2}{(2 n+1)^{\frac{\varepsilon}{4}}}
$$

\subsubsection{Even Special Orthogonal Groups and their Quotients}

Though the computations have to be done once again, we shall prove exactly the same bounds as before for even special orthogonal groups and even real Grassmannians. Denote $t_{n, \varepsilon}=2(1+\varepsilon) \log (2 n)$. The possibility of a sign \pm for the last part $\lambda_{n}$ of the partitions leads to a coefficient 2 in the series $S_{n}(t)$, and on the other hand, the 
case of half-partitions is reduced to the case of partitions by way of an analogue of Lemma 16. Indeed,

$$
\frac{D^{\lambda \boxplus \frac{1}{2}}}{D^{\lambda}} \mathrm{e}^{-\frac{t_{n, 0}}{2}\left(B_{n}\left(\lambda \boxplus \frac{1}{2}\right)-B_{n}(\lambda)\right)} \leq \mathrm{e}^{n \log 2-\frac{(2 n-1) \log (2 n)}{8}} \leq \frac{12}{5}
$$

for any $n \geq 5$ and any partition. Again, we put the proof of the following Proposition at the end of the paper, in Appendix 5.2.

Proposition 18 In the case of even special orthogonal groups, at cut-off time,

$$
D^{\lambda} \mathrm{e}^{-\frac{t_{n, 0}}{2} B_{n}(\lambda)} \leq \frac{4}{3} \quad\left(\text { respectively, } \frac{48}{15}\right)
$$

for any integer partition (resp. any half-partition) $\lambda$ of length $n$.

Besides, the same proof as in the case of odd special orthogonal groups shows that $B_{n}(\lambda) \geq \frac{|\lambda|}{2}$ for any partition. For the special half-partition $\lambda=(0, \ldots, 0)_{n} \boxplus \frac{1}{2}$ that cannot be treated by combining Lemmas 14 and 16 , one has $D^{\lambda}=2^{n-1}$ and $B_{n}(\lambda)=\frac{n}{4}$, hence

$$
\left(D^{\lambda}\right)^{2} \mathrm{e}^{-t_{n, \varepsilon} B_{n}(\lambda)} \leq \mathrm{e}^{(n-1) \log 4-\frac{n \log (2 n)}{2}} \mathrm{e}^{-\frac{\varepsilon n \log (2 n)}{2}} \leq \frac{1}{(2 n)^{\varepsilon}}
$$

for $n \geq 5$. We conclude that

$$
\begin{aligned}
\frac{1}{2} S_{n}\left(t_{n, \varepsilon}\right) & \leq \frac{1}{(2 n)^{\varepsilon}}+\sum_{\lambda \in \mathfrak{Y}_{n}^{\star}}\left(D^{\lambda}\right)^{2} \mathrm{e}^{-t_{n, \varepsilon} B_{n}(\lambda)}+\left(D^{\lambda \boxplus \frac{1}{2}}\right)^{2} \mathrm{e}^{-t_{n, \varepsilon} B_{n}\left(\lambda \boxplus \frac{1}{2}\right)} \\
& \leq \frac{1}{(2 n)^{\varepsilon}}+\sum_{\lambda \in \mathfrak{Y}_{n}^{\star}}\left(\frac{16}{9}+\frac{2304}{225}\right) \mathrm{e}^{-\varepsilon|\lambda| \log (2 n)} \leq \frac{2749}{45(2 n)^{\varepsilon}} \leq \frac{72}{\left(2 n^{\varepsilon}\right)},
\end{aligned}
$$

and therefore, by Proposition 12,

$$
d_{\mathrm{TV}}^{\mathrm{SO}(2 n, \mathbb{R})}\left(\mu_{2(1+\varepsilon) \log (2 n)}, \text { Haar }\right) \leq \frac{6}{(2 n)^{\frac{\varepsilon}{2}}} .
$$

For even real Grassmannian varieties,

$$
S_{n}\left(\frac{t_{n, \varepsilon}}{2}\right)=\sum_{\lambda \in\left(2 \mathfrak{Y}_{q} \sqcup 2 \mathfrak{Y}_{q} \boxplus 1\right)^{\star}} D^{\lambda} \mathrm{e}^{-\frac{t_{n, \varepsilon}}{2} B_{n}(\lambda)} \leq \frac{4}{3} \sum_{\lambda \in \mathfrak{Y}_{n}^{\star}} \mathrm{e}^{-\frac{\varepsilon}{2}|\lambda| \log (2 n)} \leq \frac{20}{3(2 n)^{\frac{\varepsilon}{2}}} \leq \frac{16}{(2 n)^{\frac{\varepsilon}{2}}},
$$

and again, the total variation distance is bounded by $2 /(2 n)^{\frac{\varepsilon}{4}}$. So, the inequalities take the same form for even and odd special orthogonal groups or real Grassmannians, and the proof of the upper bound in this case is done. The same inequality holds also for the spaces of structures $\mathrm{SO}(2 n) / \mathrm{U}(n)$.

\subsubsection{Special Unitary Groups and their Quotients}

Set $t_{n, \varepsilon}=2(1+\varepsilon) \log n$. For special unitary groups, Weyl's dimension formula fortunately takes a much simpler form than before, but on the other hand, the 
computations on $B_{n}(\lambda)$ are this time a little more subtle. We shall still prove that almost every quotient $\eta_{k, l}$ of the quantities $D^{\lambda} \mathrm{e}^{-t_{n, 0} B_{n}(\lambda)}$ with $\lambda$ going from

$$
\begin{gathered}
\left(\lambda_{l+1}+k-1, \ldots, \lambda_{l+1}+k-1, \lambda_{l+1}, \ldots, \lambda_{n-1}\right)_{n-1} \\
\text { to } \quad\left(\lambda_{l+1}+k, \ldots, \lambda_{l+1}+k, \lambda_{l+1}, \ldots, \lambda_{n-1}\right)_{n-1}
\end{gathered}
$$

is smaller than 1; but in practice, what will happen is that the negative exponentials may be much larger than before, whereas the quotients of dimensions $\rho_{k, l}$ will be much smaller. Consider for a start $\eta_{k, n-1}$. One has

$$
\rho_{k, n-1}=\prod_{i=1}^{n-1} \frac{k+n-i}{k-1+n-i}=\frac{k+n-1}{k},
$$

whereas $B_{n}(\lambda)$ is changed by $\frac{(n-1)(n+2 k-1)}{n^{2}}$. So,

$$
\eta_{k, n-1}=\frac{k+n-1}{k} \mathrm{e}^{-\frac{(n-1)(n+2 k-1)}{n^{2}} \log n} \leq \begin{cases}n \mathrm{e}^{-\frac{n^{2}-1}{n^{2}} \log n}=\mathrm{e}^{\frac{\log n}{n^{2}}} \leq 2^{\frac{1}{4}} & \text { if } k=1, \\ \frac{n+1}{2} \mathrm{e}^{-\frac{n^{2}+2 n-3}{n^{2}} \log n} \leq \frac{n+1}{2 n} \leq 1 & \text { if } k \geq 2,\end{cases}
$$

by using the decreasing behavior with respect to $k$. Notice that $\rho_{1, n-1}$ is indeed much smaller than before (linear in $n$ whereas before it grew exponentially in $n$ ), but $B_{n}(\lambda)$ for $k=1$ is almost constant instead of linear in $n$.

In the general case,

$$
\rho_{k, l}=\prod_{j=l+1}^{n} \frac{k^{\prime}-\lambda_{j}+j-1}{k^{\prime}-\lambda_{j}+j-l-1} \leq \prod_{j=l+1}^{n} \frac{k+j-1}{k+j-l-1}
$$

with the usual notation $k^{\prime}=k+\lambda_{l+1}$. On the other hand, the transformation on partitions makes $B_{n}(\lambda)$ change by

$$
\frac{-l(n-l)\left(n+2 k^{\prime}-1\right)+2 l|\lambda|_{l+1, n}}{n^{2}}
$$

where $|\lambda|_{l+1, n}$ is the restricted size $\sum_{j=l+1}^{n} \lambda_{j}$. Notice now that

$$
-(n-l) k^{\prime}+|\lambda|_{l+1, n}=\sum_{j=l+1}^{n} \lambda_{j}-\lambda_{l+1}-k \leq \sum_{j=l+1}^{n}-k=-(n-l) k .
$$

So,

$$
\eta_{k, l} \leq \prod_{j=l+1}^{n} \frac{k+j-1}{k+j-l-1} \mathrm{e}^{-\frac{l(n-l)(n+2 k-1)}{n^{2}} \log n} \leq\left(\begin{array}{l}
n \\
l
\end{array}\right) \mathrm{e}^{-\frac{l(n-l)(n+1)}{n^{2}} \log n}
$$

which can as usual be estimated by Stirling (this is the same kind of computations as before). Hence, with $l \geq 3$, the last bound is always smaller than 1 , and also if $l=2$ unless $n=4$. If $n=4$ and $l=2$, then

$$
\eta_{k, 2} \leq \frac{(k+2)(k+3)}{k(k+1)} \mathrm{e}^{-\frac{3+2 k}{2} \log 2} \leq \begin{cases}\frac{3}{2^{3 / 2}} & \text { if } k=1 \\ 1 & \text { if } k \geq 2\end{cases}
$$


Finally, when $l=1$, one has exactly the same bound as for $l=n-1$, so $2^{\frac{1}{4}}$ when $k=1$ and 1 for $k=2$, Multiplying together all the bounds $\left(3 / 2^{\frac{3}{2}}\right.$ and twice $\left.2^{\frac{1}{4}}\right)$, we obtain:

Proposition 19 In the case of special unitary groups, at cut-off time,

$$
D^{\lambda} \mathrm{e}^{-\frac{t_{n, 0}}{2} B_{n}(\lambda)} \leq \frac{3}{2}
$$

for any integer partition $\lambda$ of length $n-1$.

Another big difference with the previous cases is that one cannot use Lemma 14 anymore. Indeed, for $\lambda=(k, \ldots, k)_{n-1}, B_{n}(\lambda)=\frac{k(n-1)}{n}=\frac{|\lambda|}{n}$, so there is no hope to have an inequality of the type $B_{n}(\lambda) \geq \alpha|\lambda|$ for any partition. That said, set $\delta_{i}=$ $\lambda_{i}-\lambda_{i+1} ;$ then,

$$
B_{n}(\lambda)=\frac{1}{n^{2}} \sum_{1 \leq i<j \leq n}\left(\lambda_{i}-\lambda_{j}\right)^{2}+\frac{1}{n} \sum_{i=1}^{n-1} i(n-i) \delta_{i} \geq \sum_{i=1}^{n-1} \frac{i(n-i)}{n} \delta_{i} .
$$

This leads us to study the series

$$
T_{n}(x)=\sum_{\delta_{1}, \ldots, \delta_{n-1} \geq 0} x^{\sum_{i=1}^{n-1} \frac{i(n-i)}{n} \delta_{i}}=\prod_{i=1}^{n-1} \frac{1}{1-x^{\frac{i(n-i)}{n}}} .
$$

Clearly, each $T_{n}(x)$ is convex on $\mathbb{R}_{+}$, so if we can show for example that $T_{n}\left(\frac{1}{8}\right)$ stays smaller than $1+\frac{K}{8}$ for every $n$, then we will also have the inequality $T_{n}(x) \leq 1+K x$ for every $0 \leq x \leq \frac{1}{8}$. Set $U_{n}(x)=\log \left(T_{n}(x)\right)$; one has

$$
U_{n}(x)=\sum_{i=1}^{n-1}-\log \left(1-x^{\frac{i(n-i)}{n}}\right) \leq \sum_{i=1}^{n-1} x^{\frac{i(n-i)}{n}} \leq 2 \sum_{i=1}^{\left\lfloor\frac{n}{2}\right\rfloor} x^{\frac{i}{2}} \leq \frac{2}{1-x^{\frac{1}{2}}}
$$

for $0 \leq x \leq \frac{1}{8}$. It follows that $T_{n}(x) \leq 1+K x$ with $K \leq 169$. Suppose $\frac{1}{n^{2 \varepsilon}} \leq \frac{1}{8}$. Then,

$$
\begin{aligned}
S_{n}\left(t_{n, \varepsilon}\right) & =\sum_{\lambda \in \mathfrak{Y}_{n-1}^{\star}}\left(D^{\lambda}\right)^{2} \mathrm{e}^{-t_{n, \varepsilon} B_{n}(\lambda)} \leq \frac{9}{4} \sum_{\lambda \in \mathfrak{Y}_{n-1}^{\star}}\left(\frac{1}{n^{2 \varepsilon}}\right)^{B_{n}(\lambda)} \\
& \leq \frac{9}{4}\left(T_{n}\left(\frac{1}{n^{2 \varepsilon}}\right)-1\right) \leq \frac{1521}{4 n^{2 \varepsilon}} \leq \frac{400}{n^{2 \varepsilon}}
\end{aligned}
$$

which leads to

$$
d_{\mathrm{TV}}^{\mathrm{SU}(n, \mathbb{C})}\left(\mu_{2(1+\varepsilon) \log n}, \text { Haar }\right) \leq \frac{10}{n^{\varepsilon}}
$$

If $\frac{1}{n^{2 \varepsilon}} \geq \frac{1}{8}$, then this inequality is also trivially satisfied. Hence, the case of special unitary groups is done. For the quotients $\mathrm{SU}(n) / \mathrm{SO}(n)$, one obtains

$$
S_{n}\left(\frac{t_{n, \varepsilon}}{2}\right) \leq \frac{3}{2}\left(T_{n}\left(\frac{1}{n^{\varepsilon}}\right)-1\right) \leq \frac{507}{2 n^{\varepsilon}} \leq \frac{256}{n^{\varepsilon}}
$$


and therefore

$$
d_{\mathrm{TV}}^{\mathrm{SU}(n, \mathbb{C}) / \mathrm{SO}(n, \mathbb{R})}\left(\mu_{(1+\varepsilon) \log n}, \text { Haar }\right) \leq \frac{8}{n^{\frac{\varepsilon}{2}}}
$$

The proof is exactly the same for $\operatorname{SU}(2 n) / \mathrm{USp}(n)$ and gives the same inequality, however with $(2 n)^{\frac{\varepsilon}{2}}$ instead of $n^{\frac{\varepsilon}{2}}$.

For the complex Grassmannian varieties, we have seen that it was easier to see them as quotients of $\mathrm{U}(n)$ (instead of $\mathrm{SU}(n)$ ), and this forces us to do some additional computations. Though the cut-off phenomenon also holds in the case of $\mathrm{U}(n)$, the set of irreducible representations is then labelled by sequences of possibly negative integers, which makes our scheme of growth of partitions a little bit more cumbersome to apply. Fortunately, for Grassmannians, the spherical representations can be labelled by true partitions, but then the dimensions are given by a different formula and we have to do once again the estimates of quotients $\rho_{k, l}$ and $\eta_{k, l}$. We refer to Appendix 5.3 for a proof of the following:

$$
A_{n}(\lambda) \mathrm{e}^{-\log n B_{n}(\lambda)} \leq 1
$$

for any partition. Then, one can compare directly $B_{n}(\lambda)$ to $|\lambda|$ :

$$
B_{n}(\lambda)=\frac{2}{n} \sum_{i=1}^{p} \lambda_{i}^{2}+(n+1-2 i) \lambda_{i} \geq 2 \sum_{i=1}^{p} \frac{i(n-i)}{n}\left(\lambda_{i}-\lambda_{i+1}\right) \geq \sum_{i=1}^{p} i\left(\lambda_{i}-\lambda_{i+1}\right)=|\lambda| .
$$

We conclude that

$$
S_{n}\left(\frac{t_{n, \varepsilon}}{2}\right) \leq \sum_{\lambda \in \mathfrak{Y}_{q}^{*}} \mathrm{e}^{-\varepsilon|\lambda| \log n} \leq \frac{5}{n^{\varepsilon}} \leq \frac{16}{n^{\varepsilon}} \quad ; \quad d_{\mathrm{TV}}^{\mathrm{Gr}(n, q, \mathbb{C})}\left(\mu_{(1+\varepsilon) \log n}, \text { Haar }\right) \leq \frac{2}{n^{\frac{\varepsilon}{2}}}
$$

and this ends the proof of all the upper bounds of type (Eq. 1.7).

\section{Lower Bounds Before the Cut-Off Time}

The proofs of the lower bounds before cut-off time rely on the following simple ideas, which appeared already in some cases for instance in [25, Section 7]. Denote $\lambda_{\min }$ the (spherical) irreducible representation "of minimal eigenvalue" identified in Section 3.1. We then consider the random variable:

$$
\Omega= \begin{cases}\chi^{\lambda_{\min }}(k) & \text { in the case of groups, } \\ \sqrt{D^{\lambda_{\min }}} \phi^{\lambda_{\min }}(g K) & \text { in the case of symmetric spaces of type non-group. }\end{cases}
$$

In this equation, $k$ or $g K$ will be taken at random either under the Haar measure of the space, or under a marginal law $\mu_{t}$ of the Brownian motion; we shall denote $\mathbb{E}_{\infty}$ and $\mathbb{E}_{t}$ the corresponding expectations. When $\Omega$ is real valued, we also denote $\operatorname{Var}_{\infty}$ and $\operatorname{Var}_{t}$ the corresponding variances:

$$
\operatorname{Var}[\Omega]=\mathbb{E}\left[\Omega^{2}\right]-\mathbb{E}[\Omega]^{2}=\mathbb{E}\left[(\Omega-\mathbb{E}[\Omega])^{2}\right] .
$$


In the case of unitary groups and their quotients, $\Omega$ will be complex valued, and we shall use the notations $\operatorname{Var}_{\infty}$ and $\operatorname{Var}_{t}$ for the expectation of the square of the module of $\Omega-\mathbb{E}[\Omega]$ :

$$
\operatorname{Var}[\Omega]=\mathbb{E}\left[|\Omega|^{2}\right]-|\mathbb{E}[\Omega]|^{2}=\mathbb{E}\left[|\Omega-\mathbb{E}[\Omega]|^{2}\right] .
$$

The normalization of Eq. 4.1 is actually chosen so that $\Omega$ is in any case of mean 0 and variance 1 under the Haar measure.

Remark In fact, much more is known about the asymptotic distribution of these functions under Haar measure, when $n$ goes to infinity; see [8]. For instance, over the unitary groups, the moments of order smaller than $n_{0}$ of $\chi^{(1,0, \ldots, 0)}(g)=\operatorname{tr} g$ agree with those of a standard complex gaussian variable as soon as $n$ is bigger than $n_{0}$. In particular, if $g$ is distributed according to the Haar measure of $\mathrm{U}(n, \mathbb{C})$, then tr $g$ converges (without any normalization) towards a standard complex gaussian variable. One has similar results for orthogonal and symplectic groups, this time with standard real gaussian variables. As far as we know, the same problem with spherical functions on the classical symmetric spaces is still open, and certain computations performed in this section are related to this question.

One will also prove that under a marginal law $\mu_{t}$, the variance of $\Omega$ stays small for every value of $t$, whereas its mean before cut-off time is big (not at all near zero). Standard methods of moments allow then to prove that the probability of a event

$$
E_{\alpha}=\{k|| \Omega(k) \mid \geq \alpha\} \quad \text { or } \quad\{g K|| \Omega(g K) \mid \geq \alpha\}
$$

is before cut-off time near 1 under $\mu_{t}$, and near 0 under Haar measure (for an adequate choice of $\alpha$ ). This is sufficient to prove the lower bounds, see Section 4.2; in other words, $\Omega$ is a discriminating random variable for the cut-off phenomenon.

The method presented above reduces the problem mainly to the expansion in irreducible characters or in spherical zonal functions of $\Omega^{2}$ or of $|\Omega|^{2}$; cf. Section 4.1. In the case of compact groups, this amounts simply to understand the tensor product of $V^{\lambda_{\min }}$ with itself, or with its conjugate when the character $\Omega$ is complex valued. However, for compact symmetric spaces of type non-group, this is far less obvious. Notice that a zonal spherical function $\phi^{\lambda}$ can be uniquely characterized by the following properties:

- it is a linear combination of matrix coefficients of the representation $V^{\lambda}$ :

$$
\phi^{\lambda}(g K)=\sum_{i=1}^{D^{\lambda}} \sum_{j=1}^{D^{\lambda}} c^{i j} \rho_{i j}^{\lambda}(g K) .
$$

- it is in $\mathscr{L}^{2}(G / K)^{K}$, i.e., it is $K$-bi-invariant; and it is normalized so that $\phi^{\lambda}(e K)=1$.

Consequently, if $\left(V^{\lambda_{\text {min }}}\right)^{\otimes 2}=V^{\nu_{1}} \oplus \cdots \oplus V^{\nu_{s}} \oplus V^{\epsilon_{1}} \oplus \cdots \oplus V^{\epsilon_{t}}$ with the $V^{\nu_{i}}$ spherical irreducible representations and the $V^{\epsilon_{j}}$ non-spherical irreducible representations, then there exists an expansion

$$
\left(\phi^{\lambda_{\min }}\right)^{2}=c_{v_{1}} \phi^{v_{1}}+c_{v_{2}} \phi^{v_{2}}+\cdots+c_{v_{s}} \phi^{v_{s}} .
$$


Nonetheless, it seems difficult to guess at the same time the values of the coefficients $c_{v}$ in this expansion. The only "easy" computation is the coefficient of the constant function in $\left(\phi^{\lambda}\right)^{2}$, or more generally in a product $\phi^{\lambda} \phi^{\rho}$ :

$$
c_{\phi^{1_{G}}}\left[\phi^{\lambda} \phi^{\rho}\right]=\int_{X} \phi^{\lambda}(x) \phi^{\rho}(x) d x= \begin{cases}0 & \text { if } \phi^{\rho} \neq \overline{\phi^{\lambda}} \\ \frac{1}{D^{\lambda}} & \text { otherwise }\end{cases}
$$

As far as we know, for a general zonal spherical function, there is a definitive solution to Eq. 4.2 only:

- $\quad$ in the case of symmetric spaces of rank 1, see [12];

- for the spherical functions of low degree of the pairs $(\mathrm{SU}(n), \mathrm{SO}(n))$ and $(\mathrm{SU}(2 n), \mathrm{USp}(n))$, because they are known to be Jack polynomials of parameter 2 or $1 / 2$; see [21, Chapter VII].

For our problem, one can fortunately give in every case a geometric description of the discriminating spherical representation and of the corresponding spherical vector. This yields an expression of $\phi^{\lambda_{\min }}(g K)$ as a degree 2 polynomial of the matrix coefficients of $g$. Now it turns out that the joint moments of these coefficients under $\mu_{t}$ and $\mu_{\infty}=$ Haar can be calculated by mean of the stochastic differential equations defining the $G$-valued Brownian motion; see Lemma 23, which we reproduce from [18, Proposition 1.4]. As $\left(\phi^{\lambda_{\min }}(g K)\right)^{2}$ or $\left|\phi^{\lambda_{\min }}(g K)\right|^{2}$ is also a polynomial in the coefficients $g_{i j}$, one can therefore compute its expectation under $\mu_{t}$, and this actually gives back the coefficients in the expansion (4.2). Thus, the algebraic difficulties raised in our proof of the lower bounds will be solved by arguments of stochastic analysis.

\subsection{Expansion of the Square of the Discriminating Zonal Spherical Functions}

The orthogonality of characters or of zonal spherical functions ensures that for every non-trivial (spherical) irreducible representation $\lambda$,

$$
\begin{aligned}
\mathbb{E}_{\infty}\left[\chi^{\lambda}\right] & =\mathbb{E}_{\infty}\left[\chi^{\lambda}(k) \chi^{\mathbf{1}_{K}}(k)\right]=\left\langle\chi^{\lambda} \mid \chi^{\mathbf{1}_{K}}\right\rangle_{\mathscr{L}^{2}(K)}=0 ; \\
\mathbb{E}_{\infty}\left[\sqrt{D^{\lambda}} \phi^{\lambda}\right] & =\sqrt{D^{\lambda}} \mathbb{E}_{\infty}\left[\phi^{\lambda}(g K) \phi^{\mathbf{1}_{G}}(g K)\right]=\sqrt{D^{\lambda}}\left\langle\phi^{\lambda} \mid \phi^{\mathbf{1}_{K}}\right\rangle_{\mathscr{L}^{2}(G / K)}=0 .
\end{aligned}
$$

The function corresponding to the trivial representation, which is just the constant function equal to 1 , has of course mean 1 under the Haar measure, and also under $\mu_{t}$. On the other hand, Theorem 11 allows one to compute the mean of a non-trivial irreducible character of zonal spherical function under $\mu_{t}$ :

$$
\begin{array}{r}
\mathbb{E}_{t}\left[\chi^{\lambda}\right]=\int_{K} p_{t}^{K}(k) \chi^{\lambda}(k) d k=\left[\chi^{\lambda}\right]\left(p_{t}^{K}\right)=D^{\lambda} \mathrm{e}^{-\frac{t}{2} B_{n}(\lambda)}=\left\{A_{n}(\lambda) \mathrm{e}^{-t B_{n}(\lambda)}\right\}^{\frac{1}{2}} \\
\mathbb{E}_{t}\left[\sqrt{D^{\lambda}} \phi^{\lambda}\right]=\sqrt{D^{\lambda}} \int_{X=G / K} p_{t}^{X}(x) \phi^{\lambda}(x) d x=\sqrt{D^{\lambda}} \frac{\left[\phi^{\lambda}\right]\left(p_{t}^{X}\right)}{D^{\lambda}}=\left\{A_{n}(\lambda) \mathrm{e}^{-t B_{n}(\lambda)}\right\}^{\frac{1}{2}}
\end{array}
$$

with the notations of Proposition 12, and where $\left[\chi^{\lambda}\right](f)$ or $\left[\phi^{\lambda}\right](f)$ denotes the coefficient of $\chi^{\lambda}$ or $\phi^{\lambda}$ in the expansion of $f$. So, with the help of the table of Lemma 13 , we can compute readily $\mathbb{E}_{t}[\Omega]$ in each case, and also $\mathbb{E}_{\infty}[\Omega]$. 
In order to estimate $\operatorname{Var}_{t}[\Omega]$ and $\operatorname{Var}_{\infty}[\Omega]$, we now need to find a representationtheoretic interpretation of either $\Omega^{2}$ when $\Omega$ is real-valued, or of $|\Omega|^{2}$ when $\Omega$ is complex-valued. We begin with compact groups:

Lemma 20 Suppose $G=\mathrm{SO}(2 n, \mathbb{R})$ or $\operatorname{SO}(2 n+1, \mathbb{R})$ or $\operatorname{USp}(n, \mathbb{H})$. Then $\Omega=$ $\chi^{(1,0, \ldots, 0)_{n}}$ is real-valued, and

$$
\Omega^{2}=\left(\chi^{(1,0, \ldots, 0)_{n}}\right)^{2}=\chi^{(2,0, \ldots, 0)_{n}}+\chi^{(1,1,0, \ldots, 0)_{n}}+\chi^{(0,0, \ldots, 0)_{n}} .
$$

On the other hand, when $G=\mathrm{SU}(n, \mathbb{C}), \Omega$ is complex-valued, and

$$
|\Omega|^{2}=\chi^{(1,0, \ldots, 0)_{n-1}} \chi^{(1, \ldots, 1)_{n-1}}=\chi^{(2,1, \ldots, 1)_{n-1}}+\chi^{(0,0, \ldots, 0)_{n-1}} .
$$

Proof In each case, $\Omega(k)=\operatorname{tr} k$, up to the map (Eq. 1.2) in the symplectic case; this explains why $\Omega$ is real-valued in the orthogonal and symplectic case, and complexvalued in the unitary case. Then, the simplest way to prove the identities 4.3 and 4.4 is by manipulating the Schur functions of type A, B, C and D; indeed, these polynomials evaluated on the eigenvalues are known to be the irreducible characters of the corresponding groups, see Section 2.3. We start with the special orthogonal groups. In type $\mathrm{B}_{n},\left(z_{1}+\cdots+z_{n}+z_{1}^{-1}+\cdots+z_{n}^{-1}+1\right)^{2}$ is indeed equal to the sum of the three terms

$$
\begin{aligned}
s b_{(2,0, \ldots, 0)}\left(Z, Z^{-1}, 1\right)= & \left(\sum_{1 \leq i \leq j \leq n} z_{i} z_{j}+z_{i} z_{j}^{-1}+z_{i}^{-1} z_{j}+z_{i}^{-1} z_{j}^{-1}\right) \\
& +\left(\sum_{i=1}^{n} z_{i}+z_{i}^{-1}\right)-n ; \\
s b_{(1,1,0, \ldots, 0)}\left(Z, Z^{-1}, 1\right)= & \left(\sum_{1 \leq i<j \leq n} z_{i} z_{j}+z_{i} z_{j}^{-1}+z_{i}^{-1} z_{j}+z_{i}^{-1} z_{j}^{-1}\right) \\
& +\left(\sum_{i=1}^{n} z_{i}+z_{i}^{-1}\right)+n ; \\
s b_{(0, \ldots, 0)}\left(Z, Z^{-1}, 1\right)= & 1 ;
\end{aligned}
$$

whereas in type $\mathrm{D}_{n},\left(z_{1}+\cdots+z_{n}+z_{1}^{-1}+\cdots+z_{n}^{-1}\right)^{2}$ is equal to the sum of the three terms

$$
\begin{aligned}
s d_{(2,0, \ldots, 0)}\left(Z, Z^{-1}\right) & =\left(\sum_{1 \leq i \leq j \leq n} z_{i} z_{j}+z_{i} z_{j}^{-1}+z_{i}^{-1} z_{j}+z_{i}^{-1} z_{j}^{-1}\right)-n-1 ; \\
\operatorname{sd}_{(1,1,0, \ldots, 0)}\left(Z, Z^{-1}\right) & =\left(\sum_{1 \leq i<j \leq n} z_{i} z_{j}+z_{i} z_{j}^{-1}+z_{i}^{-1} z_{j}+z_{i}^{-1} z_{j}^{-1}\right)+n \\
\operatorname{sd}_{(0, \ldots, 0)}\left(Z, Z^{-1}\right) & =1
\end{aligned}
$$


For compact symplectic groups, hence in type $\mathrm{C}_{n},\left(z_{1}+\cdots+z_{n}+z_{1}^{-1}+\cdots+z_{n}^{-1}\right)^{2}$ is indeed equal to the sum of the three terms

$$
\begin{aligned}
\operatorname{sc}_{(2,0, \ldots, 0)}\left(Z, Z^{-1}\right) & =\left(\sum_{1 \leq i \leq j \leq n} z_{i} z_{j}+z_{i} z_{j}^{-1}+z_{i}^{-1} z_{j}+z_{i}^{-1} z_{j}^{-1}\right)-n ; \\
\operatorname{sc}_{(1,1,0, \ldots, 0)}\left(Z, Z^{-1}\right) & =\left(\sum_{1 \leq i<j \leq n} z_{i} z_{j}+z_{i} z_{j}^{-1}+z_{i}^{-1} z_{j}+z_{i}^{-1} z_{j}^{-1}\right)+n-1 ; \\
\operatorname{sc}_{(0, \ldots, 0)}\left(Z, Z^{-1}\right) & =1 ;
\end{aligned}
$$

and this is also $\left(s c_{(1,0, \ldots, 0)}\left(Z, Z^{-1}\right)\right)^{2}=\left(\chi^{(1,0, \ldots, 0)}(k)\right)^{2}=\Omega(k)^{2}$. Thus, formula 4.3 is proved. In type $\mathrm{A}_{n-1}$, notice that for every character $\chi^{\lambda}, \overline{\chi^{\lambda}(k)}=\chi^{\lambda}\left(k^{-1}\right)=\chi^{\lambda^{*}}(k)$, where $\lambda^{*}$ is the sequence obtained from $\lambda$ by the simple transformation

$$
\left(\lambda_{1} \geq \lambda_{2} \geq \cdots \geq \lambda_{n-1}\right)_{n-1} \mapsto\left(\lambda_{1} \geq \lambda_{1}-\lambda_{n-1} \geq \cdots \geq \lambda_{1}-\lambda_{2}\right)_{n-1} .
$$

Indeed, if $z_{1}, \ldots, z_{n}$ are the eigenvalues of $k$, then

$$
\begin{aligned}
\overline{\chi^{\lambda}(k)} & =s_{\left(\lambda_{1}, \ldots, \lambda_{n-1}\right)_{n-1}}\left(z_{1}^{-1}, \ldots, z_{n}^{-1}\right)=s_{\left(\lambda_{1}, \ldots, \lambda_{n-1}, 0\right)_{n}}\left(z_{1}^{-1}, \ldots, z_{n}^{-1}\right) \\
& =s_{\left(0,-\lambda_{n-1}, \ldots,-\lambda_{1}\right)_{n}}\left(z_{n}, \ldots, z_{1}\right)=s_{\left(\lambda_{1}, \lambda_{1}-\lambda_{n-1}, \ldots, 0\right)_{n}}\left(z_{1}, \ldots, z_{n}\right) \\
& =s_{\left(\lambda_{1}, \lambda_{1}-\lambda_{n-1}, \ldots, \lambda_{1}-\lambda_{2}\right)_{n-1}}\left(z_{1}, \ldots, z_{n}\right)=\chi^{\lambda^{*}}(k)
\end{aligned}
$$

Here, one uses the relation $z_{1} z_{2} \cdots z_{n}=1$ for every element of the torus of $\operatorname{SU}(n, \mathbb{C})$, which enables one to transform a $n$-vector of possibly negative integers into a $(n-1)$-vector of non-negative integers. In particular, $|\Omega(k)|^{2}=\left|\chi^{(1,0, \ldots, 0)_{n-1}}(k)\right|^{2}=$ $\chi^{(1,0, \ldots, 0)_{n-1}}(k) \chi^{(1,1, \ldots, 1)_{n-1}}(k)$. Then, a simple calculation with symmetric functions yields formula 4.4 :

$$
\begin{aligned}
& \chi^{(1,0, \ldots, 0)_{n-1}}(k) \chi^{(1,1, \ldots, 1)_{n-1}}(k)=\left(z_{1}+\cdots+z_{n}\right)\left(z_{1}^{-1}+\cdots+z_{n}^{-1}\right) \\
& =\left(n-1+\sum_{i<j} z_{i} z_{j}^{-1}+z_{i}^{-1} z_{j}\right)+1 \\
& =s_{(1,0, \ldots, 0,-1)_{n}}(Z)+s_{(0, \ldots, 0)_{n}}(Z) \\
& =s_{(2,1, \ldots, 1)_{n-1}}(Z)+s_{(0, \ldots, 0)_{n-1}}(Z) \\
& =\chi^{(2,1, \ldots, 1)_{n-1}}(k)+\chi^{(0, \ldots, 0)_{n-1}(k)}
\end{aligned}
$$

where $Z=\left\{z_{1}, \ldots, z_{n}\right\}$ is the alphabet of the eigenvalues of $k$.

\subsubsection{Values of the Zonal Functions and Abstract Expansions of their Squares}

As explained in the introduction of this part, the case of compact symmetric spaces of type non-group is much more involved. We start by finding an expression of $\Omega(g K)$ in terms of the matrix coefficients $g_{i j}$ of the matrix $g$. 
Proposition 21 In terms of the matrix coefficients of $g, \phi^{\lambda_{\min }}(g K)$ is given by:

\begin{tabular}{cccc}
\hline$G / K$ & $V^{\lambda} \min$ & $\phi^{\lambda} \min (g K)$ & $\mathbb{k}$ \\
\hline $\operatorname{Gr}(n, q, \mathbb{R})$ & $\mathfrak{s o}^{\perp}(n, \mathbb{C})$ & $\frac{1}{p} \sum_{i=1}^{p} \sum_{j=1}^{p}\left(g_{i j}\right)^{2}+\frac{1}{q} \sum_{i=p+1}^{n} \sum_{j=p+1}^{n}\left(g_{i j}\right)^{2}-1$ & $\mathbb{R}$ \\
$\operatorname{Gr}(n, q, \mathbb{C})$ & $\mathfrak{s l}(n, \mathbb{C})$ & $\frac{1}{p} \sum_{i=1}^{p} \sum_{j=1}^{p}\left|g_{i j}\right|^{2}+\frac{1}{q} \sum_{i=p+1}^{n} \sum_{j=p+1}^{n}\left|g_{i j}\right|^{2}-1$ & $\mathbb{R}$ \\
$\operatorname{Gr}(n, q, \mathbb{H})$ & $\mathfrak{s p}^{\perp}(2 n, \mathbb{C})$ & $\frac{1}{p} \sum_{i=1}^{p} \sum_{j=1}^{p}\left|g_{i j}\right|^{2}+\frac{1}{q} \sum_{i=p+1}^{n} \sum_{j=p+1}^{n}\left|g_{i j}\right|^{2}-1$ & $\mathbb{R}$ \\
$\mathrm{SO}(2 n, \mathbb{R}) / \mathrm{U}(n, \mathbb{C})$ & $\mathcal{A}^{2}\left(\mathbb{C}^{2 n}\right)$ & $\frac{1}{n} \sum_{i=1}^{n} \sum_{j=1}^{n} g_{(2 i)(2 j)} g_{(2 i-1)(2 j-1)}-g_{(2 i)(2 j-1)} g_{(2 i-1)(2 j)}$ & $\mathbb{R}$ \\
$\mathrm{SU}(n, \mathbb{C}) / \mathrm{SO}(n, \mathbb{R})$ & $\mathcal{S}^{2}\left(\mathbb{C}^{n}\right)$ & $\frac{1}{n} \sum_{i=1}^{n} \sum_{j=1}^{n}\left(g_{i j}\right)^{2}$ & $\mathbb{C}$ \\
$\mathrm{SU}(2 n, \mathbb{C}) / \mathrm{USp}(n, \mathbb{H})$ & $\mathcal{A}^{2}\left(\mathbb{C}^{2 n}\right)$ & $\frac{1}{n} \sum_{i=1}^{n} \sum_{j=1}^{n} g_{(2 i)(2 j)} g_{(2 i-1)(2 j-1)}-g_{(2 i)(2 j-1)} g_{(2 i-1)(2 j)}$ & $\mathbb{C}$ \\
$\mathrm{USp}(n, \mathbb{H}) / \mathrm{U}(n, \mathbb{C})$ & $\mathcal{S}^{2}\left(\mathbb{C}^{2 n}\right)$ & $\frac{1}{n} \sum_{i=1}^{n} \sum_{j=1}^{n}\left([1]\left(g_{i j}\right)\right)^{2}+\left([\mathrm{j}]\left(g_{i j}\right)\right)^{2}-\left([\mathrm{i}]\left(g_{i j}\right)\right)^{2}-\left([\mathrm{k}]\left(g_{i j}\right)\right)^{2}$ & $\mathbb{R}$ \\
\hline
\end{tabular}

For real Grassmannians, $\mathfrak{s o}^{\perp}(n, \mathbb{C})$ denotes the orthogonal complement of $\mathfrak{s o}(n, \mathbb{C})$ in $\mathfrak{s l}(n, \mathbb{C})$; and for quaternionic Grassmannians, $\mathfrak{s p}^{\perp}(2 n, \mathbb{C})$ denotes the orthogonal complement of $\mathfrak{s p}(2 n, \mathbb{C})$ in $\mathfrak{s l}(2 n, \mathbb{C})$.

Proof Each space $V^{\lambda_{\min }}$ described in the statement of our proposition is endowed with a natural action of $G=\mathrm{SO}(n)$ or $\mathrm{SU}(n)$ or $\operatorname{USp}(n)$, namely, the action by conjugation in the case of Grassmannian varieties, and the diagonal action on tensors in the case of spaces of structures. Then, to say that

$$
\begin{aligned}
& V_{\mathrm{SO}(n, \mathbb{R})}^{\left.(2,0, \ldots, 0)^{n}\right\rfloor}=\mathfrak{s o}^{\perp}(n, \mathbb{C}) ; \quad V_{\mathrm{U}(n, \mathbb{C})}^{(1,0, \ldots, 0,-1)_{n}}=\mathfrak{s l}(n, \mathbb{C}) ; V_{\mathrm{USp}(n, \mathbb{H})}^{(1,1,0, \ldots, 0)_{n}}=\mathfrak{s p}^{\perp}(2 n, \mathbb{C}) ; \\
& V_{\mathrm{SO}(2 n, \mathbb{R})}^{(1,1,0, \ldots, 0)_{n}}=\mathcal{A}^{2}\left(\mathbb{C}^{n}\right) ; \quad V_{\mathrm{SU}(n, \mathbb{C})}^{(2,0, \ldots)_{n-1}}=\mathcal{S}^{2}\left(\mathbb{C}^{n}\right) ; V_{\mathrm{SU}(2 n, \mathbb{C})}^{(1,1,0, \ldots, 0)_{2 n-1}}=\mathcal{A}^{2}\left(\mathbb{C}^{2 n}\right) ; \\
& V_{\mathrm{USp}(n, \mathbb{H})}^{\left.(2,0, \ldots, 0)_{n}\right)}=\mathcal{S}^{2}\left(\mathbb{C}^{2 n}\right)
\end{aligned}
$$

is equivalent to the following statements: the trace of $g \in \mathrm{SO}(n, \mathbb{R})$ acting on $\mathfrak{s o}^{\perp}(n, \mathbb{C})$ is given by the Schur function of type B or D and label $(2,0, \ldots, 0)_{\left\lfloor\frac{n}{2}\right\rfloor}$; the trace of $g \in \mathrm{U}(n, \mathbb{C})$ acting on $\mathfrak{s l}(n, \mathbb{C})$ is given by the Schur function of type A and label $(1,0, \ldots, 0,-1)_{n} ;$ etc. Let us detail for instance this last case. We have seen in the previous Lemma that

$$
s_{(1,0, \ldots, 0,-1)_{n}}(Z)=\left(z_{1}+\cdots+z_{n}\right)\left(z_{1}^{-1}+\cdots+z_{n}^{-1}\right)-1
$$

On the other hand, the module $\mathfrak{g l}(n, \mathbb{C})$ on which $\mathrm{SU}(n, \mathbb{C})$ acts by conjugation is the tensor product of modules $\left(\mathbb{C}^{n}\right) \otimes\left(\mathbb{C}^{n}\right)^{*}$. It follows that the trace of the action by conjugation of $g \in \mathrm{SU}(n, \mathbb{C})$ on $\mathfrak{g l}(n, \mathbb{C})$ is

$$
\chi(g)=(\operatorname{trg})\left(\operatorname{tr}\left(g^{-1}\right)^{t}\right)=\left(z_{1}+\cdots+z_{n}\right)\left(z_{1}^{-1}+\cdots+z_{n}^{-1}\right)
$$

if $z_{1}, \ldots, z_{n}$ are the eigenvalues of $g$. Subtracting 1 amounts to look at the irreducible submodule $\mathfrak{s l}(n, \mathbb{C})$ inside $\mathfrak{g l}(n, \mathbb{C})$. The other cases are entirely similar, and the corresponding values of the Schur functions have all been computed in Lemma 20.

Once the discriminating representations have been given a geometric interpretation, it is easy to find the corresponding $K$-invariant (spherical) vectors. We endow each space of matrices with the invariant scalar product $\langle M \mid N\rangle=\operatorname{tr} M N^{\dagger}$, and each space of tensors with the scalar product $\left\langle x_{1} \otimes x_{2} \mid y_{1} \otimes y_{2}\right\rangle=\left\langle x_{1} \mid y_{1}\right\rangle\left\langle x_{2} \mid y_{2}\right\rangle$, where 
$\langle v \mid w\rangle$ is the usual Hermitian scalar product on $\mathbb{C}^{n}$ or $\mathbb{C}^{2 n}$. We also denote $\left(e_{i}\right)_{i}$ the canonical basis of $\mathbb{C}^{n}$ or $\mathbb{C}^{2 n}$. Then, the $K$-spherical vectors write as:

\begin{tabular}{ccc}
\hline$G$ & $K$ & $e^{\lambda_{\min }}$ \\
\hline $\mathrm{SO}(n)$ & $\mathrm{SO}(p) \times \mathrm{SO}(q)$ & $\frac{1}{\sqrt{n p q}}\left(\begin{array}{cc}-q I_{p} & 0 \\
0 & p I_{q}\end{array}\right)$ \\
$\mathrm{SU}(n)$ & $\mathrm{S}(U(p) \times U(q))$ & $\frac{1}{\sqrt{n p q}}\left(\begin{array}{cc}-q I_{p} & 0 \\
0 & p I_{q}\end{array}\right)$ \\
$\mathrm{USp}(n)$ & $\mathrm{USp}(p) \times \mathrm{USp}(q)$ & $\frac{1}{\sqrt{2 n p q}}\left(\begin{array}{cc}-q I_{2 p} & 0 \\
0 & p I_{2 q}\end{array}\right)$ \\
$\mathrm{SO}(2 n)$ & $\mathrm{U}(n)$ & $\frac{1}{\sqrt{2 n}} \sum_{i=1}^{n} e_{2 i} \otimes e_{2 i-1}-e_{2 i-1} \otimes e_{2 i}$ \\
$\mathrm{SU}(n)$ & $\mathrm{SO}(n)$ & $\frac{1}{\sqrt{n}} \sum_{i=1}^{n} e_{i} \otimes e_{i}$ \\
$\mathrm{SU}(2 n)$ & $\mathrm{USp}(n)$ & $\frac{1}{\sqrt{2 n}} \sum_{i=1}^{n} e_{2 i} \otimes e_{2 i-1}-e_{2 i-1} \otimes e_{2 i}$ \\
$\mathrm{USp}(n)$ & $\mathrm{U}(n)$ & $\frac{1}{\sqrt{2 n}} \sum_{i=1}^{2 n} e_{i} \otimes e_{i}$ \\
\hline
\end{tabular}

In each case, $e^{\lambda_{\min }}$ belongs trivially to $V^{\lambda_{\min }}$ and is of norm 1 , so the only thing to check then is the $K$-invariance. In the case of Grassmannian varieties, the matrix $e^{\lambda_{\min }}$ commutes indeed with $\mathrm{G}(p) \times \mathrm{G}(q)$, since it is also $(p, q)$-block-diagonal and with scalar multiples of the identity matrix in each diagonal block. The notation $\operatorname{USp}(n, \mathbb{H})$ used in this paper was meant to avoid any confusion between $\operatorname{Sp}(2 n, \mathbb{C})$ and its compact form, the compact symplectic group. For $\mathrm{U}(n)$ inside $\mathrm{SO}(2 n)$, we use the well-known fact that inside $\operatorname{SL}(2 n, \mathbb{C})$,

$$
\mathrm{SO}(2 n, \mathbb{R}) \cap \operatorname{Sp}(2 n, \mathbb{C}) \simeq \mathrm{U}(n, \mathbb{C}),
$$

the isomorphism being given by the map (Eq. 1.1). This implies in particular that $\mathrm{U}(n)$ leaves invariant the skew-symmetric tensor $\sum_{i=1}^{n} e_{2 i} \otimes e_{2 i-1}-e_{2 i-1} \otimes e_{2 i}$ corresponding to the skew-symmetric form defining $\operatorname{Sp}(2 n, \mathbb{C})$. The intersection formula 4.6 also proves that $\mathrm{U}(n)$ leaves invariant the symmetric tensor $\sum_{i=1}^{2 n} e_{i} \otimes e_{i}$, whence the value of the spherical vector for $\mathrm{U}(n)$ inside $\mathrm{USp}(n)$. Finally, for $\mathrm{SO}(n)$ inside $\mathrm{SU}(n)$ and $\mathrm{USp}(n)$ inside $\mathrm{SU}(2 n)$, we use again the defining symmetric bilinear form or skew-symmetric bilinear form associated to the group $K$ to construct a $K$ invariant vector.

The value of $\phi^{\lambda_{\min }}$ is then given by the formula $\phi^{\lambda}(g)=\left\langle e^{\lambda} \mid \rho^{\lambda}(g) e^{\lambda}\right\rangle$, that is to say

$$
\operatorname{tr}\left(M_{p, q} g M_{p, q} g^{t}\right) \quad ; \quad \operatorname{tr}\left(M_{p, q} g M_{p, q} g^{\dagger}\right) \quad ; \quad \frac{1}{2} \operatorname{tr}\left(\tilde{M}_{p, q} \widetilde{g} \tilde{M}_{p, q} \widetilde{g}^{\dagger}\right)
$$

for real, complex and quaternionic Grassmannians;

$$
\frac{1}{n} \sum_{i, j=1}^{n}\left(g_{i j}\right)^{2} ; \quad \frac{1}{2 n} \sum_{i, j=1}^{2 n}\left(\widetilde{g}_{i j}\right)^{2}
$$

for $\mathrm{SU}(n) / \mathrm{SO}(n)$ and $\mathrm{USp}(n) / \mathrm{U}(n)$; and

$$
\frac{1}{n} \sum_{i=1}^{n} \sum_{j=1}^{n} g_{(2 i)(2 j)} g_{(2 i-1)(2 j-1)}-g_{(2 i)(2 j-1)} g_{(2 i-1)(2 j)}
$$


for $\mathrm{SO}(2 n) / \mathrm{U}(n)$ and $\mathrm{SU}(2 n) / \mathrm{USp}(n)$. Here by $\tilde{g}$ we mean the complex matrix of size $2 n \times 2 n$ obtained from a quaternionic matrix of size $n \times n$ by the map (Eq. 1.2). In this last case, the computations can in fact be done inside $\mathrm{M}(n, \mathbb{H})$ : indeed,

$$
\begin{aligned}
& \left(\widetilde{g}_{(2 i-1)(2 j-1)}\right)^{2}+\left(\widetilde{g}_{(2 i-1)(2 j)}\right)^{2}+\left(\widetilde{g}_{(2 i)(2 j-1)}\right)^{2}+\left(\widetilde{g}_{(2 i)(2 j)}\right)^{2} \\
& \quad=2\left(\left([1]\left(g_{i j}\right)\right)^{2}+\left([\mathrm{j}]\left(g_{i j}\right)\right)^{2}-\left([\mathrm{i}]\left(g_{i j}\right)\right)^{2}-\left([\mathrm{k}]\left(g_{i j}\right)\right)^{2}\right),
\end{aligned}
$$

whereas $\widetilde{M}^{\star}=(\widetilde{M})^{\dagger}$ and $\frac{1}{2} \operatorname{tr} \widetilde{M}=\Re(\operatorname{tr} M)$. Thus, the formulas for the discriminating spherical functions of the spaces of structures are entirely proved, and for Grassmannian varieties, it suffices to check that for any unitary quaternionic matrix $N$,

$$
\Re\left(\operatorname{tr} M_{p, q} N M_{p, q} N^{\star}\right)=\frac{1}{p} \sum_{i=1}^{p} \sum_{j=1}^{p}\left|g_{i j}\right|^{2}+\frac{1}{q} \sum_{i=p+1}^{n} \sum_{j=p+1}^{n}\left|g_{i j}\right|^{2}-1 ;
$$

indeed the real and complex cases are specializations of this formula. This is easily done.

Lemma 22 There exists coefficients $a, b, c, \ldots$ (different on each line, and depending on $n$ and $q$ ) such that the following expansions hold:

$$
\begin{aligned}
& \operatorname{Gr}(n, q, \mathbb{R}):\left(\phi^{(2,0, \ldots, 0)\left\lfloor\frac{n}{2}\right\rfloor}\right)^{2}=\frac{2}{n^{2}+n-2}+a \phi^{(2,0, \ldots, 0)\left\lfloor\frac{n}{2}\right\rfloor}+b \phi^{(1,1,0 \ldots, 0)\left\lfloor\frac{n}{2}\right\rfloor} \\
& +c \phi^{(2,2,0, \ldots, 0)\left\lfloor\frac{n}{2}\right\rfloor}+d \phi^{(3,1,0, \ldots, 0)\left\lfloor\frac{n}{2}\right\rfloor}+e \phi^{(4,0, \ldots, 0)\left\lfloor\frac{n}{2}\right\rfloor} ; \\
& \operatorname{Gr}(n, q, \mathbb{C}):\left(\phi^{(2,1, \ldots, 1)_{n-1}}\right)^{2}=\frac{1}{n^{2}-1}+a \phi^{(2,1, \ldots, 1)_{n-1}}+b \phi^{(4,2, \ldots, 2)_{n-1}}+c \phi^{(2,2,1, \ldots, 1,0)_{n-1}} \text {; } \\
& \operatorname{Gr}(n, q, \mathbb{H}):\left(\phi^{(1,1,0, \ldots, 0)_{n}}\right)^{2}=\frac{1}{2 n^{2}-n-1}+a \phi^{\left(1^{2}, 0, \ldots, 0\right)_{n-1}}+b \phi^{\left(1^{4}, 0, \ldots, 0\right)_{n}}+c \phi^{(2,2,0, \ldots, 0)_{n}} ; \\
& \mathrm{SO}(2 n) / \mathrm{U}(n):\left(\phi^{(1,1,0, \ldots, 0)_{n}}\right)^{2}=\frac{1}{2 n^{2}-n}+a \phi^{\left(1^{2}, 0, \ldots, 0\right)_{n}}+b \phi^{\left(1^{4}, 0, \ldots, 0\right)_{n}}+c \phi^{(2,2,0, \ldots, 0)_{n}} \text {; } \\
& \mathrm{SU}(n) / \mathrm{SO}(n):\left|\phi^{(2,0, \ldots, 0)_{n-1}}\right|^{2}=\frac{2}{n^{2}+n}+a \phi^{(4,2, \ldots, 2)_{n-1}} \text {; } \\
& \operatorname{SU}(2 n) / \operatorname{USp}(n):\left|\phi^{(1,1,0, \ldots, 0)_{2 n-1}}\right|^{2}=\frac{1}{2 n^{2}-n}+a \phi^{(2,2,1, \ldots, 1,0)_{2 n-1}} \text {; } \\
& \mathrm{USp}(n) / \mathrm{U}(n):\left(\phi^{(2,0, \ldots, 0)_{n}}\right)^{2}=\frac{1}{2 n^{2}+n}+a \phi^{(2,0, \ldots, 0)_{n}}+b \phi^{(2,2,0, \ldots, 0)_{n}}+c \phi^{(4,0, \ldots, 0)_{n}} \text {. }
\end{aligned}
$$

In these formulas, it is understood that if the label $\lambda$ of the spherical function $\phi^{\lambda}$ does not make sense for a choice of $n$ and $q$, then this term does not appear in the expansion.

Proof Each time, one computes the expansion in irreducible representations of $V^{\lambda_{\min }} \otimes V^{\lambda_{\min }}$ in the case of real-valued spherical functions, and of $V^{\lambda_{\min }} \otimes V^{\lambda_{\min }^{*}}$ in the case of complex-valued spherical functions, where $\lambda \mapsto \lambda^{*}$ is the transformation of weights given by Eq. 4.5. This expansion can be found with Schur functions; let us 
detail for instance the case of complex Grassmannian varieties $\operatorname{Gr}(n, q, \mathbb{C})$. With an alphabet of eigenvalues $Z=\left\{z_{1}, \ldots, z_{n}\right\}$ such that $z_{1} z_{2} \cdots z_{n}=1$, one has

$$
\begin{aligned}
s_{(0, \ldots, 0)_{n-1}}(Z) & =1 \\
s_{(2,1, \ldots, 1)_{n-1}}(Z) & =s_{(1,0, \ldots, 0,-1)_{n}}(Z)=\left(\sum_{i, j=1}^{n} z_{i} z_{j}^{-1}\right)-1 \\
s_{(4,2, \ldots, 2)_{n-1}}(Z) & =s_{(2,0, \ldots, 0,-2)_{n}}(Z)=\left(\sum_{1 \leq i \leq j \leq n} \sum_{1 \leq k \leq l \leq n} z_{i} z_{j} z_{k}^{-1} z_{l}^{-1}\right)-\left(\sum_{i, j=1}^{n} z_{i} z_{j}^{-1}\right) \\
s_{(2,2,1, \ldots, 1,0)_{n-1}}(Z) & =s_{(1,1,0, \ldots, 0,-1,-1)_{n}}(Z)=\left(\sum_{1 \leq i<j \leq n} \sum_{1 \leq k<l \leq n} z_{i} z_{j} z_{k}^{-1} z_{l}^{-1}\right)-\left(\sum_{i, j=1}^{n} z_{i} z_{j}^{-1}\right) \\
s_{(3,1, \ldots, 1,0)_{n-1}}(Z) & =s_{(2,0, \ldots, 0,-1,-1)_{n}}(Z)=\left(\sum_{1 \leq i \leq j \leq n} \sum_{1 \leq k<l \leq n} z_{i} z_{j} z_{k}^{-1} z_{l}^{-1}\right)-\left(\sum_{i, j=1}^{n} z_{i} z_{j}^{-1}\right)+1 \\
s_{(3,3,2, \ldots, 2)_{n-1}}(Z) & =s_{(1,1,0, \ldots, 0,-2)_{n}}(Z)=\left(\sum_{1 \leq i<j \leq n} \sum_{1 \leq k \leq l \leq n} z_{i} z_{j} z_{k}^{-1} z_{l}^{-1}\right)-\left(\sum_{i, j=1}^{n} z_{i} z_{j}^{-1}\right)+1 .
\end{aligned}
$$

Consequently,

$$
\begin{aligned}
\left(V^{(2,1, \ldots, 1)_{n-1}}\right)^{\otimes 2}= & V^{(0, \ldots, 0)_{n-1}} \oplus 2 V^{(2,1, \ldots, 1)_{n-1}} \oplus V^{(4,2, \ldots, 2)_{n-1}} \oplus V^{(2,2,1, \ldots, 1,0)_{n-1}} \\
& \oplus V^{(3,3,2, \ldots, 2)_{n-1}} \oplus V^{(3,1, \ldots, 1,0)_{n-1}},
\end{aligned}
$$

because the same equality with Schur functions holds. The second line corresponds to non spherical representations, so only the terms of the first line can contribute to $\left(\phi^{(2,1, \ldots, 1)_{n-1}}\right)^{2}$. Entirely similar calculations yield:

- $\operatorname{Gr}(n, q, \mathbb{R})$ :

$$
\begin{aligned}
\left(V^{(2,0, \ldots, 0)\left\lfloor\left\lfloor\frac{n}{2}\right\rfloor\right.}\right)^{\otimes 2}= & V^{(0, \ldots, 0)\left\lfloor\frac{n}{2}\right\rfloor} \oplus V^{(2,0, \ldots, 0)\left\lfloor\frac{n}{2}\right\rfloor} \oplus V^{(1,1,0 \ldots, 0)\left\lfloor\frac{n}{2}\right\rfloor} \\
& \oplus V^{(2,2,0, \ldots, 0)\left\lfloor\frac{n}{2}\right\rfloor} \oplus V^{(3,1,0, \ldots, 0)\left\lfloor\frac{n}{2}\right\rfloor} \oplus V^{(4,0, \ldots, 0)\left\lfloor\frac{n}{2}\right\rfloor}
\end{aligned}
$$

- $\operatorname{Gr}(n, q, \mathbb{H})$ :

$$
\begin{aligned}
\left(V^{(1,1,0, \ldots, 0)_{n}}\right)^{\otimes 2}= & V^{(0, \ldots, 0)_{n}} \oplus V^{(1,1,0, \ldots, 0)_{n}} \oplus V^{(1,1,1,1,0, \ldots, 0)_{n}} \oplus V^{(2,2,0, \ldots, 0)_{n}} \\
& \oplus V^{(2,1,1,0, \ldots, 0)_{n}} .
\end{aligned}
$$

Only the terms on the first line are spherical.

- $\operatorname{SO}(2 n, \mathbb{R}) / \mathrm{U}(n, \mathbb{C})$ :

$$
\begin{aligned}
\left(V^{(1,1,0, \ldots, 0)_{n}}\right)^{\otimes 2}= & V^{(0, \ldots, 0)_{n}} \oplus V^{(1,1,0, \ldots, 0)_{n}} \oplus V^{(1,1,1,1,0, \ldots, 0)_{n}} \oplus V^{(2,2,0, \ldots, 0)_{n}} \\
& \oplus V^{(2,0, \ldots, 0)_{n}} \oplus V^{(2,1,1,0, \ldots, 0)_{n}},
\end{aligned}
$$

again with non-spherical representations gathered on the second line. 
- $\operatorname{SU}(n, \mathbb{C}) / \mathrm{SO}(n, \mathbb{R})$ :

$$
V^{(2,0, \ldots, 0)_{n-1}} \otimes V^{(2,2, \ldots, 2)_{n-1}}=V^{(0, \ldots, 0)_{n-1}} \oplus V^{(4,2, \ldots, 2)_{n-1}} \oplus V^{(2,1, \ldots, 1)_{n-1}},
$$

and the last term is not a spherical representation.

- $\operatorname{SU}(2 n, \mathbb{C}) / \mathrm{USp}(n, \mathbb{H})$ :

$$
V^{(1,1,0, \ldots, 0)_{2 n-1}} \otimes V^{(1, \ldots, 1,0)_{2 n-1}}=V^{(0, \ldots, 0)_{2 n-1}} \oplus V^{(2,2,1, \ldots, 1,0)_{2 n-1}} \oplus V^{(2,1, \ldots, 1)_{2 n-1}},
$$

and again the last term is not spherical.

- $\operatorname{USp}(n, \mathbb{H}) / \mathrm{U}(n, \mathbb{C})$ :

$$
\begin{aligned}
\left(V^{(2,0, \ldots, 0)_{n}}\right)^{\otimes 2}= & V^{(0, \ldots, 0)_{n}} \oplus V^{(2,0, \ldots, 0)_{n}} \oplus V^{(2,2, \ldots, 0)_{n}} \oplus V^{(4,0, \ldots, 0)_{n}} \\
& \oplus V^{(1,1,0, \ldots, 0)_{n}} \oplus V^{(3,1,0, \ldots, 0)_{n}} .
\end{aligned}
$$

The terms on the second line corresponds to non-spherical representations.

As mentioned before, the coefficient of the constant function in $\left|\phi^{\lambda_{\min }}\right|^{2}$ is then always equal to $\frac{1}{D^{\lambda_{\min }}}$.

For the spaces $\mathrm{SU}(n) / \mathrm{SO}(n)$ and $\mathrm{SU}(2 n) / \mathrm{USp}(n)$, the remaining coefficient $a$ can be found by evaluating the spherical functions at $e_{G}$. Thus,

$$
\begin{aligned}
& \mathrm{SU}(n) / \mathrm{SO}(n): \quad\left|\phi^{(2,0, \ldots, 0)_{n-1}}\right|^{2}=\frac{2}{n^{2}+n}+\frac{n^{2}+n-2}{n^{2}+n} \phi^{(4,2, \ldots, 2)_{n-1} ;} \\
& \mathrm{SU}(2 n) / \operatorname{USp}(n): \quad\left|\phi^{(1,1,0, \ldots, 0)_{2 n-1}}\right|^{2}=\frac{1}{2 n^{2}-n}+\frac{2 n^{2}-n-1}{2 n^{2}-n} \phi^{(2,2,1, \ldots, 1,0)_{2 n-1}} .
\end{aligned}
$$

But in the other cases, the values of the spherical functions appearing in the righthand side of the formulas of Lemma 22 are unfortunately not known $a$ priori, which makes finding the coefficients $a, b, c, \ldots$ quite difficult. However, since one only needs to compute $\mathbb{E}_{t}\left[\left(\phi^{\lambda_{\min }}\right)^{2}\right]$, and since $\phi^{\lambda_{\min }}$ is explicit in terms of matrix coefficients, one can use the following Lemma (cf. [18, Proposition 1.4]).

Lemma 23 Let $k \geq 1$ be any integer, and $\left(g_{t}\right)_{t \in \mathbb{R}_{+}}$be the Brownian motion on $\mathrm{SO}(n)$ or $\mathrm{SU}(n)$. The joint moments of order $k$ of the matrix coefficients of $g_{t}$ are given by

$$
\mathbb{E}\left[g_{t}^{\otimes k}\right]=\exp \left(t \frac{k \alpha_{\mathfrak{g}}}{2}\left(I_{n}\right)^{\otimes k}+t \sum_{1 \leq i<j \leq k} \eta_{i, j}\left(C_{\mathfrak{g}}\right)\right)
$$

where $\alpha_{\mathfrak{g}}$ is the coefficient introduced on page 10; $C_{\mathfrak{g}}$ is the Casimir operator; and $\eta_{i, j}$ is the linear map from $\mathrm{M}(n, \mathbb{k})^{\otimes 2}$ to $\mathrm{M}(n, \mathbb{k})^{\otimes k}$ defined on simple tensors $X \otimes Y$ by

$$
X \otimes Y \mapsto\left(I_{n}\right)^{\otimes(i-1)} \otimes X \otimes\left(I_{n}\right)^{\otimes(j-i-1)} \otimes Y \otimes\left(I_{n}\right)^{\otimes k-j} .
$$

In the complex case, one has also:

$$
\mathbb{E}\left[\left(g_{t}\right)^{\otimes k} \otimes\left(\overline{g_{t}}\right)^{\otimes l}\right]=\exp \left(t \frac{(k+l) \alpha_{\mathfrak{g}}}{2}\left(I_{n}\right)^{\otimes k+l}+t \sum_{1 \leq i<j \leq k+l} \tilde{\eta}_{i, j}\left(C_{\mathfrak{g}}\right)\right),
$$


with

$$
\tilde{\eta}_{i, j}(X \otimes Y)= \begin{cases}\eta_{i, j}(X \otimes Y) & \text { if } i, j \in \llbracket 1, k \rrbracket ; \\ -\eta_{i, j}\left(X \otimes Y^{t}\right) & \text { if } i \in \llbracket 1, k \rrbracket \text { and } j \in \llbracket k+1, k+l \rrbracket ; \\ \eta_{i, j}\left(X^{t} \otimes Y^{t}\right) & \text { if } i, j \in \llbracket k+1, k+l \rrbracket .\end{cases}
$$

Proof In the complex case, recall the stochastic differential equation satisfied by $g_{t}$, and therefore by $\overline{g_{t}}$ :

$$
d g_{t}=g_{t} d B_{t}+\frac{\alpha_{\mathfrak{g}}}{2} g_{t} d t \quad ; \quad d \overline{g_{t}}=-\overline{g_{t}} d B_{t}^{t}+\frac{\alpha_{\mathfrak{g}}}{2} \overline{g_{t}} d t .
$$

Itô's formula yields then a stochastic differential equation for $\left(g_{t}\right)^{\otimes k} \otimes\left(\overline{g_{t}}\right)^{\otimes l}$ :

$$
\begin{array}{r}
d\left(g^{\otimes k} \otimes \bar{g}^{\otimes l}\right)_{t}=\left(g_{t}\right)^{\otimes k} \otimes\left(\overline{g_{t}}\right)^{\otimes l}\left(\frac{(k+l) \alpha_{\mathfrak{g}}}{2} d t+\sum_{1 \leq i<j \leq k} \tilde{\eta}_{i, j}\left(d B_{t} \otimes d B_{t}\right)\right) \\
+\left(g_{t}\right)^{\otimes k} \otimes\left(\overline{g_{t}}\right)^{\otimes l}\left(\sum_{i=1}^{k}\left(I_{n}\right)^{\otimes i-1} \otimes d B_{t} \otimes\left(I_{n}\right)^{\otimes k+l-i}\right. \\
\left.-\sum_{i=k+1}^{k+l}\left(I_{n}\right)^{\otimes i-1} \otimes d B_{t}^{t} \otimes\left(I_{n}\right)^{\otimes k+l-i}\right) .
\end{array}
$$

The quadratic variation of $B_{t}$ is given by the Casimir operator: $d B_{t} \otimes d B_{t}=C_{\mathfrak{g}} d t$. Taking expectations in the formula above leads now to a differential equation for $\mathbb{E}\left[\left(g_{t}\right)^{\otimes k} \otimes\left(\overline{g_{t}}\right)^{\otimes l}\right]$, whose solution is the exponential of matrices in the statement of this lemma. The real case is the specialization $l=0$ of the previous discussion, though with a different Casimir operator. In the quaternionic case, one has to be more careful. In particular, since the quaternionic conjugate of $p q$ is $q^{\star} p^{\star}$ instead of $p^{\star} q^{\star}$, in the previous argument the SDE for $g_{t}^{\star}$ does not take the same form. A way to overcome this problem is to use the doubling map (Eq. 1.2). Thus, we write an equation for $\widetilde{g}_{t}$ instead of $g_{t}$ :

$$
\mathbb{E}\left[\left(\tilde{g}_{t}\right)^{\otimes k}\right]=\exp \left(t \frac{k \alpha_{\mathfrak{g}}}{2}\left(I_{2 n}\right)^{\otimes k}+t \sum_{1 \leq i<j \leq k} \eta_{i, j}\left(C_{\mathfrak{g}}\right)\right),
$$

where the Casimir is now considered as an element of $\left(\operatorname{End}\left(\mathbb{C}^{2 n}\right)\right)^{\otimes 2}$. As we shall see later, joint moments of the entries of $g$ and $g^{\star}$ are combinations of the joint moments of the entries of $\widetilde{g}$, so the previous formula will prove sufficient to solve our problem in the quaternionic case.

It turns out that in each case important for our computations, the matrix $\sum_{1 \leq i<j \leq k} \tilde{\eta}_{i, j}\left(C_{\mathfrak{g}}\right)$ can be explicitly diagonalized, with a basis of eigenvectors that is quite tractable (to be honest, with the help of a computer). In the following, we describe the eigenvalues and eigenvectors of these matrices, and leave the reader check that they are indeed eigenvalues and eigenvectors: this is each time an immediate computation with elementary matrices, though quite tedious if $k=4$ or $k+l=4$. For simplification, we write $e\left[i_{1}, i_{2}, \ldots, i_{r}\right]=e_{i_{1}} \otimes e_{i_{2}} \otimes \cdots \otimes e_{i_{r}}$. 


\subsubsection{Quotients of Orthogonal Groups}

For special orthogonal groups, set $\frac{1}{n} M_{n, k}=\sum_{1 \leq i<j<k} \eta_{i, j}\left(C_{\mathfrak{s o}(n)}\right)$, to be considered as an element of $\operatorname{End}\left(\left(\mathbb{R}^{n}\right)^{\otimes k}\right)$. If $k=2$, then the eigenvalues and eigenvectors of $M_{n, 2}=\sum_{1 \leq i<j \leq n}\left(E_{i j}-E_{j i}\right)^{\otimes 2}$ are:

\begin{tabular}{ccc}
\hline Eigenvalue & Multiplicity & Eigenvectors \\
\hline$n-1$ & 1 & $\sum_{i=1}^{n} e[i, i]$ \\
1 & $\frac{n(n-1)}{2}$ & $e[i, j]-e[j, i], i<j$ \\
-1 & $\frac{(n+2)(n-1)}{2}$ & $e[i, j]+e[j, i], i<j$ \\
& & $e[i, i]-e[i+1, i+1], i \leq n-1$ \\
\hline
\end{tabular}

This allows to compute $\exp \left(-\frac{(n-1) t}{n}\right) \exp \left(\frac{t}{n} M_{n, 2}\right)$, which is the right-hand side of formula 4.7 in the case of $\mathrm{SO}(n, \mathbb{R})$ and for $k=2$. One obtains:

$$
\begin{array}{ll}
\mathbb{E}\left[\left(g_{i i}\right)^{2}\right]=\frac{1}{n}+\left(1-\frac{1}{n}\right) \mathrm{e}^{-t} ; & \mathbb{E}\left[\left(g_{i j}\right)^{2}\right]=\frac{1}{n}\left(1-\mathrm{e}^{-t}\right) ; \\
\mathbb{E}\left[g_{i i} g_{j j}\right]=\frac{1}{2}\left(\mathrm{e}^{-t}+\mathrm{e}^{-\frac{n-2}{n} t}\right) \quad ; \quad \mathbb{E}\left[g_{i j} g_{j i}\right]=\frac{1}{2}\left(\mathrm{e}^{-t}-\mathrm{e}^{-\frac{n-2}{n} t}\right) ;
\end{array}
$$

and all the other mixed moments vanish (e.g., $\mathbb{E}\left[g_{i i} g_{i j}\right]$ or $\left.\mathbb{E}\left[g_{i j} g_{k l}\right]\right)$. Now, if $k=4$, then the eigenvalues and eigenvectors of $M_{n, 4}$ are:

\begin{tabular}{ccc}
\hline Eigenvalue & Multiplicity & Eigenvectors (not exhaustive, some repetitions) \\
\hline $2 n-2$ & 3 & $\sum_{k=1}^{n} \sum_{l=1}^{n} e[k, k, l, l], \star$ \\
$n$ & $3 n(n-1)$ & $\sum_{k=1}^{n} e[i, j, k, k]-e[j, i, k, k], i<j, \star$ \\
$n-2$ & $3(n+2)(n-1)$ & $\sum_{k=1}^{n} e[i, j, k, k]+e[j, i, k, k], i<j, \star$ \\
& & $\sum_{k=1}^{n} e[i, i, k, k]-e[i+1, i+1, k, k], i \leq n-1, \star$ \\
6 & $\frac{n(n-1)(n-2)(n-3)}{24}$ & $\sum_{\sigma \in \mathfrak{S}_{4}} \varepsilon(\sigma) e[i, j, k, l]^{\sigma}, i<j<k<l$ \\
2 & $\frac{3 n(n+2)(n-1)(n-3)}{8}$ & $D_{1}^{\eta}(i, j, k, l), D_{2}^{\eta}(i, j, k, l), D_{3}^{\eta}(i, j, k, l), i \neq j \neq k \neq l$ \\
0 & $\frac{n(n+1)(n+2)(n-3)}{6}$ & $S_{1}(i, j, k, l), S_{2}(i, j, k, l), i \neq j \neq k \neq l$ \\
& $\frac{3(n-1)(n-2)(n+1)(n+4)}{8}$ & $K_{1}(i, j, k, l), K_{2}(i, j, k, l), i \neq j \neq k \neq l$ \\
-2 & & $\left(\begin{array}{c}\left.e[i, j]^{\otimes 2}+e[j, k]^{\otimes 2}+e[k, i]\right]^{\otimes 2} \\
\left.-e[j, i]^{\otimes 2}-e[k, j]^{\otimes 2}-e[i, k]^{\otimes 2}\right), i \neq j \neq k, \star\end{array}\right.$ \\
& & $D_{1}^{\theta}(i, j, k, l), D_{2}^{\theta}(i, j, k, l), D_{3}^{\theta}(i, j, k, l), i \neq j \neq k \neq l$ \\
-6 & $\frac{n(n-1)(n+1)(n+6)}{24}$ & $\sum_{\sigma \in \mathfrak{S}_{4}} e[i, j, k, l]^{\sigma}, i<j<k<l$ \\
& & $e[i, i, i, i]+e[j, j, j, j]-\sum_{\sigma \in \mathfrak{S}_{4}}^{\prime} e[i, i, j, j]^{\sigma}, i<j$ \\
\hline
\end{tabular}

The star $\star$ means that the eigenvectors of a basis are listed up to action of $\mathfrak{S}_{4}$; and the symbols $\sum_{\sigma \in \mathfrak{S}_{4}}^{\prime}$ mean that we take the sum of all distinct permutations of the tensors. For the eigenvectors associated to the value 2 , denote $\mathfrak{D}_{4,(1)}=$ $\langle(1,3,2,4),(1,2)\rangle, \mathfrak{D}_{4,(2)}=\langle(1,2,3,4),(1,3)\rangle$ and $\mathfrak{D}_{4,(3)}=\langle(1,2,4,3),(1,4)\rangle$ the 
three dihedral groups of order 4 (hence cardinality 8) that can be found inside $\mathfrak{S}_{4}$. Each dihedral group of order 4 has for presentation

$$
\mathfrak{D}_{4}=\left\langle r, s \mid r^{4}=s^{2}=(r s)^{2}=1\right\rangle,
$$

so the parity $\eta(\sigma)$ of the number of occurrences of $s$ in a reduced writing of $\sigma \in \mathfrak{D}_{4}$ is well-defined, and provides a morphism $\eta: \mathfrak{D}_{4,(v)} \rightarrow\{ \pm 1\}$ for $v=1,2,3$. Then, it can be checked that for every $i \neq j \neq k \neq l$ and any $v$,

$$
D_{v}^{\eta}(i, j, k, l)=\sum_{\sigma \in \mathfrak{D}_{4,(v)}} \eta(\sigma) e[i, j, k, l]^{\sigma}
$$

is in $V_{2}$. The eigenvectors $D_{1}^{\theta}(i, j, k, l), D_{2}^{\theta}(i, j, k, l)$ and $D_{3}^{\theta}(i, j, k, l)$ associated to the eigenvalue -2 are defined exactly the same way, but with the morphism $\theta$ : $\mathfrak{D}_{4,(v)} \rightarrow\{ \pm 1\}$ associated to the parity of the number of occurrences of $r$ in a reduced decomposition of $\sigma \in \mathfrak{D}_{4}$ (again it is well defined):

$$
D_{v}^{\theta}(i, j, k, l)=\sum_{\theta \in \mathfrak{D}_{4,(v)}} \theta(\sigma) e[i, j, k, l]^{\sigma}
$$

For the eigenvectors associated to the value $0, S_{1}(i, j, k, l)$ is defined by

$$
\begin{aligned}
& e[i, i, k, k]+e[k, k, i, i]+e[j, j, l, l]+e[l, l, j, j]-e[i, i, l, l]-e[l, l, i, i]-e[j, j, k, k] \\
& \quad-e[k, k, j, j]-e[i, k, k, i]-e[k, i, i, k]-e[j, l, l, j]-e[l, j, j, l]+e[i, l, l, i] \\
& \quad+e[l, i, i, l]+e[j, k, k, j]+e[k, j, j, k]
\end{aligned}
$$

and $S_{2}(i, j, k, l)$ is obtained by replacing each term $a \otimes b \otimes b \otimes a$ by $a \otimes b \otimes a \otimes b$ in the previous formula. On the other hand, if $\mathfrak{K}_{4}=\{\mathrm{id},(1,2)(3,4),(1,3)(2,4)$, $(1,4)(2,3)\}$ denotes the Klein group, then $K_{1}(i, j, k, l)$ and $K_{2}(i, j, k, l)$ are defined as

$$
\begin{aligned}
& K_{1}(i, j, k, l)=\sum_{\sigma \in \mathfrak{K}_{4}} e[i, j, k, l]^{\sigma}-\sum_{\sigma \in(1,2,3) \mathfrak{K}_{4}} e[i, j, k, l]^{\sigma} ; \\
& K_{2}(i, j, k, l)=\sum_{\sigma \in \mathfrak{K}_{4}} e[i, j, k, l]^{\sigma}-\sum_{\sigma \in(1,3,2) \mathfrak{K}_{4}} e[i, j, k, l]^{\sigma} .
\end{aligned}
$$

That said, the deduction of the mixed moments of order 4 of the coefficients of $g$ goes as follows. One notices that

$$
\begin{aligned}
(n+2) \sum_{i=1}^{n} e_{i}^{\otimes 4}= & \left(\sum_{k, l=1}^{n} e[k, k, l, l]+e[k, l, k, l]+e[k, l, l, k]\right) \\
& +\sum_{i<j}\left(e_{i}^{\otimes 4}+e_{j}^{\otimes 4}-\sum_{\sigma \in \mathfrak{S}_{4}}^{\prime} e[i, i, j, j]^{\sigma}\right)
\end{aligned}
$$


with the first sum in the eigenspace $V_{2 n-2}$ and the second sum in the eigenspace $V_{-6}$.

On the other hand, for any $i \neq j$,

$$
\begin{aligned}
(n+4)\left(e_{i}^{\otimes 4}-e_{j}^{\otimes 4}\right)= & 6 e[i, i, i, i]+\sum_{k \neq i, j} \sum_{\sigma \in \mathfrak{S}_{4}}^{\prime} e[i, i, k, k]^{\sigma} \\
& -\sum_{k \neq i, j} \sum_{\sigma \in \mathfrak{S}_{4}}^{\prime} e[j, j, k, k]^{\sigma}-6 e[j, j, j, j] \\
& +\sum_{k \neq i, j}\left(e[i, i, i, i]+e[k, k, k, k]-\sum_{\sigma \in \mathfrak{S}_{4}}^{\prime} e[i, i, k, k]^{\sigma}\right) \\
& -\left(e[j, j, j, j]+e[k, k, k, k]-\sum_{\sigma \in \mathfrak{S}_{4}}^{\prime} e[j, j, k, k]^{\sigma}\right),
\end{aligned}
$$

with the two first lines in $V_{n-2}$ and the two last lines in $V_{-6}$. Since $e_{i}^{\otimes 4}=\frac{1}{n} \sum_{j=1}^{n} e_{j}^{\otimes 4}+$ $\frac{1}{n} \sum_{j=1}^{n}\left(e_{i}^{\otimes 4}-e_{j}^{\otimes 4}\right)$, one concludes that

$$
\begin{aligned}
e_{i}^{\otimes 4}= & \frac{1}{n(n+2)} \sum_{k, l=1}^{n} e[k, k, l, l]+e[k, l, k, l]+e[k, l, l, k] \\
& +\frac{1}{n(n+4)} \sum_{\sigma \in \mathfrak{S}_{4}}^{\prime} \sum_{k, l=1}^{n}(e[i, i, k, k]-e[l, l, k, k])^{\sigma} \\
& +\frac{n+1}{(n+2)(n+4)} \sum_{k \neq i}\left(e[i, i, i, i]+e[k, k, k, k]-\sum_{\sigma \in \mathfrak{S}_{4}}^{\prime} e[i, i, k, k]^{\sigma}\right) \\
& -\frac{1}{(n+2)(n+4)} \sum_{(k<l) \neq i}\left(e[k, k, k, k]+e[l, l, l, l]-\sum_{\sigma \in \mathfrak{S}_{4}}^{\prime} e[k, k, l, l]^{\sigma}\right),
\end{aligned}
$$

each line being in a different eigenspace: $V_{2 n-2}, V_{n-2}, V_{-6}$ and $V_{-6}$. The technique is now the following: to compute $\mathbb{E}\left[g_{i j_{1}} g_{i j_{2}} g_{i j_{3}} g_{i j_{4}}\right]$, one counts the number of occurrences of $e\left[j_{1}, j_{2}, j_{3}, j_{4}\right]$ in each term of the previous expansion. This leads to:

$$
\begin{aligned}
\mathbb{E}\left[\left(g_{i i}\right)^{4}\right] & =\frac{3}{n(n+2)}+\frac{6(n-1)}{n(n+4)} \mathrm{e}^{-t}+\frac{(n+1)(n-1)}{(n+2)(n+4)} \mathrm{e}^{-\frac{2 n+4}{n} t} ; \\
\mathbb{E}\left[\left(g_{i j}\right)^{4}\right] & =\frac{3}{n(n+2)}-\frac{6}{n(n+4)} \mathrm{e}^{-t}+\frac{3}{(n+2)(n+4)} \mathrm{e}^{-\frac{2 n+4}{n} t} ; \\
\mathbb{E}\left[\left(g_{i i}\right)^{2}\left(g_{i j}\right)^{2}\right] & =\frac{1}{n(n+2)}+\frac{(n-2)}{n(n+4)} \mathrm{e}^{-t}-\frac{(n+1)}{(n+2)(n+4)} \mathrm{e}^{-\frac{2 n+4}{n} t} ; \\
\mathbb{E}\left[\left(g_{i j}\right)^{2}\left(g_{i k}\right)^{2}\right] & =\frac{1}{n(n+2)}-\frac{2}{n(n+4)} \mathrm{e}^{-t}+\frac{1}{(n+2)(n+4)} \mathrm{e}^{-\frac{2 n+4}{n} t} ;
\end{aligned}
$$


and one sees also that the other expectations $\mathbb{E}\left[g_{i j} g_{i k} g_{i l} g_{i m}\right]$ vanish (e.g., $\mathbb{E}\left[g_{i j} g_{i k}\left(g_{i l}\right)^{2}\right]$ with $i \neq j \neq k \neq l$ ). Similar manipulations yield the decomposition in eigenvectors of $e_{i}^{\otimes 2} \otimes e_{j}^{\otimes 2}$ :

$$
\begin{aligned}
& \frac{n+1}{n(n-1)(n+2)} \sum_{k, l=1}^{n} e[k, k, l, l]-\frac{1}{(n-1) n(n+2)} \sum_{k, l=1}^{n} e[k, l, k, l]+e[k, l, l, k] \\
& +\frac{1}{n(n-2)} \sum_{k \neq i, j}\left(\sum_{l=1}^{n} e[i, i, l, l]-e[k, k, l, l]+\sum_{l=1}^{n} e[l, l, j, j]-e[l, l, k, k]\right) \\
& \quad-\frac{1}{n(n-2)(n+4)} \sum_{k \neq i, j \sigma \in \mathfrak{S}_{4}} \sum^{\prime} \\
& \quad+\left(\sum_{l=1}^{n}(e[i, i, l, l]-e[k, k, l, l])^{\sigma}+\sum_{l=1}^{n}(e[l, l, j, j]-e[l, l, k, k])^{\sigma}\right) \\
& +\frac{1}{6(n-1)(n-2)} \sum_{(k<l) \neq i, j} S_{1}(i, k, j, l)+S_{1}(i, l, j, k)+S_{2}(i, k, j, l)+S_{2}(i, l, j, k) \\
& +\frac{1}{2 n} \sum_{k \neq i, j} e[i, i, j, j]+e[j, j, k, k]+e[k, k, i, i]-e[j, j, i, i]-e[k, k, j, j] \\
& \quad-e[i, i, k, k]+\frac{1}{6(n+4)}\left(\sum_{k \neq i}\left(e_{i}^{\otimes 4}+e_{k}^{\otimes 4}-\sum_{\sigma \in \mathfrak{S}_{4}}^{\prime} e[i, i, k, k]^{\sigma}\right)^{\prime}\right. \\
& +
\end{aligned}
$$

where the eigenspaces associated to each part are $V_{2 n-2}, V_{n-2}, V_{0}, V_{-2}$ and $V_{-6}$. As a consequence,

$$
\begin{aligned}
\mathbb{E}\left[\left(g_{i i}\right)^{2}\left(g_{j j}\right)^{2}\right]= & \frac{n+1}{(n-1) n(n+2)}+\frac{2(n+3)}{n(n+4)} \mathrm{e}^{-t}+\frac{n-3}{3(n-1)} \mathrm{e}^{-\frac{2 n-2}{n} t}+\frac{n-2}{2 n} \mathrm{e}^{-2 t} \\
& +\frac{n^{2}+4 n+6}{6(n+2)(n+4)} \mathrm{e}^{-\frac{2 n+4}{n} t} ; \\
\mathbb{E}\left[\left(g_{i j}\right)^{2}\left(g_{j i}\right)^{2}\right]= & \frac{n+1}{n(n-1)(n+2)}-\frac{2}{n(n+4)} \mathrm{e}^{-t}+\frac{n-3}{3(n-1)} \mathrm{e}^{-\frac{2 n-2}{n} t}-\frac{n-2}{2 n} \mathrm{e}^{-2 t} \\
& +\frac{n^{2}+4 n+6}{6(n+2)(n+4)} \mathrm{e}^{-\frac{2 n+4}{n} t} ; \\
\mathbb{E}\left[\left(g_{i i}\right)^{2}\left(g_{j k}\right)^{2}\right]= & \frac{n+1}{n(n-1)(n+2)}+\frac{n^{2}-8}{n(n-2)(n+4)} \mathrm{e}^{-t}-\frac{n-3}{3(n-1)(n-2)} \mathrm{e}^{-\frac{2 n-2}{n} t} \\
& -\frac{1}{2 n} \mathrm{e}^{-2 t}-\frac{n}{6(n+2)(n+4)} \mathrm{e}^{-\frac{2 n+4}{n} t} ;
\end{aligned}
$$




$$
\begin{aligned}
\mathbb{E}\left[\left(g_{i j}\right)^{2}\left(g_{j k}\right)^{2}\right]= & \frac{n+1}{n(n-1)(n+2)}-\frac{2}{(n-2)(n+4)} \mathrm{e}^{-t}-\frac{n-3}{3(n-1)(n-2)} \mathrm{e}^{-\frac{2 n-2}{n} t} \\
& +\frac{1}{2 n} \mathrm{e}^{-2 t}-\frac{n}{6(n+2)(n+4)} \mathrm{e}^{-\frac{2 n+4}{n} t} ; \\
\mathbb{E}\left[\left(g_{i j}\right)^{2}\left(g_{k l}\right)^{2}\right]= & \frac{n+1}{n(n-1)(n+2)}-\frac{2(n+2)}{n(n-2)(n+4)} \mathrm{e}^{-t}+\frac{2}{3(n-1)(n-2)} \mathrm{e}^{-\frac{2 n-2}{n} t} \\
& +\frac{1}{3(n+2)(n+4)} \mathrm{e}^{-\frac{2 n+4}{n} t} ; \\
\mathbb{E}\left[g_{i i} g_{i j} g_{j j} g_{j i}\right]= & -\frac{1}{n(n-1)(n+2)}-\frac{2}{n(n+4)} \mathrm{e}^{-t}-\frac{n-3}{6(n-1)} \mathrm{e}^{-\frac{2 n-2}{n} t} \\
& +\frac{n^{2}+4 n+6}{6(n+2)(n+4)} \mathrm{e}^{-\frac{2 n+4}{n} t} ; \\
\mathbb{E}\left[g_{i k} g_{i l} g_{j k} g_{j l}\right]= & -\frac{1}{n(n-1)(n+2)}+\frac{1}{n(n-2)(n+4)} \mathrm{e}^{-t}-\frac{1}{3(n-1)(n-2)} \mathrm{e}^{-\frac{2 n-2}{n}} \\
& +\frac{1}{3(n+2)(n+4)} \mathrm{e}^{-\frac{2 n+4}{n} t} .
\end{aligned}
$$

Finally, the elementary tensor $e_{i} \otimes e_{j} \otimes e_{k} \otimes e_{l}$ with $i \neq j \neq k \neq l$ can be expanded as a combination of eigenvectors in $V_{6}, V_{2}, V_{0}, V_{-2}$ and $V_{-6}$. This expansion is related to a remarkable identity in the group algebra $\mathbb{C S}_{4}$, which can be considered as a relation of orthogonality of characters, but that only involves one-dimensional representations. Denote

$$
D_{1}^{\eta}=\sum_{\sigma \in \mathfrak{D}_{4,(1)}} \eta(\sigma) \sigma
$$

and similarly for $D_{2}^{\eta}, D_{3}^{\eta}, D_{1}^{\theta}, D_{2}^{\theta}$ and $D_{3}^{\theta}$. We also introduce $I=\sum_{\sigma \in \mathfrak{S}_{4}} \sigma, E=$ $\sum_{\sigma \in \mathfrak{S}_{4}} \varepsilon(\sigma) \sigma$, and

$$
K_{1}=\sum_{\sigma \in \mathfrak{K}_{4}} \sigma-\sum_{\sigma \in(1,2,3) \mathfrak{K}_{4}} \sigma \quad ; \quad K_{2}=\sum_{\sigma \in \mathfrak{K}_{4}} \sigma-\sum_{\sigma \in(1,3,2) \mathfrak{K}_{4}} \sigma .
$$

As explained before, all these sums correspond to eigenvectors in $V_{6}, V_{2}, V_{0}, V_{-2}$ and $V_{-6}$. Then,

$$
\mathrm{id}_{\llbracket 1,4 \rrbracket}=\frac{1}{24} I+\frac{1}{8}\left(D_{1}^{\eta}+D_{2}^{\eta}+D_{3}^{\eta}\right)+\frac{1}{12}\left(K_{1}+K_{2}\right)+\frac{1}{8}\left(D_{1}^{\theta}+D_{2}^{\theta}+D_{3}^{\theta}\right)+\frac{1}{24} E .
$$

As a consequence, $e[i, j, k, l]$ is equal to

$$
\begin{aligned}
& \frac{1}{24} \sum_{\sigma \in \mathfrak{S}_{4}} \varepsilon(\sigma) e[i, j, k, l]^{\sigma}+\frac{1}{8}\left(D_{1}^{\eta}(i, j, k, l)+D_{2}^{\eta}(i, j, k, l)+D_{3}^{\eta}(i, j, k, l)\right) \\
& +\frac{1}{12}\left(K_{1}(i, j, k, l)+K_{2}(i, j, k, l)\right)+\frac{1}{8}\left(D_{1}^{\theta}(i, j, k, l)+D_{2}^{\theta}(i, j, k, l)+D_{3}^{\theta}(i, j, k, l)\right) \\
& +\frac{1}{24} \sum_{\sigma \in \mathfrak{S}_{4}} e[i, j, k, l]^{\sigma}
\end{aligned}
$$


with each term respectively in $V_{6}, V_{2}, V_{0}, V_{-2}$ and $V_{-6}$. This leads to

$$
\begin{aligned}
& \mathbb{E}\left[g_{i i} g_{j j} g_{k k} g_{l l}\right]=\frac{1}{24} \mathrm{e}^{-\frac{2 n-8}{n} t}+\frac{3}{8} \mathrm{e}^{-\frac{2 n-4}{n} t}+\frac{1}{6} \mathrm{e}^{-\frac{2 n-2}{n} t}+\frac{3}{8} \mathrm{e}^{-2 t}+\frac{1}{24} \mathrm{e}^{-\frac{2 n+4}{n} t} ; \\
& \mathbb{E}\left[g_{i j} g_{j k} g_{k l} g_{l i}\right]=-\frac{1}{24} \mathrm{e}^{-\frac{2 n-8}{n} t}+\frac{1}{8} \mathrm{e}^{-\frac{2 n-4}{n} t}-\frac{1}{8} \mathrm{e}^{-2 t}+\frac{1}{24} \mathrm{e}^{-\frac{2 n+4}{n} t} ; \\
& \mathbb{E}\left[g_{i i} g_{j j} g_{k l} g_{l k}\right]=-\frac{1}{24} \mathrm{e}^{-\frac{2 n-8}{n} t}-\frac{1}{8} \mathrm{e}^{-\frac{2 n-4}{n} t}+\frac{1}{8} \mathrm{e}^{-2 t}+\frac{1}{24} \mathrm{e}^{-\frac{2 n+4}{n} t} ; \\
& \mathbb{E}\left[g_{i j} g_{j i} g_{k l} g_{l k}\right]=\frac{1}{24} \mathrm{e}^{-\frac{2 n-8}{n} t}-\frac{1}{8} \mathrm{e}^{-\frac{2 n-4}{n} t}+\frac{1}{6} \mathrm{e}^{-\frac{2 n-2}{n} t}-\frac{1}{8} \mathrm{e}^{-2 t}+\frac{1}{24} \mathrm{e}^{-\frac{2 n+4}{n} t} ;
\end{aligned}
$$

and we are done with the computations in the case of special orthogonal groups.

Proposition 24 For the real Grassmannian varieties $\operatorname{Gr}(n, q, \mathbb{R})$ and the spaces $\mathrm{SO}(2 n) / \mathrm{U}(n)$, the coefficients of Lemma 22 are:

$$
\begin{aligned}
\operatorname{Gr}(n, q, \mathbb{R}): \quad & \frac{2}{n^{2}+n-2}+\frac{2 n^{2}}{3}\left(\frac{1}{(n-1)(n-2)}-\frac{1}{p q(n-2)}\right) \phi^{(2,2,0, \ldots, 0)\left\lfloor\frac{n}{2}\right\rfloor} \\
& +\frac{\frac{4 n^{2}}{p q}-16}{(n-2)(n+4)} \phi^{(2,0, \ldots, 0)_{\left\lfloor\frac{n}{2}\right\rfloor}} \\
+ & \frac{n^{2}}{3}\left(\frac{1}{(n+2)(n+4)}+\frac{2}{p q(n+4)}\right) \phi^{(4,0, \ldots, 0)_{\left\llcorner\frac{n}{2}\right\rfloor}} \\
\mathrm{SO}(2 n) / \mathrm{U}(n): & \frac{1}{2 n^{2}-n}+\frac{n-1}{3 n} \phi^{\left(1^{4}, 0, \ldots, 0\right)_{n}}+\frac{4\left(n^{2}-1\right)}{(3 n)(2 n-1)} \phi^{(2,2,0, \ldots, 0)_{n}} .
\end{aligned}
$$

Proof One expands the square of the sum given by Proposition 21, and one gathers the joint moments of the coefficients according to the possible identities between the indices. For real Grassmannians, $\left(\phi^{(2,0, \ldots, 0)\left\lfloor\frac{n}{2}\right\rfloor}\right)^{2}$ has for expansion:

$$
\begin{aligned}
\left(\frac{1}{p} \sum_{i, j \leq p}\left(g_{i j}\right)^{2}+\frac{1}{q} \sum_{i, j>p}\left(g_{i j}\right)^{2}-1\right)^{2}= & \left(\frac{1}{p} \sum_{i, j \leq p}\left(g_{i j}\right)^{2}+\frac{1}{q} \sum_{i, j>p}\left(g_{i j}\right)^{2}\right)^{2}-2 \phi^{(2,0, \ldots, 0)\left\lfloor\frac{n}{2}\right\rfloor}-1 \\
= & \left(\frac{n}{p q}\right) T\left[\left(g_{11}\right)^{4}\right]+\left(4-\frac{n}{p q}\right) T\left[\left(g_{11} g_{22}\right)^{2}\right] \\
& +\left(2-\frac{n}{p q}\right)\left(4 T\left[\left(g_{11} g_{12}\right)^{2}\right]+T\left[\left(g_{12}\right)^{4}\right]\right. \\
& \left.+T\left[\left(g_{12} g_{21}\right)^{2}\right]\right)+\left(4 n-16+\frac{4 n}{p q}\right) T\left[\left(g_{11} g_{23}\right)^{2}\right] \\
& +\left(2 n-12+\frac{4 n}{p q}\right)\left(T\left[\left(g_{12} g_{13}\right)^{2}\right]+T\left[\left(g_{12} g_{23}\right)^{2}\right]\right) \\
& +\left(n^{2}-8 n+24-\frac{6 n}{p q}\right) T\left[\left(g_{12} g_{34}\right)^{2}\right] \\
& -2 \phi^{(2,0, \ldots, 0)\left\lfloor\frac{n}{2}\right\rfloor}-1 .
\end{aligned}
$$


where by $T\left[\left(g_{11}\right)^{4}\right]$ we mean a linear combination of products $\left(g_{i i}\right)^{4}$, whose expectation is therefore $\mathbb{E}\left[\left(g_{11}\right)^{4}\right]$; by $T\left[\left(g_{11} g_{22}\right)^{2}\right]$ we mean a linear combination of products $\left(g_{i i} g_{j j}\right)^{2}$ whose expectation will be $\mathbb{E}\left[\left(g_{11} g_{22}\right)^{2}\right]$, etc. Thus, the expectation of $\left(\phi^{(2,0, \ldots, 0)\left\lfloor\frac{n}{2}\right\rfloor}\right)^{2}$ is

$$
\begin{aligned}
\frac{2}{n^{2}+n-2} & +\frac{\frac{4 n^{2}}{p q}-16}{(n-2)(n+4)} \mathrm{e}^{-t}+\frac{2 n^{2}}{3}\left(\frac{1}{(n-1)(n-2)}-\frac{1}{p q(n-2)}\right) \mathrm{e}^{-\frac{2 n-2}{n} t} \\
& +\frac{n^{2}}{3}\left(\frac{1}{(n+2)(n+4)}+\frac{2}{p q(n+4)}\right) \mathrm{e}^{-\frac{2 n+4}{n} t}
\end{aligned}
$$

and by identifying the Casimir coefficients of the spherical functions, one deduces from this the expansion of the square of the discriminating zonal function in zonal functions.

For the spaces $\mathrm{SO}(2 n) / \mathrm{U}(n)$, one computes again the square of the homogeneous polynomial of degree 2 given by Proposition 21 . Thus, $\frac{1}{n^{2}}\left(\sum_{i, j=1}^{n} g_{2 i, 2 j} g_{2 i-1,2 j-1}-\right.$ $\left.g_{2 i, 2 j-1} g_{2 i-1,2 j}\right)^{2}$ equals

$$
\begin{aligned}
& \frac{1}{n}\left(T\left[\left(g_{11} g_{22}\right)^{2}\right]+T\left[\left(g_{12} g_{21}\right)^{2}\right]-2 T\left[g_{11} g_{12} g_{22} g_{21}\right]\right) \\
& \quad+\frac{n-1}{n}\left(2 T\left[\left(g_{12} g_{34}\right)^{2}\right]-2 T\left[g_{13} g_{14} g_{23} g_{24}\right]\right) \\
& \quad+\frac{n-1}{n}\left(2 T\left[g_{12} g_{21} g_{34} g_{43}\right]-2 T\left[g_{12} g_{23} g_{34} g_{41}\right]\right) \\
& \quad+\frac{n-1}{n}\left(T\left[g_{11} g_{22} g_{33} g_{44}\right]+T\left[g_{12} g_{21} g_{34} g_{43}\right]-2 T\left[g_{11} g_{22} g_{34} g_{43}\right]\right) \\
& \quad+\text { remainder, }
\end{aligned}
$$

with the same notations as before, and where the remainder is a combination of products of coefficients whose expectation vanish under Brownian (and Haar) measures. More precisely, terms with a certain symmetry cancel with one another when taking the expectation: for instance,

$$
\left(g_{2 i, 2 j} g_{2 i-1,2 j-1}-g_{2 i, 2 j-1} g_{2 i-1,2 j}\right) \times\left(g_{2 k, 2 l} g_{2 k-1,2 l-1}-g_{2 k, 2 l-1} g_{2 k-1,2 l}\right)
$$

with $i \neq j \neq k \neq l$ is equal to $a+b-c-d$, where $a, b, c, d$ are products of type $g_{i j} g_{k l} g_{m n} g_{o p}$, and have therefore the same expectation. Consequently, every product of type (Eq. 4.8) will not contribute to the expectation of $\left(\phi^{(1,1,0, \ldots, 0)\left\lfloor\frac{n}{2}\right\rfloor}\right)^{2}$. The following sets of indices have the same property of "self-cancellation":

$$
\begin{aligned}
& (i, i, i, j) ;(i, i, j, i) ;(i, j, i, i) ;(j, i, i, i) ;(i, i, j, k) ;(j, k, i, i) ; \\
& (i, j, i, k) ;(j, i, k, i) ;(i, j, k, i) ;(j, i, i, k) ;(i, j, k, l) ;(i, j, k, l) ;
\end{aligned}
$$

so it suffices to consider products with sets of indices $(i, i, i, i),(i, j, i, j),(i, j, j, i)$ or $(i, i, j, j)$ - these are the four lines of the previous expansion. Using the formulas given 
before for the joint moments of the entries (beware that one has to use them with the parameter $2 n$ ), we obtain:

$$
\mathbb{E}\left[\left(\phi^{(1,1,0, \ldots, 0)_{n}}\right)^{2}\right]=\frac{1}{n(2 n-1)}+\frac{n-1}{3 n} \mathrm{e}^{-\frac{2 n-4}{n} t}+\frac{4\left(n^{2}-1\right)}{(3 n)(2 n-1)} \mathrm{e}^{-\frac{2 n-1}{n} t}
$$

and it suffices then to identify the coefficients of the negative exponentials.

\subsubsection{Quotients of Unitary Groups}

For special unitary groups, set $\frac{1}{n^{2}} M_{n, k, l}=\sum_{1 \leq i<j \leq k+l} \tilde{\eta}_{i, j}\left(C_{\mathfrak{s u}(n)}\right)$, to be considered as an element of $\operatorname{End}\left(\left(\mathbb{C}^{n}\right)^{\otimes k+l}\right)$. If $k=l=1$, then

$$
\mathbb{E}\left[\left|g_{i i}\right|^{2}\right]=\frac{1}{n}+\left(1-\frac{1}{n}\right) \mathrm{e}^{-t} \quad ; \quad \mathbb{E}\left[\left|g_{i j}\right|^{2}\right]=\frac{1}{n}\left(1-\mathrm{e}^{-t}\right) \quad ; \quad \mathbb{E}\left[g_{i i} \overline{g_{j j}}\right]=\mathrm{e}^{-t}
$$

since the eigenvalues and eigenvectors of $M_{n, 1,1}=\mathrm{i} I_{n} \otimes \mathrm{i} I_{n}+n \sum_{i, j=1}^{n} E_{i j} \otimes E_{i j}$ are:

\begin{tabular}{ccc}
\hline Eigenvalue & Multiplicity & Eigenvectors \\
\hline$n^{2}-1$ & 1 & $\sum_{i=1}^{n} e[i, i]$ \\
-1 & $n^{2}-1$ & $e[i, j], 1 \leq i \neq j \leq n$ \\
& & $e[i, i]-e[i+1, i+1], i \leq n-1$ \\
\hline
\end{tabular}

If $k=l=2$, then the eigenvalues and eigenvectors of $M_{n, 2,2}$ are:

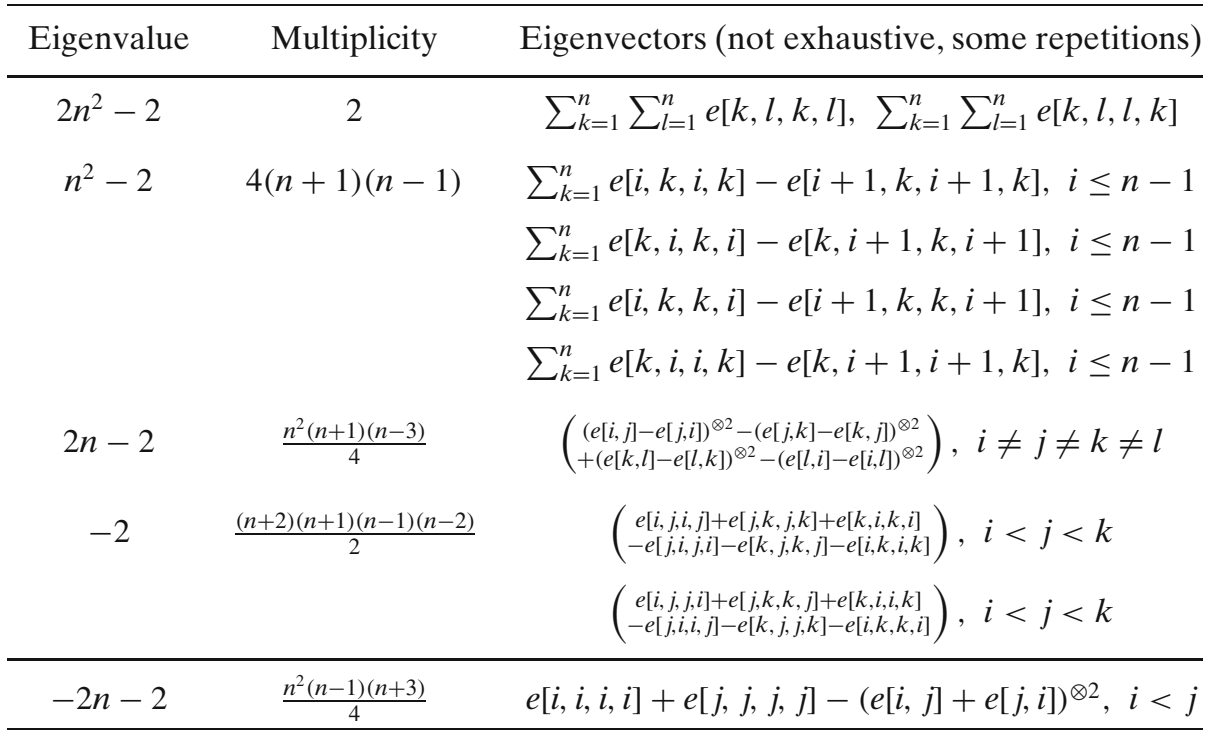


Again, we can use the previous table to decompose some elementary 4-tensors in eigenvectors of $M_{n, 2,2}$; we refer to Appendix 5.4. Thus,

$$
\begin{aligned}
& \mathbb{E}\left[\left|g_{i i}\right|^{4}\right]=\frac{2}{n(n+1)}+\frac{4(n-1)}{n(n+2)} \mathrm{e}^{-t}+\frac{n(n-1)}{(n+1)(n+2)} \mathrm{e}^{-\frac{2 n+2}{n} t} \\
& \mathbb{E}\left[\left|g_{i j}\right|^{4}\right]=\frac{2}{n(n+1)}-\frac{4}{n(n+2)} \mathrm{e}^{-t}+\frac{2}{(n+1)(n+2)} \mathrm{e}^{-\frac{2 n+2}{n} t}
\end{aligned}
$$

$\mathbb{E}\left[\left|g_{i i}\right|^{2}\left|g_{i j}\right|^{2}\right]=\frac{1}{n(n+1)}+\frac{n-2}{n(n+2)} \mathrm{e}^{-t}-\frac{n}{(n+1)(n+2)} \mathrm{e}^{-\frac{2 n+2}{n} t}$

$\mathbb{E}\left[\left|g_{i j}\right|^{2}\left|g_{i k}\right|^{2}\right]=\frac{1}{n(n+1)}-\frac{2}{n(n+2)} \mathrm{e}^{-t}+\frac{1}{(n+1)(n+2)} \mathrm{e}^{-\frac{2 n+2}{n} t}$

$$
\begin{aligned}
\mathbb{E}\left[\left|g_{i i}\right|^{2}\left|g_{j j}\right|^{2}\right]= & \frac{1}{(n-1)(n+1)}+\frac{2(n+1)}{n(n+2)} \mathrm{e}^{-t}+\frac{n-3}{4(n-1)} \mathrm{e}^{-\frac{2 n-2}{n} t} \\
& +\frac{n-2}{2 n} \mathrm{e}^{-2 t}+\frac{n^{2}+n+2}{4(n+1)(n+2)} \mathrm{e}^{-\frac{2 n+2}{n} t}
\end{aligned}
$$

$\mathbb{E}\left[\left|g_{i j}\right|^{2}\left|g_{j i}\right|^{2}\right]=\frac{1}{(n-1)(n+1)}-\frac{2}{n(n+2)} \mathrm{e}^{-t}+\frac{n-3}{4(n-1)} \mathrm{e}^{-\frac{2 n-2}{n} t}$

$$
-\frac{n-2}{2 n} \mathrm{e}^{-2 t}+\frac{n^{2}+n+2}{4(n+1)(n+2)} \mathrm{e}^{-\frac{2 n+2}{n} t}
$$

$\mathbb{E}\left[\left|g_{i i}\right|^{2}\left|g_{j k}\right|^{2}\right]=\frac{1}{(n-1)(n+1)}+\frac{n^{2}-2 n-2}{n(n-2)(n+2)} \mathrm{e}^{-t}-\frac{n-3}{4(n-1)(n-2)} \mathrm{e}^{-\frac{2 n-2}{n} t}$

$$
-\frac{1}{2 n} \mathrm{e}^{-2 t}-\frac{n-1}{4(n+1)(n+2)} \mathrm{e}^{-\frac{2 n+2}{n} t}
$$

$\mathbb{E}\left[\left|g_{i j}\right|^{2}\left|g_{j k}\right|^{2}\right]=\frac{1}{(n-1)(n+1)}-\frac{2(n-1)}{n(n-2)(n+2)} \mathrm{e}^{-t}-\frac{n-3}{4(n-1)(n-2)} \mathrm{e}^{-\frac{2 n-2}{n} t}$

$$
+\frac{1}{2 n} \mathrm{e}^{-2 t}-\frac{n-1}{4(n+1)(n+2)} \mathrm{e}^{-\frac{2 n+2}{n} t}
$$

$\mathbb{E}\left[\left|g_{i j}\right|^{2}\left|g_{k l}\right|^{2}\right]=\frac{1}{(n-1)(n+1)}-\frac{2}{(n-2)(n+2)} \mathrm{e}^{-t}+\frac{1}{2(n-1)(n-2)} \mathrm{e}^{-\frac{2 n-2}{n} t}$

$$
+\frac{1}{2(n+1)(n+2)} \mathrm{e}^{-\frac{2 n+2}{n} t}
$$


Proposition 25 For the symmetric spaces with isometry group $\mathrm{SU}(n)$ or $\mathrm{SU}(2 n)$, the coefficients of Lemma 22 are:

$$
\begin{aligned}
& \operatorname{Gr}(n, q, \mathbb{C}): \frac{1}{n^{2}-1}+\frac{n^{2}}{2}\left(\frac{\frac{2 n^{2}}{p q}-8}{n^{2}-4} \phi^{(2,1, \ldots, 1)_{n-1}} \frac{1}{(n-1)(n-2)}-\frac{1}{p q(n-2)}\right) \phi^{(2,2,1, \ldots, 1,0)_{n-1} ;} \\
&+\frac{n^{2}}{2}\left(\frac{1}{(n+1)(n+2)}+\frac{1}{p q(n+2)}\right) \phi^{(4,2, \ldots, 2)_{n-1} ;} \\
& \mathrm{SU}(n) / \mathrm{SO}(n): \quad \frac{2}{n^{2}+n}+\frac{n^{2}+n-2}{n^{2}+n} \phi^{(4,2, \ldots, 2)_{n-1}} ;
\end{aligned}
$$$$
\operatorname{SU}(2 n) / \operatorname{USp}(n): \quad \frac{1}{2 n^{2}-n}+\frac{2 n^{2}-n-1}{2 n^{2}-n} \phi^{(2,2,1, \ldots, 1,0)_{2 n-1}} \text {. }
$$

Proof For $\mathrm{SU}(n) / \mathrm{SO}(n)$ and $\mathrm{SU}(2 n) / \mathrm{USp}(n)$, the only missing coefficient has already been computed. For complex Grassmannians, $\left(\phi^{(2,1, \ldots, 1)_{n-1}}\right)^{2}$ has exactly the same expansion as in the real case, but with square modules. From the computation of the joint moments $\mathbb{E}\left[\left|g_{i j} g_{k l}\right|^{2}\right]$ performed previously, one deduces that the expectation of the square of the discriminating zonal function is

$$
\begin{aligned}
\frac{1}{n^{2}-1} & +\frac{\frac{2 n^{2}}{p q}-8}{n^{2}-4} \mathrm{e}^{-t}+\frac{n^{2}}{2}\left(\frac{1}{(n-1)(n-2)}-\frac{1}{p q(n-2)}\right) \mathrm{e}^{-\frac{2 n-2}{n} t} \\
& +\frac{n^{2}}{2}\left(\frac{1}{(n+1)(n+2)}+\frac{1}{p q(n+2)}\right) \mathrm{e}^{-\frac{2 n+2}{n} t}
\end{aligned}
$$

whence the expansion in zonal spherical functions by identifying the coefficients.

\subsubsection{Quotients of Symplectic Groups}

Finally, set $\frac{1}{n} M_{n, k}=\sum_{1 \leq i<j \leq k+l} \widetilde{\eta}_{i, j}\left(C_{\mathfrak{u s p}(n)}\right)$, which is considered as an element of End $\left(\left(\mathbb{C}^{2 n}\right)^{\otimes k}\right)$. Recall that the diagonalization of these matrices will yield the joint moments of the entries of $\widetilde{g}$, the matrix obtained from $g$ by the map (Eq. 1.2). Again, as a warm-up, let us compute the joint moments of order 2 . If $k=2$, then

$$
\begin{aligned}
& \mathbb{E}\left[\left|g_{i i}\right|^{2}\right]=\frac{1}{n}+\frac{n-1}{n} \mathrm{e}^{-t} \quad ; \quad \mathbb{E}\left[\left|g_{i j}\right|^{2}\right]=\frac{1}{n}\left(1-\mathrm{e}^{-t}\right) \quad \forall i, j \in \llbracket 1, n \rrbracket ; \\
& \mathbb{E}\left[\left(\widetilde{g}_{i i}\right)^{2}\right]=\mathrm{e}^{-\frac{n+1}{n} t} \quad ; \quad \mathbb{E}\left[\left(\widetilde{g}_{i j}\right)^{2}\right]=0 \quad \forall i, j \in \llbracket 1,2 n \rrbracket ;
\end{aligned}
$$

since the eigenvectors and eigenvalues of $M_{n, 2}$ are:

\begin{tabular}{ccc}
\hline Eigenvalue & Multiplicity & Eigenvectors \\
\hline$\frac{2 n+1}{2}$ & 1 & $\sum_{i=1}^{n} e[2 i-1,2 i]-e[2 i, 2 i-1]$ \\
$\frac{1}{2}$ & $(n-1)(2 n+1)$ & $(e[2 i-1,2 i]-e[2 i, 2 i-1])-(e[2 i+1,2 i+2]-e[2 i+2,2 i+1]), i \leq n-1$ \\
& & $e[2 i-1,2 j-1]-e[2 j-1,2 i-1], e[2 i, 2 j]-e[2 j, 2 i], 1 \leq i<j \leq n$ \\
& & $e[2 i-1,2 j]-e[2 j, 2 i-1], 1 \leq i \neq j \leq n$ \\
$-\frac{1}{2}$ & $n(2 n+1)$ & $e_{k} \otimes e_{l}+e_{l} \otimes e_{k}, 1 \leq k \leq l \leq 2 n$ \\
\hline
\end{tabular}


For $k=4$, we refer to Appendix 5.5 for the expansion in eigenvectors of simple tensors. One obtains:

$$
\begin{aligned}
\mathbb{E}\left[\left(\widetilde{g}_{i i}\right)^{4}\right]= & \mathrm{e}^{-\frac{2 n+4}{n} t} ; \\
\mathbb{E}\left[\left(\widetilde{g}_{i j}\right)^{4}\right]= & \mathbb{E}\left[\left(\widetilde{g}_{i i} \widetilde{g}_{i j}\right)^{2}\right]=\mathbb{E}\left[\left(\widetilde{g}_{i j} \widetilde{g}_{i k}\right)^{2}\right]=0 \\
\mathbb{E}\left[\left(\widetilde{g}_{2 i-1,2 i-1} \widetilde{g}_{2 i, 2 i}\right)^{2}\right]= & \frac{1}{n(2 n+1)}+\frac{n-1}{n(n+1)} \mathrm{e}^{-t}+\frac{1}{n+1} \mathrm{e}^{-\frac{n+1}{n} t} \\
& +\frac{(2 n-1)(2 n-2)}{3(2 n+1)(2 n+2)} \mathrm{e}^{-\frac{2 n+1}{n} t}+\frac{n-1}{2(n+1)} \mathrm{e}^{-\frac{2 n+2}{n} t}+\frac{1}{6} \mathrm{e}^{-\frac{2 n+4}{n} t} ; \\
\mathbb{E}\left[\left(\widetilde{g}_{2 i-1,2 i} \widetilde{g}_{2 i, 2 i-1}\right)^{2}\right]= & \frac{1}{n(2 n+1)}+\frac{n-1}{n(n+1)} \mathrm{e}^{-t}-\frac{1}{n+1} \mathrm{e}^{-\frac{n+1}{n} t} \\
& +\frac{(2 n-1)(2 n-2)}{3(2 n+1)(2 n+2)} \mathrm{e}^{-\frac{2 n+1}{n} t}-\frac{n-1}{2(n+1)} \mathrm{e}^{-\frac{2 n+2}{n} t}+\frac{1}{6} \mathrm{e}^{-\frac{2 n+4}{n} t} ; \\
\mathbb{E}\left[\left(\widetilde{g}_{2 i-1,2 j-1} \widetilde{g}_{2 i, 2 j}\right)^{2}\right]= & \mathbb{E}\left[\left(\widetilde{g}_{2 i-1,2 j} \widetilde{g}_{2 i, 2 j-1}\right)^{2}\right]=\frac{1}{n(2 n+1)}-\frac{1}{n(n+1)} \mathrm{e}^{-t} \\
& +\frac{1}{(2 n+1)(n+1)} \mathrm{e}^{-\frac{2 n+1}{n} t} ;
\end{aligned}
$$

and the other moments of type $\mathbb{E}\left[\left(\widetilde{g}_{2 i-1, a} \widetilde{g}_{2 i, b}\right)^{2}\right]$ vanish. On the other hand, assuming that $\{a, b\}$ is not a pair $\{2 i-1,2 i\}$ in $\llbracket 1,2 n \rrbracket$, one has also

$$
\begin{aligned}
& \mathbb{E}\left[\left(\widetilde{g}_{a a} \widetilde{g}_{b b}\right)^{2}\right]=\frac{1}{3} \mathrm{e}^{-\frac{2 n+1}{n} t}+\frac{1}{2} \mathrm{e}^{-\frac{2 n+2}{n} t}+\frac{1}{6} \mathrm{e}^{-\frac{2 n+4}{n} t} ; \\
& \mathbb{E}\left[\left(\widetilde{g}_{a b} \widetilde{g}_{b a}\right)^{2}\right]=\frac{1}{3} \mathrm{e}^{-\frac{2 n+1}{n} t}-\frac{1}{2} \mathrm{e}^{-\frac{2 n+2}{n} t}+\frac{1}{6} \mathrm{e}^{-\frac{2 n+4}{n} t} ;
\end{aligned}
$$

and the other moments of type $\mathbb{E}\left[\left(\widetilde{g}_{a b} \widetilde{g}_{c d}\right)^{2}\right]$ with $\{c, d\} \neq\{a, b\}$ vanish.

The same expansions enable one to compute many moments of type $\mathbb{E}\left[\left|g_{i j} g_{k l}\right|^{2}\right]$, namely, all those that write as $\mathbb{E}\left[\left|g_{i j} g_{i k}\right|^{2}\right]$. For instance, since $\left|g_{i i}\right|^{4}=\left(\widetilde{g}_{2 i-1,2 i-1} \widetilde{g}_{2 i, 2 i}-\right.$ $\left.\widetilde{g}_{2 i-1,2 i} \widetilde{g}_{2 i, 2 i-1}\right)^{2}$, its expectation is a combination of those of $\left(\widetilde{g}_{2 i-1,2 i-1} \widetilde{g}_{2 i, 2 i}\right)^{2}$, $\left(\widetilde{g}_{2 i-1,2 i} \widetilde{g}_{2 i, 2 i-1}\right)^{2}$ and $\widetilde{g}_{2 i-1,2 i-1} \widetilde{g}_{2 i-1,2 i} \widetilde{g}_{2 i, 2 i} \widetilde{g}_{2 i, 2 i-1}$. This last expectation is

$$
-\frac{1}{2 n(2 n+1)}-\frac{n-1}{2 n(n+1)} \mathrm{e}^{-t}-\frac{(2 n-1)(2 n-2)}{6(2 n+1)(2 n+2)} \mathrm{e}^{-\frac{2 n+1}{n} t}+\frac{1}{6} \mathrm{e}^{-\frac{2 n+4}{n} t}
$$

Thus, with a few more computations, one gets

$$
\begin{aligned}
& \mathbb{E}\left[\left|g_{i i}\right|^{4}\right]=\frac{3}{n(2 n+1)}+\frac{3(n-1)}{n(n+1)} \mathrm{e}^{-t}+\frac{(2 n-1)(2 n-2)}{(2 n+1)(2 n+2)} \mathrm{e}^{-\frac{2 n+1}{n} t} ; \\
& \mathbb{E}\left[\left|g_{i j}\right|^{4}\right]=\frac{3}{n(2 n+1)}-\frac{3}{n(n+1)} \mathrm{e}^{-t}+\frac{3}{(2 n+1)(n+1)} \mathrm{e}^{-\frac{2 n+1}{n} t}
\end{aligned}
$$


$\mathbb{E}\left[\left|g_{i i} g_{i j}\right|^{2}\right]=\frac{2}{n(2 n+1)}+\frac{(n-2)}{n(n+1)} \mathrm{e}^{-t}-\frac{2(2 n-1)}{(2 n+1)(2 n+2)} \mathrm{e}^{-\frac{2 n+1}{n} t} ;$

$\mathbb{E}\left[\left|g_{i j} g_{i k}\right|^{2}\right]=\frac{2}{n(2 n+1)}-\frac{2}{n(n+1)} \mathrm{e}^{-t}+\frac{2}{(2 n+1)(n+1)} \mathrm{e}^{-\frac{2 n+1}{n} t} ;$

$\mathbb{E}\left[\left|g_{i i} g_{j j}\right|^{2}\right]=\frac{2 n-1}{n(n-1)(2 n+1)}+\frac{2}{n+1} \mathrm{e}^{-t}+\frac{n-3}{6(n-1)} \mathrm{e}^{-\frac{2 n-2}{n} t}$

$$
+\frac{n-2}{2 n} \mathrm{e}^{-2 t}+\frac{2 n^{2}-n+3}{3(n+1)(2 n+1)} \mathrm{e}^{-\frac{2 n+1}{n} t} ;
$$

$\mathbb{E}\left[\left|g_{i j} g_{i j}\right|^{2}\right]=\frac{2 n-1}{n(n-1)(2 n+1)}-\frac{2}{n(n+1)} \mathrm{e}^{-t}+\frac{n-3}{6(n-1)} \mathrm{e}^{-\frac{2 n-2}{n} t}$

$$
-\frac{n-2}{2 n} \mathrm{e}^{-2 t}+\frac{2 n^{2}-n+3}{3(n+1)(2 n+1)} \mathrm{e}^{-\frac{2 n+1}{n} t}
$$

$\mathbb{E}\left[\left|g_{i i} g_{j k}\right|^{2}\right]=\frac{2 n-1}{n(n-1)(2 n+1)}+\frac{n^{2}-3 n+1}{n(n+1)(n-2)} \mathrm{e}^{-t}-\frac{n-3}{6(n-1)(n-2)} \mathrm{e}^{-\frac{2 n-2}{n} t}$

$$
-\frac{1}{2 n} \mathrm{e}^{-2 t}-\frac{2 n-3}{3(n+1)(2 n+1)} \mathrm{e}^{-\frac{2 n+1}{n} t}
$$

$\mathbb{E}\left[\left|g_{i j} g_{j k}\right|^{2}\right]=\frac{2 n-1}{n(n-1)(2 n+1)}-\frac{2 n-3}{n(n+1)(n-2)} \mathrm{e}^{-t}-\frac{n-3}{6(n-1)(n-2)} \mathrm{e}^{-\frac{2 n-2}{n} t}$

$$
+\frac{1}{2 n} \mathrm{e}^{-2 t}-\frac{2 n-3}{3(n+1)(2 n+1)} \mathrm{e}^{-\frac{2 n+1}{n} t} \text {; }
$$

$\mathbb{E}\left[\left|g_{i j} g_{k l}\right|^{2}\right]=\frac{2 n-1}{n(n-1)(2 n+1)}-\frac{2 n-2}{n(n+1)(n-2)} \mathrm{e}^{-t}+\frac{1}{3(n-1)(n-2)} \mathrm{e}^{-\frac{2 n-2}{n} t}$

$$
+\frac{4}{3(n+1)(2 n+1)} \mathrm{e}^{-\frac{2 n+1}{n} t} .
$$

Proposition 26 For the quaternionic Grassmannian varieties $\operatorname{Gr}(n, q, \mathbb{H})$ and the spaces $\mathrm{USp}(n) / \mathrm{U}(n)$, the coefficients of Lemma 22 are:

$$
\begin{aligned}
\operatorname{Gr}(n, q, \mathbb{H}): & \frac{1}{2 n^{2}-n-1}+\frac{n^{2}}{3}\left(\frac{1}{(n-1)(n-2)}-\frac{1}{p q(n-2)}\right) \phi^{\left(1^{4}, 0, \ldots, 0\right)_{n}} \\
& +\frac{\frac{n^{2}}{p q}-4}{(n-2)(n+1)} \phi^{\left(1^{2}, 0, \ldots, 0\right)_{n-1}} \\
& +\frac{n^{2}}{3}\left(\frac{4}{(n+1)(2 n+1)}+\frac{1}{p q(n+1)}\right) \phi^{(2,2,0, \ldots, 0)_{n}} \\
\operatorname{USp}(n) / \mathrm{U}(n): & \frac{1}{2 n^{2}+n}+\frac{4(n-1)(n+1)}{3 n(2 n+1)} \phi^{(2,2,0, \ldots, 0)_{n}}+\frac{n+1}{3 n} \phi^{(4,0, \ldots, 0)_{n}} .
\end{aligned}
$$


Proof The case of quaternionic Grassmannians is again done by using the expansion on page 62 , with square modules instead of squares. One obtains the following formula for the expectation of $\left(\phi^{(1,1,0, \ldots, 0)_{n}}\right)^{2}$ :

$$
\begin{aligned}
\frac{1}{2 n^{2}-n-1} & +\frac{\frac{n^{2}}{p q}-4}{(n-2)(n+1)} \mathrm{e}^{-t}+\frac{n^{2}}{3}\left(\frac{1}{(n-1)(n-2)}-\frac{1}{p q(n-2)}\right) \mathrm{e}^{-\frac{2 n-2}{n} t} \\
& +\frac{n^{2}}{3}\left(\frac{4}{(n+1)(2 n+1)}+\frac{1}{p q(n+1)}\right) \mathrm{e}^{-\frac{2 n+1}{n} t}
\end{aligned}
$$

hence the expansion in zonal functions by identification of the coefficients. Finally, for the structure spaces $\operatorname{USp}(n) / \mathrm{U}(n),\left(\phi^{(2,0, \ldots, 0)_{n}}\right)^{2}$ is equal to

$$
\begin{aligned}
& \frac{1}{2 n}\left(T\left[\left(\widetilde{g}_{11}\right)^{4}\right]+T\left[\left(\widetilde{g}_{11} \widetilde{g}_{22}\right)^{2}\right]+T\left[\left(\widetilde{g}_{12} \widetilde{g}_{21}\right)^{2}\right]\right) \\
& \quad+\frac{n-1}{n}\left(T\left[\left(\widetilde{g}_{13} \widetilde{g}_{24}\right)^{2}\right]+T\left[\left(\widetilde{g}_{11} \widetilde{g}_{33}\right)^{2}\right]+T\left[\left(\widetilde{g}_{13} \widetilde{g}_{31}\right)^{2}\right]\right)
\end{aligned}
$$

plus some remainder whose expectation under Brownian measures will be zero. Hence,

$$
\mathbb{E}\left[\left(\phi^{(2,0, \ldots, 0)_{n}}\right)^{2}\right]=\frac{1}{n(2 n+1)}+\frac{4(n-1)(n+1)}{3 n(2 n+1)} \mathrm{e}^{-\frac{2 n+1}{n} t}+\frac{n+1}{3 n} \mathrm{e}^{-\frac{2 n+4}{n} t},
$$

and $\frac{2 n+1}{n}$ is the exponent corresponding to the spherical representation of label $(2,2,0, \ldots, 0)_{n}$, whereas $\frac{2 n+4}{n}$ is the exponent corresponding to the spherical representation of label $(4,0, \ldots, 0)_{n}$.

\subsection{Proof of the Lower Bound on the Total Variation Distance}

The proof of the lower bound is now a simple application of Bienaymé-Chebyshev inequality. First, under the Haar measure, we have:

Proposition 27 If $E_{a}$ is the event $\{|\Omega| \geq a\}$, then the Haar measure of $E_{a}$ satisfies the inequality

$$
\eta_{X}\left(E_{a}\right) \leq \frac{1}{a^{2}}
$$

for every classical simple compact Lie group $X=K$ and every classical simple compact symmetric space $X=G / K$.

Proof The previous computations ensure that $\mathbb{E}_{\infty}\left[|\Omega|^{2}\right]=1$ in every case, so

$$
\eta_{X}[|\Omega| \geq a]=\eta_{X}\left[|\Omega|^{2} \geq a^{2}\right] \leq \frac{\mathbb{E}_{\infty}\left[|\Omega|^{2}\right]}{a^{2}}=\frac{1}{a^{2}} .
$$

Next, let us estimate $\mathbb{E}_{t}[\Omega]$ and $\operatorname{Var}_{t}[\Omega]$ for $t=\alpha(1-\varepsilon) \log n$. The exact values are listed in the table on the following page. We assume $\varepsilon<\frac{1}{4}$; indeed, Lemma 3 ensures that it is sufficient to control the total variation distance around the mixing time. We shall use a lot the inequality of convexity

$$
\exp (x) \leq 1+\frac{\mathrm{e}^{y}-1}{y} x \quad \forall x \in(0, y) .
$$


Lemma 28 Under the usual assumptions on $n$, for groups and spaces of structures (but not for Grassmannian varieties), $\operatorname{Var}_{t}[\Omega]$ is uniformly bounded for every $t=\alpha(1-$ $\varepsilon) \log n$ with $\varepsilon \in(0,1 / 4)$. Possible upper bounds are listed below:

$$
\begin{aligned}
& \mathrm{SU}(n), \operatorname{SU}(n) / \mathrm{SO}(n), \operatorname{SU}(2 n) / \mathrm{USp}(n): 1 ; \\
& \mathrm{SO}(2 n) / \mathrm{U}(n), \operatorname{USp}(n), \operatorname{USp}(n) / \mathrm{U}(n): 3 ; \\
& \mathrm{SO}(n): 8
\end{aligned}
$$

Proof We proceed case by case, and denote $\Delta_{t}(\lambda, \mu)=\mathrm{e}^{-\lambda t}-\mathrm{e}^{-\mu t}$. Notice that $\Delta_{t}(\lambda, \mu) \leq 0$ if $\lambda \geq \mu$. On the other hand, $\Delta_{t}(\lambda, \mu)$ is always smaller than 1 for $\lambda, \mu \geq 0$.

$-\operatorname{SO}(n)$ :

$$
\begin{aligned}
\operatorname{Var}_{t}[\Omega] & =\Delta_{t}\left(0, \frac{n-1}{n}\right)+\frac{n(n-1)}{2} \Delta_{t}\left(\frac{n-4}{n}, \frac{n-1}{n}\right)+\left(\frac{n(n+1)}{2}-1\right) \Delta_{t}\left(1, \frac{n-1}{n}\right) \\
& \leq 1+\frac{n(n-1)}{2} \Delta_{t}\left(\frac{n-4}{n}, \frac{n-1}{n}\right)=1+\frac{n(n-1)}{2} \mathrm{e}^{-\frac{n-1}{n} t}\left(\mathrm{e}^{\frac{3 t}{n}}-1\right) \\
& \leq 1+\frac{13}{2} n \log n \mathrm{e}^{-\frac{n-1}{n} t}
\end{aligned}
$$

\begin{tabular}{|c|c|c|}
\hline$K$ or $G / K$ & $\mathbb{E}_{t}[\Omega]$ & $\operatorname{Var}_{t}[\Omega]$ \\
\hline $\mathrm{SO}(n)$ & $n \mathrm{e}^{-\frac{n-1}{2 n} t}$ & $1+\frac{n(n-1)}{2} \mathrm{e}^{-\frac{n-4}{n} t}+\left(\frac{n(n+1)}{2}-1\right) \mathrm{e}^{-t}-n^{2} \mathrm{e}^{-\frac{n-1}{n} t}$ \\
\hline $\mathrm{SU}(n)$ & $n \mathrm{e}^{-\frac{n^{2}-1}{2 n^{2}} t}$ & $1+\left(n^{2}-1\right) \mathrm{e}^{-t}-n^{2} \mathrm{e}^{-\frac{n^{2}-1}{n^{2}} t}$ \\
\hline $\operatorname{USp}(n)$ & $2 n \mathrm{e}^{-\frac{2 n+1}{4 n} t}$ & $1+(2 n+1)(n-1) \mathrm{e}^{-t}+(2 n+1) n \mathrm{e}^{-\frac{n+1}{n} t}-4 n^{2} \mathrm{e}^{-\frac{2 n+1}{2 n} t}$ \\
\hline $\operatorname{Gr}(n, q, \mathbb{R})$ & $\sqrt{\frac{(n+2)(n-1)}{2}} \mathrm{e}^{-t}$ & $\begin{array}{c}1+\left(\frac{2 n^{2}}{p q}-8\right) \frac{(n-1)(n+2)}{(n-2)(n+4)} \mathrm{e}^{-t}+\frac{n^{2}}{3}\left(\frac{n+2}{n-2}-\frac{(n+2)(n-1)}{p q(n-2)}\right) \mathrm{e}^{-\frac{2 n-2}{n} t} \\
+\frac{n^{2}}{6}\left(\frac{n-1}{n+4}+\frac{2(n+2)(n-1)}{p q(n+4)}\right) \mathrm{e}^{-\frac{2 n+4}{n} t}-\frac{(n+2)(n-1)}{2} \mathrm{e}^{-2 t}\end{array}$ \\
\hline $\operatorname{Gr}(n, q, \mathbb{C})$ & $\sqrt{n^{2}-1} \mathrm{e}^{-t}$ & $\begin{array}{c}1+\left(\frac{2 n^{2}}{p q}-8\right) \frac{n^{2}-1}{n^{2}-4} \mathrm{e}^{-t}+\frac{n^{2}}{2}\left(\frac{n+1}{n-2}-\frac{n^{2}-1}{p q(n-2)}\right) \mathrm{e}^{-\frac{2 n-2}{n} t} \\
+\frac{n^{2}}{2}\left(\frac{n-1}{n+2}+\frac{n^{2}-1}{p q(n+2)}\right) \mathrm{e}^{-\frac{2 n+2}{n} t}-\left(n^{2}-1\right) \mathrm{e}^{-2 t}\end{array}$ \\
\hline $\operatorname{Gr}(n, q, \mathbb{H})$ & $\sqrt{(2 n+1)(n-1)} \mathrm{e}^{-t}$ & $\begin{aligned} 1+ & \left(\frac{n^{2}}{p q}-4\right) \frac{(n-1)(2 n+1)}{(n-2)(n+1)} \mathrm{e}^{-t}+\frac{n^{2}}{3}\left(\frac{2 n+1}{n-2}-\frac{(2 n+1)(n-1)}{p q(n-2)}\right) \mathrm{e}^{-\frac{2 n-2}{n} t} \\
& +\frac{n^{2}}{3}\left(\frac{4(n-1)}{(n+1)}+\frac{(2 n+1)(n-1)}{p q(n+1)}\right) \mathrm{e}^{-\frac{2 n+1}{n} t}-(2 n+1)(n-1) \mathrm{e}^{-2 t}\end{aligned}$ \\
\hline $\mathrm{SO}(2 n) / \mathrm{U}(n)$ & $\sqrt{n(2 n-1)} \mathrm{e}^{-\frac{n-1}{n} t}$ & $1+\frac{(n-1)(2 n-1)}{3} \mathrm{e}^{-\frac{2 n-4}{n} t}+\frac{4\left(n^{2}-1\right)}{3} \mathrm{e}^{-\frac{2 n-1}{n} t}-n(2 n-1) \mathrm{e}^{-\frac{2 n-2}{n} t}$ \\
\hline $\mathrm{SU}(n) / \mathrm{SO}(n)$ & $\sqrt{\frac{n(n+1)}{2}} \mathrm{e}^{-\frac{(n-1)(n+2)}{n^{2}} t}$ & $1+\frac{(n+2)(n-1)}{2} \mathrm{e}^{-\frac{2 n+2}{n} t}-\frac{n(n+1)}{2} \mathrm{e}^{-\frac{(n-1)(2 n+4)}{n^{2}} t}$ \\
\hline $\mathrm{SU}(2 n) / \mathrm{USp}(n)$ & $\sqrt{2 n^{2}-n} \mathrm{e}^{-\frac{(n-1)(2 n+1)}{2 n^{2}} t}$ & $1+\left(2 n^{2}-n-1\right) \mathrm{e}^{-\frac{2 n-1}{n} t}-\left(2 n^{2}-n\right) \mathrm{e}^{-\frac{(n-1)(2 n+1)}{n^{2}} t}$ \\
\hline$\underline{\mathrm{USp}}(n) / \mathrm{U}(n)$ & $\sqrt{n(2 n+1)} \mathrm{e}^{-\frac{n+1}{n} t}$ & $1+\frac{4(n-1)(n+1)}{3} \mathrm{e}^{-\frac{2 n+1}{n} t}+\frac{(2 n+1)(n+1)}{3} \mathrm{e}^{-\frac{2 n+4}{n} t}-n(2 n+1) \mathrm{e}^{-\frac{2 n+2}{n} t}$ \\
\hline
\end{tabular}

since $\frac{6 \log n}{n} \leq 1.382$ when $n \geq 10$, and $\frac{\mathrm{e}^{1.382}-1}{1.382} \leq \frac{13}{6}$. Then,

$$
\mathrm{e}^{-\frac{n-1}{n} t} \leq \mathrm{e}^{-\frac{3(n-1) \log n}{2 n}}=n^{-1} \mathrm{e}^{-\frac{(n-3) \log n}{2 n}} \leq \frac{14}{13}(n \log n)^{-1}
$$

for $n \geq 10$, so $\operatorname{Var}_{t}[\Omega] \leq 1+7=8$. 
- $\mathrm{SU}(n)$ :

$$
\operatorname{Var}_{t}[\Omega]=\Delta_{t}\left(0, \frac{n^{2}-1}{n^{2}}\right)+\left(n^{2}-1\right) \Delta_{t}\left(1, \frac{n^{2}-1}{n^{2}}\right) \leq 1
$$

- $\operatorname{USp}(n)$ :

$$
\begin{aligned}
\operatorname{Var}_{t}[\Omega]= & \Delta_{t}\left(0, \frac{2 n+1}{2 n}\right)+(2 n+1)(n-1) \Delta_{t}\left(1, \frac{2 n+1}{2 n}\right) \\
& +(2 n+1) n \Delta_{t}\left(\frac{2 n+2}{2 n}, \frac{2 n+1}{2 n}\right) \\
\leq & 1+(2 n+1)(n-1) \Delta_{t}\left(1, \frac{2 n+1}{2 n}\right) \leq 1+2 n^{2} \mathrm{e}^{-\frac{2 n+1}{2 n} t}\left(\mathrm{e}^{\frac{t}{2 n}}-1\right) \\
\leq & 1+\frac{5}{2} n \log n \mathrm{e}^{-\frac{2 n+1}{2 n} t}
\end{aligned}
$$

since $\frac{\log n}{n} \leq 0.367$ when $n \geq 3$, and $\frac{\mathrm{e}^{0.367}-1}{0.367} \leq \frac{5}{4}$. Then,

$$
\mathrm{e}^{-\frac{2 n+1}{2 n} t} \leq \mathrm{e}^{-\frac{3 \log n}{2}}=n^{-\frac{3}{2}} \leq \frac{4}{5}(n \log n)^{-1}
$$

for $n \geq 3$, so $\operatorname{Var}_{t}[\Omega] \leq 1+2=3$.

- $\mathrm{SO}(2 n) / \mathrm{U}(n)$ :

$$
\begin{aligned}
\operatorname{Var}_{t}[\Omega]= & \Delta_{t}\left(0, \frac{2 n-2}{n}\right)+\frac{(n-1)(2 n-1)}{3} \Delta_{t}\left(\frac{2 n-4}{n}, \frac{2 n-2}{n}\right) \\
& +\frac{4\left(n^{2}-1\right)}{3} \Delta_{t}\left(\frac{2 n-1}{n}, \frac{2 n-2}{n}\right) \\
\leq & 1+\frac{(n-1)(2 n-1)}{3} \Delta_{t}\left(\frac{2 n-4}{n}, \frac{2 n-2}{n}\right) \leq 1+\frac{2 n^{2}}{3} \mathrm{e}^{-\frac{2 n-2}{n} t}\left(\mathrm{e}^{\frac{2 t}{n}}-1\right) \\
\leq & 1+\frac{20}{9} n \log 2 n \mathrm{e}^{-\frac{2 n-2}{n} t}
\end{aligned}
$$

since $\frac{2 \log 2 n}{n} \leq 0.922$ when $2 n \geq 10$, and $\frac{\mathrm{e}^{0.922}-1}{0.922} \leq \frac{5}{3}$. Since

$$
\mathrm{e}^{-\frac{2 n-2}{n} t} \leq \mathrm{e}^{-\frac{3(n-1) \log 2 n}{2 n}}=\frac{1}{2} n^{-1} \mathrm{e}^{-\frac{(n-3) \log 2 n}{2 n}} \leq \frac{3}{4}(n \log 2 n)^{-1}
$$

for $2 n \geq 10$, one concludes that $\operatorname{Var}_{t}[\Omega] \leq 1+\frac{5}{3} \leq 3$.

- $\mathrm{SU}(n) / \mathrm{SO}(n)$ :

$\operatorname{Var}_{t}[\Omega]=\Delta_{t}\left(0, \frac{2(n-1)(n+2)}{n^{2}}\right)+\frac{(n+2)(n-1)}{2} \Delta_{t}\left(\frac{2(n+1)}{n}, \frac{2(n-1)(n+2)}{n^{2}}\right) \leq 1$.

- $\operatorname{SU}(2 n) / \mathrm{USp}(n)$ :

$\operatorname{Var}_{t}[\Omega]=\Delta_{t}\left(0, \frac{(n-1)(2 n+1)}{n^{2}}\right)+\left(2 n^{2}-n-1\right) \Delta_{t}\left(\frac{2 n-1}{n}, \frac{(n-1)(2 n+1)}{n^{2}}\right) \leq 1$. 
- $\operatorname{USp}(n) / \mathrm{U}(n)$ :

$$
\begin{aligned}
\operatorname{Var}_{t}[\Omega]= & \Delta_{t}\left(0, \frac{2 n+2}{n}\right)+\frac{4\left(n^{2}-1\right)}{3} \Delta_{t}\left(\frac{2 n+1}{n}, \frac{2 n+2}{n}\right) \\
& +\frac{2 n^{2}+3 n+1}{3} \Delta_{t}\left(\frac{2 n+4}{n}, \frac{2 n+2}{n}\right) \\
\leq & 1+\frac{4\left(n^{2}-1\right)}{3} \Delta_{t}\left(\frac{2 n+1}{n}, \frac{2 n+2}{n}\right) \leq 1+\frac{4 n^{2}}{3} \mathrm{e}^{-\frac{2 n+2}{n} t}\left(\mathrm{e}^{\frac{t}{n}}-1\right) \\
\leq & 1+\frac{5}{3} n \log n \mathrm{e}^{-\frac{2 n+2}{n} t}
\end{aligned}
$$

by using the same estimate on $\frac{\log n}{n}$ as in the case of USp $(n)$. Since

$$
\mathrm{e}^{-\frac{2 n+2}{n} t} \leq \mathrm{e}^{-\frac{3 \log n}{2}}=n^{-\frac{3}{2}} \leq \frac{4}{5}(n \log n)^{-1}
$$

for $n \geq 3$, one obtains $\operatorname{Var}_{t}[\Omega] \leq 1+\frac{4}{3} \leq 3$.

It is not possible to prove such uniform bounds for Grassmannians, because of the term $\mathrm{e}^{-t}$ that appears in the variance. We shall address this problem in Lemma 30.

Proposition 29 Denote $K_{X}$ the bound computed in the previous Lemma for the variance of the discriminating zonal function $\Omega$ associated to a space $X$. Then,

$$
d_{\mathrm{TV}}\left(\mu_{t}, \text { Haar }\right) \geq 1-\frac{4\left(K_{X}+1\right)}{\left(\mathbb{E}_{t}[\Omega]\right)^{2}} .
$$

Proof Assuming $a$ smaller than $m=\mathbb{E}_{t}[\Omega]$, if $|\Omega-m| \leq a$, then $|\Omega| \geq m-a$. Consequently,

$$
\mu_{t}[|\Omega| \geq m-a] \geq 1-\mathbb{P}[|\Omega-m|>a] \geq 1-\frac{\operatorname{Var}_{t}[\Omega]}{a^{2}}=1-\frac{K_{X}}{a^{2}} .
$$

Next, take $a=\frac{m}{2}$. The combination of Lemma 27 and of the previous inequality yields

$$
d_{\mathrm{TV}}\left(\mu_{t}, \text { Haar }\right) \geq \mu_{t}\left(E_{a}\right)-\eta_{X}\left(E_{a}\right) \geq 1-\frac{K_{X}+1}{a^{2}}=1-\frac{4\left(K_{X}+1\right)}{m^{2}} .
$$

Since $m^{2}$ behaves as $n^{2 \varepsilon}$, this essentially ends the proof of the lower bounds in the case of compact Lie groups and compact spaces of structures. More precisely:

- $\quad \mathrm{SO}(n): m^{2} \geq n^{2 \varepsilon}$ so the constant $c$ in our main Theorem 6 is $4(8+1)=36$.

- $\mathrm{SU}(n)$ : again, $m^{2} \geq n^{2 \varepsilon}$, so the constant is $4(1+1)=8$.

- $\operatorname{USp}(n)$ : here, $m^{2} \geq 4 n^{2 \varepsilon} \mathrm{e}^{-\frac{\log n}{2 n}} \geq \frac{16}{5} n^{2 \varepsilon}$ for $n \geq 3$, so the constant is $\frac{5}{16} 4(3+1)=5$.

- $\mathrm{SO}(2 n) / \mathrm{U}(n): m^{2} \geq \frac{2 n-1}{4 n}(2 n)^{2 \varepsilon} \geq \frac{9}{20}(2 n)^{2 \varepsilon}$ for $2 n \geq 10$, whence a constant $\frac{9}{20} 4(3+1)=\frac{36}{5} \leq 8$.

- $\mathrm{SU}(n) / \mathrm{SO}(n): m^{2} \geq \frac{n^{2 \varepsilon}}{2} \mathrm{e}^{-\frac{2(n-2) \log n}{n^{2}}} \geq \frac{n^{2 \varepsilon}}{3}$ for $n \geq 2$, so a possible constant is $3 \times$ $4(1+1)=24$. 
- $\operatorname{SU}(2 n) / \mathrm{USp}(n): m^{2} \geq \frac{2 n-1}{4 n}(2 n)^{2 \varepsilon} \geq \frac{3}{8}(2 n)^{2 \varepsilon}$, and a possible constant is $\frac{8}{3} 4(1+$ 1) $=\frac{64}{3} \leq 22$.

- $\operatorname{USp}(n) / \mathrm{U}(n): m^{2} \geq 2 n^{2 \varepsilon} \mathrm{e}^{-\frac{2 \log n}{n}} \geq \frac{16}{17} n^{2 \varepsilon}$ for $n \geq 3$, whence a constant $\frac{17}{16} 4(3+$ 1) $=17$.

Unfortunately, for Grassmannian varieties, the variance of $\Omega$ at time $t=(1-$ $\varepsilon) \log n$ can only be bounded by a constant times $n^{\varepsilon}$. However, since the mean of $\Omega$ is also of order $n^{\varepsilon}$, this will still ensure that the discriminating zonal spherical function has not at all the same behavior under Haar measure and under Brownian measures before cut-off time. The only downside is the loss of a factor $n^{\varepsilon}$ in the estimate of the total variation distance.

Lemma 30 Under the usual assumptions on n, for Grassmannian varieties,

$$
\frac{\operatorname{Var}_{t}[\Omega]}{n^{\varepsilon}} \leq \begin{cases}3 & \text { if } \mathbb{k}=\mathbb{R}, \\ 5 & \text { if } \mathbb{k}=\mathbb{C} \text { or } \mathbb{H},\end{cases}
$$

for every $t=\alpha(1-\varepsilon) \log n$ with $\varepsilon \in(0,1 / 4)$.

Proof The quantity $\frac{1}{p q}$ is bounded by

$$
\frac{4}{n^{2}} \leq \frac{1}{p q} \leq \frac{1}{n-1},
$$

the extremal values corresponding to $p=q=\frac{n}{2}$ and to $p=n-1$ or $q=n-1$. In particular, in the expansions hereafter, all the coefficients that precede differences of exponentials $\Delta_{t}(\lambda, \mu)$ are positive. Now, we proceed case by case:

$-\operatorname{Gr}(n, q, \mathbb{R})$ :

$$
\begin{aligned}
\operatorname{Var}_{t}[\Omega]= & \Delta_{t}(0,2)+\left(\frac{2 n^{2}}{p q}-8\right) \frac{(n-1)(n+2)}{(n-2)(n+4)} \Delta_{t}(1,2) \\
& +\frac{n^{2}}{3}\left(\frac{n+2}{n-2}-\frac{(n+2)(n-1)}{p q(n-2)}\right) \Delta_{t}\left(\frac{2 n-2}{n}, 2\right) \\
& +\frac{n^{2}}{6}\left(\frac{n-1}{n+4}+\frac{2(n+2)(n-1)}{p q(n+4)}\right) \Delta_{t}\left(\frac{2 n+4}{n}, 2\right) \\
\leq & 1+2 n \Delta_{t}(1,2)+\frac{n^{2}}{3} \Delta_{t}\left(\frac{2 n-2}{n}, 2\right) .
\end{aligned}
$$

For the difference $\Delta_{t}(1,2)$, one cannot obtain a better bound than $\mathrm{e}^{-t}=n^{\varepsilon-1}$. The second difference $\Delta_{t}\left(\frac{2 n-2}{n}, 2\right)$ is bounded from above by

$$
\mathrm{e}^{-2 t}\left(\mathrm{e}^{\frac{2 t}{n}}-1\right) \leq n^{-\frac{3}{2}} \frac{8 \log n}{3 n} \leq 2 n^{-2}
$$

by using similar arguments as in the proof of Lemma 28, and the inequality $n \geq$ 10. So,

$$
\operatorname{Var}_{t}[\Omega] \leq 1+\frac{2}{3}+2 n^{\varepsilon} \leq 3 n^{\varepsilon}
$$


- $\operatorname{Gr}(n, q, \mathbb{C})$ :

$$
\begin{aligned}
\operatorname{Var}_{t}[\Omega]= & \Delta_{t}(0,2)+\left(\frac{2 n^{2}}{p q}-8\right) \frac{n^{2}-1}{n^{2}-4} \Delta_{t}(1,2) \\
& +\frac{n^{2}}{2}\left(\frac{n+1}{n-2}-\frac{n^{2}-1}{p q(n-2)}\right) \Delta_{t}\left(\frac{2 n-2}{n}, 2\right) \\
& +\frac{n^{2}}{2}\left(\frac{n-1}{n+2}+\frac{n^{2}-1}{p q(n+2)}\right) \Delta_{t}\left(\frac{2 n+2}{2}, 2\right) \\
\leq & +2 n \Delta_{t}(1,2)+\frac{n^{2}}{2} \Delta_{t}\left(\frac{2 n-2}{n}, 2\right) .
\end{aligned}
$$

The second difference is controlled exactly as in the case of real Grassmannians, but under the constraint $n \geq 2$ :

$$
\Delta_{t}\left(\frac{2 n-2}{n}, 2\right) \leq \mathrm{e}^{-2 t}\left(\mathrm{e}^{\frac{2 t}{n}}-1\right) \leq n^{-\frac{3}{2}} \frac{3 \log n}{n} \leq \frac{9}{4} n^{-2} .
$$

Hence, $\operatorname{Var}_{t}[\Omega] \leq 1+\frac{9}{8}+2 n^{\varepsilon} \leq 5 n^{\varepsilon}$.

- $\operatorname{Gr}(n, q, \mathbb{H})$ :

$$
\begin{aligned}
\operatorname{Var}_{t}[\Omega]= & \Delta_{t}(0,2)+\frac{n^{2}}{3}\left(\frac{2 n+1}{n-2}-\frac{(2 n+1)(n-1)}{p q(n-2)}\right) \Delta_{t}\left(\frac{2 n-2}{n}, 2\right) \\
& +\left(\frac{n^{2}}{p q}-4\right) \frac{(n-1)(2 n+1)}{(n-2)(n+1)} \Delta_{t}(1,2) \\
& +\frac{n^{2}}{3}\left(\frac{4(n-1)}{(n+1)}+\frac{(2 n+1)(n-1)}{p q(n+1)}\right) \Delta_{t}\left(\frac{2 n+1}{n}, 2\right) \\
\leq & 1+2 n \Delta_{t}(1,2)+\frac{2 n^{2}}{3} \Delta_{t}\left(\frac{2 n-2}{n}, 2\right) \leq 1+2 n^{\varepsilon}+\frac{3}{2} \leq 5 n^{\varepsilon} .
\end{aligned}
$$

Now, Proposition 29 still holds, but with $K_{X}$ varying with $n$ and equal to $3 n^{\varepsilon}$ or $5 n^{\varepsilon}$ according to the field $\mathbb{k}=\mathbb{R}, \mathbb{C}$ or $\mathbb{H}$. Thus:

Proposition 31 For Grassmannian varieties $\operatorname{Gr}(n, q$, $\mathbb{k})$, if $t=(1-\varepsilon) \log n$ with $\varepsilon \in$ $(0,1 / 4)$, then

$$
d_{\mathrm{TV}}\left(\mu_{t}, \text { Haar }\right) \geq 1-\frac{L n^{\varepsilon}}{m^{2}} \quad \text { with } L= \begin{cases}16 & \text { if } \mathbb{k}=\mathbb{R} \\ 24 & \text { if } \mathbb{k}=\mathbb{C} \text { or } \mathbb{H} .\end{cases}
$$

Finally, the deduction of the constants in Theorem 6 goes as follows:

- $\operatorname{Gr}(n, q, \mathbb{R}): m \geq \frac{n^{2 \varepsilon}}{2}$, so the constant can be taken equal to $2 \times 16=32$.

- $\operatorname{Gr}(n, q, \mathbb{C}): m \geq \frac{n^{2}-1}{n^{2}} n^{2 \varepsilon} \geq \frac{3}{4} n^{2 \varepsilon}$, so a possible constant is again $\frac{4}{3} 24=32$.

- $\operatorname{Gr}(n, q, \mathbb{H}): m \geq \frac{2 n^{2}-n-1}{2} n^{2 \varepsilon} \geq \frac{3}{2} n^{2 \varepsilon}$ for $n \geq 3$, whence a constant $\frac{2}{3} 24=16$.

These computations end the proof of the cut-off phenomenon. 


\section{Appendices (Technical Computations)}

\subsection{Proof of the Upper Bound for Odd Special Orthogonal Groups}

With the same scheme of growth of partitions as for compact symplectic groups, one has the following bounds:

- $\quad \eta_{1, n}$ : it is given by the exact formula $\left(\begin{array}{c}2 n+1 \\ n+1\end{array}\right) \mathrm{e}^{-\frac{n(n+1)}{2 n+1} \log (2 n+1)}$, which is indeed smaller than 1 for $n \geq 5$.

- $\eta_{k \geq 2, n}$ : the comparison techniques between sums and integrals give

$$
\begin{aligned}
\log \eta_{k, n} \leq & -\frac{n(2 k-1+n)}{2 n+1} \log (2 n+1)+\frac{2}{2 k-1}+\frac{1}{k}+\log (k+n-2)-\log k \\
& +(2 k+2 n-2) \log (2 k+2 n-2)+(2 k-2) \log (2 k-2) \\
& -2(2 k+n-2) \log (2 k+n-2) .
\end{aligned}
$$

This bound is decreasing in $k$, whence smaller than its value when $k=2$, which is negative for every value of $n \geq 5$.

- $\quad \eta_{k, l \in \llbracket 3, n-1 \rrbracket}$ : as before, $\rho_{k, l}$ splits into $\rho_{k, l,(1)}$ and $\rho_{k, l,(2)}$ :

$$
\begin{aligned}
\rho_{k, l}= & \prod_{j=l+1}^{n} \frac{k+j-1+\lambda_{l+1}-\lambda_{j}}{k+j-l-1+\lambda_{l+1}-\lambda_{j}} \frac{k+\lambda_{l+1}+\lambda_{j}+2 n-j}{k+\lambda_{l+1}+\lambda_{j}+2 n-j-l} \\
& \times \prod_{1 \leq i \leq j \leq l} \frac{2 k+2 \lambda_{l+1}+2 n+1-i-j}{2 k+2 \lambda_{l+1}+2 n-1-i-j} .
\end{aligned}
$$

The bound on $\log \widetilde{\eta}_{k, l}$, the sum of $\log \rho_{k, l,(2)}$ and of the variation of $-\frac{t_{n, 0}}{2} B_{n}(\lambda)$, is

$$
\begin{aligned}
\log \widetilde{\eta}_{k, l} \leq & -\frac{l\left(2 k^{\prime}-1+2 n-l\right)}{2 n+1} \log (2 n+1)+\frac{1}{k^{\prime}+n-l-1} \\
& +\log \left(k^{\prime}+n-2\right)-\log \left(k^{\prime}+n-l-1\right) \\
& +\left(2 k^{\prime}+2 n-2\right) \log \left(2 k^{\prime}+2 n-2\right) \\
& +\left(2 k^{\prime}+2 n-2 l-2\right) \log \left(2 k^{\prime}+2 n-2 l-2\right) \\
& -2\left(2 k^{\prime}+2 n-l-2\right) \log \left(2 k^{\prime}+2 n-l-2\right) \\
\leq & -\frac{l(2 v+2 n+1-l)}{2 n+1} \log (2 n+1)+\frac{1}{v+n-l} \\
& +\log (v+n-1)-\log (v+n-l)+(2 v+2 n) \log (2 v+2 n) \\
& +(2 v+2 n-2 l) \log (2 v+2 n-2 l) \\
& -2(2 v+2 n-l) \log (2 v+2 n-l)
\end{aligned}
$$


with $k^{\prime}=k+\lambda_{l+1}=k+v$. On the other hand, in the product $\rho_{k, l,(1)}$, each term of index $j$ writes as

$$
\begin{aligned}
\frac{\left(k^{\prime}+n-1 / 2\right)^{2}-\left(\lambda_{j}+n+1 / 2-j\right)^{2}}{\left(k^{\prime}+n-1 / 2-l\right)^{2}-\left(\lambda_{j}+n+1 / 2-j\right)^{2}} & \leq \frac{\left(k^{\prime}+n-1 / 2\right)^{2}-\left(\lambda_{l+1}+n+1 / 2-j\right)^{2}}{\left(k^{\prime}+n-1 / 2-l\right)^{2}-\left(\lambda_{l+1}+n+1 / 2-j\right)^{2}} \\
& \leq \frac{k+j-1}{k+j-l-1} \frac{k^{\prime \prime}+2 n-j}{k^{\prime \prime}+2 n-j-l},
\end{aligned}
$$

so the quantity $\rho_{k, l,(1)}$ is bounded by

$$
\begin{aligned}
& \frac{(k+n-1) !}{(k+l-1) !} \frac{(k-1) !}{(k+n-l-1) !} \frac{\left(k^{\prime \prime}+2 n-l-1\right) !}{\left(k^{\prime \prime}+n-1\right) !} \frac{\left(k^{\prime \prime}+n-l-1\right) !}{\left(k^{\prime \prime}+2 n-2 l-1\right) !} \\
& \quad \leq \frac{n !(2 v+2 n-l) !(2 v+n-l) !}{l !(n-l) !(2 v+n) !(2 v+2 n-2 l) !} .
\end{aligned}
$$

Again, Stirling approximation leads to

$$
\begin{aligned}
\log \rho_{k, l,(1)} \leq & (2 v+2 n-l) \log (2 v+2 n-l)+(2 v+n-l) \log (2 v+n-l) \\
& -(2 v+n) \log (2 v+n)-(2 v+2 n-2 l) \log (2 v+2 n-2 l) \\
& +n \log n-l \log l-(n-l) \log (n-l)+\frac{1}{2 n-2},
\end{aligned}
$$

and therefore

$$
\begin{aligned}
\log \eta_{k, l} \leq & -\frac{l(2 v+2 n+1-l)}{2 n+1} \log (2 n+1)+\frac{1}{v+n-l}+\log (v+n-1)-\log (v+n-l) \\
& +(2 v+2 n) \log (2 v+2 n)+(2 v+n-l) \log (2 v+n-l) \\
& -(2 v+n) \log (2 v+n)-(2 v+2 n-l) \log (2 v+2 n-l) \\
& +n \log n-l \log l-(n-l) \log (n-l)+\frac{1}{2 n-2} \\
\leq & -\frac{l(2 n+1-l)}{2 n+1} \log (2 n+1)+\frac{1}{n-l}+\log (n-1)-\log (n-l) \\
& +n \log n-l \log l-(n-l) \log (n-l)+\frac{1}{2 n-2} .
\end{aligned}
$$

The last bound is decreasing in $l$, so it suffices to look at the case $l=3$; then the bound is decreasing in $n$, so it suffices to check that the bound is negative when $n=5$, which is just a computation. We conclude that $\log \eta_{k, l} \leq 0$ for any $k$ and any $l \in \llbracket 3, n-1 \rrbracket$. 
- $\quad \eta_{k, 1}$ : a bound on $\rho_{k, 1}$ is $\frac{k+n-2}{k} \frac{2 k+2 n-1}{2 k+2 n-3}$, so

$$
\begin{aligned}
\eta_{k, 1} & \leq \frac{k+n-2}{k} \frac{2 k+2 n-1}{2 k+2 n-3} \mathrm{e}^{-\frac{2 k+2 n-2}{2 n+1} \log (2 n+1)} \leq \frac{(n-1)(2 n+1)}{2 n-1} \mathrm{e}^{-\frac{2 n}{2 n+1} \log (2 n+1)} \\
& \leq \frac{n-1}{2 n-1} \mathrm{e}^{\frac{\log (2 n+1)}{2 n+1}} \leq \frac{1}{2} \mathrm{e}^{\frac{\log 11}{11}} \leq 1
\end{aligned}
$$

- $\quad \eta_{k, 2}$ : a bound on $\rho_{k, 2}$ is $\frac{k+2 n-4}{k} \frac{k+2 n-3}{k+1} \frac{2 k+2 n-1}{2 k+2 n-5} \frac{k+n-1}{k+n-2}$, so

$$
\eta_{k, 2} \leq \frac{k+2 n-4}{k} \frac{k+2 n-3}{k+1} \frac{2 k+2 n-1}{2 k+2 n-5} \frac{k+n-1}{k+n-2} \mathrm{e}^{-\frac{4 k+4 n-6}{2 n+1} \log (2 n+1)} \leq \frac{n}{2 n+1} \mathrm{e}^{\frac{4 \log (2 n+1)}{2 n+1}} .
$$

The last bound is bigger than 1 only when $n=5$ or 6 . The maximal value is obtained for $n=5$, and is smaller than $1.09 \leq \frac{11}{10}$. Moreover, if $k \geq 2$, then one has a much better bound, that is smaller than 1 even when $n=5$ or 6 .

Putting all together, one sees that at most one quotient $\eta_{k, l}$ may be bigger than 1 (and actually only when $n=5$ or 6 ). Thus, we have proved Proposition 17.

\subsection{Proof of the Upper Bound for Even Special Orthogonal Groups}

We analyze as before the various quotients $\rho_{k, l}$ and $\eta_{k, l}$ corresponding to the growth of partition described by Eq. 3.2:

- $\quad \eta_{k, n}:$ the general formula is

$$
\eta_{k, n}=\left(\prod_{i=1}^{n-1} \frac{2 k+2 n-2 i-1}{2 k+n-i-1} \frac{2 k+2 n-2 i-2}{2 k+n-i-2}\right) \mathrm{e}^{-\frac{2 k+n-2}{2} \log (2 n)}
$$

which is decreasing in $k$ and reduces to $\left(\begin{array}{c}2 n-1 \\ n\end{array}\right) \mathrm{e}^{-\frac{n \log (2 n)}{2}}$ when $k=1$. This latter bound is always smaller than 1 .

- $\quad \eta_{k, l \in \llbracket 2, n-1 \rrbracket}$ : the quotient of dimensions $\rho_{k, l}=\rho_{k, l,(1)} \rho_{k, l,(2)}$ is equal to

$$
\begin{aligned}
& \prod_{j=l+1}^{n} \frac{k+j-1+\lambda_{l+1}-\lambda_{j}}{k+j-l-1+\lambda_{l+1}-\lambda_{j}} \frac{k+\lambda_{l+1}+\lambda_{j}+2 n-1-j}{k+\lambda_{l+1}+\lambda_{j}+2 n-1-j-l} \\
& \quad \times \prod_{1 \leq i<j \leq l} \frac{2 k+2 \lambda_{l+1}+2 n-i-j}{2 k+2 \lambda_{l+1}+2 n-2-i-j} .
\end{aligned}
$$

The main difference with the previous computations is that $\rho_{k, l,(2)}$ is a product over distinct indices $i<j$, so we will not have to worry about diagonal terms 
in the corresponding sum (see the argument at the beginning of Section 3.2.1). Hence, with the same notations as before,

$$
\begin{aligned}
\log \widetilde{\eta}_{k, l} \leq & -\frac{l\left(2 k^{\prime}-2+2 n-l\right)}{2 n} \log (2 n)+\left(2 k^{\prime}+2 n-3\right) \log \left(2 k^{\prime}+2 n-3\right) \\
& +\left(2 k^{\prime}+2 n-2 l-3\right) \log \left(2 k^{\prime}+2 n-2 l-3\right) \\
& -2\left(2 k^{\prime}+2 n-l-3\right) \log \left(2 k^{\prime}+2 n-l-3\right) \\
\leq & -\frac{l(2 v+2 n-l)}{2 n} \log (2 n)+(2 v+2 n-1) \log (2 v+2 n-1) \\
& +(2 v+2 n-2 l-1) \log (2 n-2 l-1) \\
& -2(2 v+2 n-l-1) \log (2 v+2 n-l-1) ; \\
\log \rho_{k, l,(1)} & (2 v+2 n-l-1) \log (2 v+2 n-l-1) \\
& +(2 v+n-l-1) \log (2 v+n-l-1) \\
& -(2 v+n-1) \log (2 v+n-1) \\
& -(2 v+2 n-2 l-1) \log (2 v+2 n-2 l-1) \\
& +n \log n-l \log l-(n-l) \log (n-l)+\frac{1}{2 n-2} .
\end{aligned}
$$

Adding together these bounds, using the concavity of $x \log x$ and then the decreasing character with respect to $v$ gives

$$
\begin{aligned}
\log \eta_{k, l}= & \log \tilde{\eta}_{k, l}+\log \rho_{k, l,(1)} \leq-\frac{l(2 n-l)}{2 n} \log (2 n) \\
& +n \log n-l \log l-(n-l) \log (n-l)+\frac{1}{2 n-2},
\end{aligned}
$$

which is decreasing in $l \geq 2$. Then,

$$
-\frac{2 n-2}{n} \log (2 n)+n \log (n)-2 \log 2-(n-2) \log (n-2)+\frac{1}{2 n-2}
$$

is decreasing in $n$, and one can check that it is negative when $n=5$. So, $\eta_{k, l} \leq 1$ for any $k$ and any $l \in \llbracket 2, n-1 \rrbracket$.

- $\quad \eta_{k, 1}$ : one has $\rho_{k, 1} \leq \frac{k+2 n-3}{k} \frac{k+n-1}{k+n-2}$, and therefore

$$
\eta_{k, 1} \leq \frac{k+2 n-3}{k} \frac{k+n-1}{k+n-2} \mathrm{e}^{-\frac{2 n+2 k-3}{2 n} \log (2 n)} .
$$

Suppose $k \geq 2$; then the right-hand side is smaller than $\frac{2 n-1}{2 n} \frac{n+1}{2 n}$, so $\eta_{k, 1} \leq 1$. On the other hand, for $k=1$, which happens only once,

$$
\eta_{1,1} \leq \mathrm{e}^{\frac{\log (2 n)}{2 n}} \leq \mathrm{e}^{\frac{\log 10}{10}} \leq \frac{4}{3}
$$

This proves Proposition 18. 


\subsection{Proof of the Upper Bound for Complex Grassmannians}

For a partition of size $p=\left\lfloor\frac{n}{2}\right\rfloor$, one has $B_{n}(\lambda)=\frac{2}{n} \sum_{i=1}^{p} \lambda_{i}^{2}+(n+1-2 i) \lambda_{i}$ and either

$$
A_{n}(\lambda)=\left(\prod_{i=1}^{p} \prod_{j=1}^{p} \frac{\lambda_{i}+\lambda_{j}+n+1-i-j}{n+1-i-j}\right)\left(\prod_{1 \leq i<j \leq p} \frac{\lambda_{i}-\lambda_{j}+j-i}{j-i}\right)^{2}
$$

if $n=2 p$, or

$$
A_{n}(\lambda)=\left(\prod_{i=1}^{p+1} \prod_{j=1}^{p+1} \frac{\lambda_{i}+\lambda_{j}+n+1-i-j}{n+1-i-j}\right)\left(\prod_{1 \leq i<j \leq p} \frac{\lambda_{i}-\lambda_{j}+j-i}{j-i}\right)^{2}
$$

when $n=2 p+1$. Let us give the details when $n=2 p$. Again, one looks at $\rho_{k, l}=$ $A_{n}(\lambda) / A_{n}(\mu)$ and $\eta_{k, l}=\rho_{k, l} \mathrm{e}^{-\log n\left(B_{n}(\lambda)-B_{n}(\mu)\right)}$, with $\mu$ and $\lambda$ equal to

$$
\begin{aligned}
& \left(\lambda_{l+1}+k-1, \ldots, \lambda_{l+1}+k-1, \lambda_{l+1}, \ldots, \lambda_{p}\right)_{p} \quad \text { and } \\
& \left(\lambda_{l+1}+k, \ldots, \lambda_{l+1}+k, \lambda_{l+1}, \ldots, \lambda_{p}\right)_{p} .
\end{aligned}
$$

The quotient of dimensions is

$$
\begin{aligned}
\rho_{k, l}= & \left(\prod_{j=1}^{l} \frac{\left(2 k^{\prime}+n-j\right)\left(2 k^{\prime}+n-j-1\right)}{\left(2 k^{\prime}+n-j-l\right)\left(2 k^{\prime}+n-j-l-1\right)}\right) \\
& \times\left(\prod_{j=l+1}^{p} \frac{\left(k^{\prime}-\lambda_{j}+j-1\right)\left(k^{\prime}+\lambda_{j}+n-j\right)}{\left(k^{\prime}-\lambda_{j}+j-l-1\right)\left(k^{\prime}+\lambda_{j}+n-j-l\right)}\right)^{2},
\end{aligned}
$$

and a lower bound is then obtained by the usual replacement $\lambda_{l+1}=\lambda_{j}=0$ and then $k=1$ :

$$
\rho_{k, l} \leq \frac{n-2 l+1}{n+1}\left(\begin{array}{c}
n+1 \\
l
\end{array}\right)^{2} \quad ; \quad \eta_{k, l} \leq \frac{n-2 l+1}{n+1}\left(\begin{array}{c}
n+1 \\
l
\end{array}\right)^{2} \mathrm{e}^{-\frac{2 l(n+1-l)}{n} \log n}
$$

The last quantity is decreasing in $l$, as the quotient of two consecutive terms of parameters $n, l$ and $n, l+1$ is smaller than

$$
\left(\frac{n+1-l}{l+1} \mathrm{e}^{-\frac{n-2 l}{n} \log n}\right)^{2} \leq 1
$$

So,

$$
\eta_{k, l} \leq \frac{n-1}{n+1}(n+1)^{2} \mathrm{e}^{-2 \log n}=\frac{n^{2}-1}{n^{2}} \leq 1
$$

and $A_{n}(\lambda) \mathrm{e}^{-\log n B_{n}(\lambda)}$ is smaller than 1 for any partition (we leave to the reader the verification of the other case $n=2 p+1$, which is very similar). 


\subsection{Expansion of Elementary 4-Tensors for Unitary Groups}

For the eigenvectors associated to the value $2 n-2$, we shall write

$$
\begin{aligned}
S(i, j, k, l)= & (e[i, j]-e[j, i])^{\otimes 2}-(e[j, k]-e[k, j])^{\otimes 2} \\
& +(e[k, l]-e[l, k])^{\otimes 2}-(e[l, i]-e[i, l])^{\otimes 2} .
\end{aligned}
$$

The elementary tensor $e_{i}^{\otimes 4}$ is equal to

$$
\begin{aligned}
& \frac{1}{n(n+1)} \sum_{k, l=1}^{n} e[k, l, k, l]+e[k, l, l, k]+\frac{1}{n(n+2)} \sum_{k \neq i} \sum_{l=1}^{n}\left(\begin{array}{c}
(e[i, l, i, l]-e[k, l, k, l])+(e[l, i, l, i]-e[l, k, l, k]) \\
+(e[i, l, l, i]-e[k, l, l, k])+(e[l, i, i, l]-e[l, k, k, l])
\end{array}\right) \\
& \quad+\frac{1}{n+2} \sum_{k \neq i} e_{i}^{\otimes 4}+e_{k}^{\otimes 4}-(e[i, k]+e[k, i])^{\otimes 2} \\
& \quad-\frac{1}{(n+1)(n+2)} \sum_{k<l} e_{k}^{\otimes 4}+e_{l}^{\otimes 4}-(e[k, l]+e[l, k])^{\otimes 2}
\end{aligned}
$$

with the two first terms respectively in $V_{2 n^{2}-2}$ and $V_{n^{2}-2}$, and the second line in $V_{-2 n-2}$. Similarly, $e_{i} \otimes e_{j} \otimes e_{i} \otimes e_{j}$ is equal to

$$
\begin{aligned}
& \frac{1}{(n-1)(n+1)} \sum_{k=1}^{n} \sum_{l=1}^{n} e[k, l, k, l]-\frac{1}{n(n-1)(n+1)} \sum_{k=1}^{n} \sum_{l=1}^{n} e[k, l, l, k] \\
& +\frac{1}{n(n+2)}\left(\sum_{l=1}^{n}\left(\begin{array}{c}
e[i, l, i, l]-e[j, l, j, l] \\
+e[l, j, l, j]-e[l, i, l, i]
\end{array}\right)\right)+\frac{1}{(n-2)(n+2)} \sum_{k \neq i, j}\left(\sum_{l=1}^{n}\left(\begin{array}{c}
e[i, l, i, l]-e[k, l, k, l] \\
+e_{l}[, j, j, l]-e[l, k, l, k]
\end{array}\right)\right) \\
& -\frac{1}{n(n-2)(n+2)} \sum_{k \neq i, j}\left(\sum_{l=1}^{n}\left(\begin{array}{c}
e[i, l, l, i]+e[j, l, l, j]-2 e[k, l, l, k] \\
+e[l, i, i, l]+e[l, j, j, l]-2 e[l, k, k, l]
\end{array}\right)\right) \\
& +\frac{1}{4(n-1)(n-2)} \sum_{\left(\begin{array}{c}
k<l) \neq i, j \\
l
\end{array}\right.} 2 S(i, j, k, l)-S(i, k, j, l)+\frac{1}{2 n} \sum_{k \neq i, j}\left(\begin{array}{c}
e[i, j, i, j]+e[j, k, j, k]+e[k, i, k, i] \\
-e[j, j, j]-e[k, j, k, j]-e[i, k, i, k]
\end{array}\right) \\
& +\frac{1}{4(n+2)}\left(\sum_{k \neq i} e_{i}^{\otimes 4}+e_{k}^{\otimes 4}-(e[i, k]+e[k, i])^{\otimes 2}+\sum_{k \neq j} e_{j}^{\otimes 4}+e_{k}^{\otimes 4}-(e[j, k]+e[k, j])^{\otimes 2}\right) \\
& -\frac{1}{4}\left(e_{i}^{\otimes 4}+e_{j}^{\otimes 4}-(e[i, j]+e[j, i])^{\otimes 2}\right) \\
& -\frac{1}{2(n+1)(n+2)}\left(\sum_{k<l} e_{k}^{\otimes 4}+e_{l}^{\otimes 4}-(e[k, l]+e[l, k])^{\otimes 2}\right)
\end{aligned}
$$

with the parts of this expansion respectively in $V_{2 n^{2}-2}, V_{n^{2}-2}, V_{2 n-2}, V_{-2}$ and $V_{-2 n-2}$.

\subsection{Expansion of Elementary 4-Tensors for Compact Symplectic Groups}

It is a little more tedious than before to find a complete list of "simple" eigenvectors of $M_{n, 4}$ (or at least a sufficient list to expand simple tensors). The list of possible 
eigenvalues of $M_{n, 4}$ is $\{2 n+1, n+1, n, 3,1,0,-1,-3\}$, and on the other hand, one can easily identify a basis of $V_{2 n+1}$ : it consists in the three vectors

$$
\begin{aligned}
& v_{2 n+1,1}=\sum_{i, j=1}^{n}\left(\begin{array}{c}
e[2 i-1,2 i, 2 j-1,2 j]+e[2 i, 2 i-1,2 j, 2 j-1] \\
-e[2 i, 2 i-1,2 j-1,2 j]-e[2 i-1,2 i, 2 j, 2 j-1]
\end{array}\right) ; \\
& v_{2 n+1,2}=\sum_{i, j=1}^{n}\left(\begin{array}{c}
e[2 i-1,2 j-1,2 i, 2 j]+e[2 i, 2 j, 2 i-1,2 j-1] \\
-e[2 i, 2 j-1,2 i-1,2 j]-e[2 i-1,2 j, 2 i, 2 j-1]
\end{array}\right) ; \\
& v_{2 n+1,3}=\sum_{i, j=1}^{n}\left(\begin{array}{c}
e[2 i-1,2 j-1,2 j, 2 i]+e[2 i, 2 j, 2 j-1,2 i-1] \\
-e[2 i, 2 j-1,2 j, 2 i-1]-e[2 i-1,2 j, 2 j-1,2 i]
\end{array}\right) .
\end{aligned}
$$

But then, it becomes really difficult to describe the other eigenspaces. However, one can still find the eigenvector expansion of simple tensors such as $e_{i}^{\otimes 4}, e_{i}^{\otimes 2} e_{j}^{\otimes 2}$, or $e[i, j, k, l]$; hence, in the following, we just give these expansions (again it is easy to check that each part of an expansion is indeed an eigenvector). The tensor $e[i, i, i, i]$ is an eigenvector in $V_{-3}$, and on the other hand, $e[2 i-1,2 i-1,2 i, 2 i]$ decomposes into the eigenvectors

$$
\begin{aligned}
& \frac{1}{2 n(2 n+1)}\left(v_{2 n+1,2}+v_{2 n+1,3}\right) \\
& +\frac{n-2}{4 n(n+1)} \sum_{\sigma \in S} \sum_{j \neq i}\left(\begin{array}{c}
e[2 i-1,2 j-1,2 i, 2 j]+e[2 i, 2 j, 2 i-1,2 j-1] \\
-e[2 i-1,2 j, 2 i, 2 j-1]-e[2 i, 2 j-1,2 i-1,2 j]
\end{array}\right)^{\sigma} \\
& +\frac{1}{4 n(n+1)} \sum_{\sigma \in S} \sum_{j, k \neq i}\left(\begin{array}{c}
e[2 j-1,2 k, 2 j, 2 k-1]+e[2 j, 2 k-1,2 j-1,2 k] \\
-e[2 j-1,2 k-1,2 j, 2 k]-e[2 j, 2 k, 2 j-1,2 k-1]
\end{array}\right)^{\sigma} \\
& +\frac{n-1}{2 n(n+1)}\left(\begin{array}{c}
2 e[2 i-1,2 i-1,2 i, 2 i]+2 e[2 i, 2 i, 2 i-1,2 i-1]-e[2 i-1,2 i, 2 i-1,2 i] \\
-e[2 i-1,2 i, 2 i-1]-e[2 i, 2 i-1,2 i-1,2 i]-e[2 i-1,2 i, 2 i, 2 i-1]
\end{array}\right) \\
& +\frac{1}{4(n+1)} \sum_{\sigma \in S} \sum_{j=1}^{n}\left(\begin{array}{c}
e[2 i-1,2 j-1,2 i, 2 j]+e[2 i, 2 j-1,2 i-1,2 j] \\
-e[2 i-1,2 j, 2 i, 2 j-1]-e[2 i, 2 j, 2 i-1,2 j-1]
\end{array}\right)^{\sigma} \\
& +\frac{2 n-1}{2(2 n+1)(2 n+2)} \sum_{\sigma \in S} \sum_{j \neq i}\left(\begin{array}{c}
e[2 i-1,2 j, 2 j-1,2 i]+e[2 i, 2 j-1,2 j, 2 i-1] \\
-e[2 i-1,2 j-1,2 j, 2 i]-e[2 i, 2 j, 2 j-1,2 i-1]
\end{array}\right)^{\sigma} \\
& +\frac{1}{2(2 n+1)(2 n+2)} \sum_{\sigma \in S} \sum_{j, k \neq i}\left(\begin{array}{c}
e[2 j-1,2 k-1,2 j, 2 k]+e[2 j, 2 k, 2 j-1,2 k-1] \\
-e[2 j-1,2 k, 2 j, 2 k-1]-e[2 j, 2 k-1,2 j-1,2 k]
\end{array}\right)^{\sigma} \\
& +\frac{(2 n-1)(2 n-2)}{6(2 n+1)(2 n+2)}\left(\begin{array}{c}
2 e[2 i-1,2 i-1,2 i, 2 i]+2 e[2 i, 2 i, 2 i-1,2 i-1]-e[2 i-1,2 i, 2 i-1,2 i] \\
-e[2 i, 2 i-1,2 i, 2 i-1]-e[2 i, 2 i-1,2 i-1,2 i]-e[2 i-1,2 i, 2 i, 2 i-1]
\end{array}\right) \\
& +\frac{n-1}{2(n+1)}(e[2 i-1,2 i-1,2 i, 2 i]-e[2 i, 2 i, 2 i-1,2 i-1]) \\
& +\frac{1}{\sigma(n+1)} \sum_{\sigma \in S} \sum_{j \neq i}\left(2 i-e\left[\begin{array}{c}
e[2 i-1,2 j, 2 j-1,2 i]+e[2 i, 2 j, 2 j-1,2 i-1] \\
-e[2 i-1,2 j-1,2 j, 2 i]-e[2 i, 2 j-1,2 j, 2 i-1]
\end{array}\right)^{\sigma}\right. \\
& +1,2 i-1,2 i, 2 i]^{\sigma}
\end{aligned}
$$


with the parts of this expansion respectively in $V_{2 n+1}, V_{n+1}, V_{n}, V_{0}, V_{-1}$, and $V_{-3}$. In these expansions, $S=(\mathbb{Z} / 2 \mathbb{Z})^{2}$ denotes the group of permutations $\{$ id, $(1,2),(3,4),(1,2)(3,4)\}$.

The expansion in eigenvectors of $e[2 i, 2 i, 2 j, 2 j]$ is

$$
\frac{1}{6}\left(\begin{array}{c}
2 e[2 i, 2 i, 2 j, 2 j]+2 e[2 j, 2 j, 2 i, 2 i]-e[2 i, 2 j, 2 i, 2 j] \\
-e[2 j, 2 i, 2 j, 2 i]-e[2 i, 2 j, 2 j, 2 i]-e[2 j, 2 i, 2 i, 2 j]
\end{array}\right)+\frac{1}{2}\left(\begin{array}{c}
e[2 i, 2 i, 2 j, 2 j] \\
-e[2 j, 2 j, 2 i, 2 i]
\end{array}\right)+\frac{1}{6} \sum_{\sigma \in \mathfrak{S}_{4}}^{\prime} e[2 i, 2 i, 2 j, 2 j]^{\sigma}
$$

with each part respectively in $V_{0}, V_{-1}$ and $V_{-3}$; and similarly for the expansions of $e[2 i-1,2 i-1,2 j, 2 j]$ or $e[2 i-1,2 i-1,2 j-1,2 j-1]$. Finally, we skip the expansion in eigenvectors of $e[2 i-1,2 i, 2 j-1,2 j]$, since it is two pages long.

Acknowledgements Many thanks are due to Yacine Barhoumi, Philippe Biane, Florent BenaychGeorges, Paul Bourgade, Reda Chhaibi, Djalil Chafaï, Kenneth Maples, Ashkan Nikeghbali and Simon Pépin-Lehalleur for discussions around the cut-off phenomenon and the theory of Lie groups. The author would also like to thank the anonymous referee, whose comments allowed in particular to precise Theorem 7 .

\section{References}

1. Aldous, D., Diaconis, P.: Shuffling cards and stopping times. Amer. Math. Monthly 93(5), 333348 (1986)

2. Applebaum, D.: Infinitely divisible central probability measures on compact Lie groups Regularity, semigroups and transition kernels. Ann. Probab. 39(6), 2474-2496 (2011)

3. Bröcker, T., Dieck, T.: Representations of Compact Lie Groups, vol. 98 of Graduate Texts in Mathematics. Springer-Verlag (1985)

4. Bayer, D., Diaconis, P.: Trailing the dovetail shuffle to its lair. Ann. Appl. Prob. 2(2), 294-313 (1992)

5. Chen, G.-Y., Saloff-Coste, L.: The cutoff phenomenon for ergodic Markov processes. Electron. J. Probab. 13(3), 26-78 (2008)

6. Ceccherini-Silberstein, T., Scarabotti, F., Tolli, F.: Harmonic Analysis on Finite Groups, vol. 108 of Cambridge Studies in Advanced Mathematics. Cambridge University Press (2008)

7. Diaconis, P.: The cutoff phenomenon in finite Markov chains. Proc. Nat. Acad. Sci. USA 93(4), 1659-1664 (1996)

8. Diaconis, P., Shahshahani, M.: On the eigenvalues of random matrices. J. Appl. Probab. 31, 49-62 (1994)

9. Diaconis, P., Saloff-Coste, L.: Random walks on finite groups: a survey of analytic techniques. In: Heyer, H. (ed.) Prob. Meas. on Groups XI, pp. 44-75. World Scientific Singapore (1996)

10. Faraut, J.: Analysis on Lie Groups: an Introduction, vol. 110 of Cambridge Studies in Advanced Mathematics. Cambridge University Press (2008)

11. Fulton, W., Harris, J.: Representation Theory, vol. 129 of Graduate Texts in Mathematics. Springer-Verlag (1991)

12. Gasper, G.: Linearization of the product of Jacobi polynomials. I and II. Can. J. Math. 22, 171$175,582-593(1970)$

13. Goodman, R., Wallach, N.R.: Symmetry, Representations, and Invariants, vol. 255 of Graduate Texts in Mathematics. Springer-Verlag (2009)

14. Helgason, S.: Differential Geometry, Lie Groups, and Symmetric Spaces. Academic Press (1978)

15. Helgason, S.: Groups and Geometric Analysis. Integral Geometry, Invariant Differential Operators, and Spherical Functions. Academic Press (1984)

16. Heckman, G., Schlichtkrull, H.: Harmonic Analysis and Special Functions on Symmetric Spaces, vol. 16 of Perspectives in Mathematics. Academic Press (1994)

17. Hunt, G.A.: Semigroups of measures on Lie groups. Trans. Am. Math. 81, 264-293 (1956)

18. Lévy, T.: Asymptotics of Brownian motions on classical Lie groups, the master field on the plane, and the Makeenko-Migdal equations. arXiv:1112.2452v1 [math-ph] (2011)

19. Liao, M.: Lévy processes and Fourier analysis on compact Lie groups. Ann. Probab. 32(2), 553$1573(2004)$ 
20. Liao, M.: Lévy processes in Lie groups, vol. 162 of Cambridge Tracts in Mathematics. Cambridge University Press (2004)

21. Macdonald, I.G.: Symmetric Functions and Hall Polynomials, 2nd edn. Oxford Mathematical Monographs. Oxford University Press (1995)

22. Porod, U.: The cut-off phenomenon for random reflections. Ann. Probab. 24(1), 74-96 (1996)

23. Porod, U.: The cut-off phenomenon for random reflections II: complex and quaternionic cases. Probab. Theory Related Fields 104(2), 181-209 (1996)

24. Rosenthal, J.S.: Random rotations: characters and random walks on $\mathrm{SO}(N)$. Ann. Probab. 22, 398-423 (1994)

25. Saloff-Coste, L.: Precise estimates on the rate at which certain diffusions tend to equilibrium. Math. Z. 217, 641-677 (1994)

26. Saloff-Coste, L.: Lectures on finite Markov chains. In: Lectures on probability theory and statistics: École d'été de Probabilités de Saint-Flour XXVI-1996. Lecture Notes in Mathematics, vol. 1665, pp. 301-413 (1997)

27. Saloff-Coste, L.: On the convergence to equilibrium of Brownian motion on compact simple Lie groups. J. Geom. Anal. 14(4), 715-733 (2004)

28. Saloff-Coste, L.: Analysis on compact Lie groups of large dimension and on connected compact groups. Colloq. Math. 118(1), 183-199 (2010)

29. Varadarajan, V.S.: An Introduction to Harmonic Analysis on Semisimple Lie Groups, vol. 16 of Cambridge Studies in Advanced Mathematics. Cambridge University Press (1989) 